\title{
ASYMPTOTIC EXPANSIONS OF EXPONENTIAL INTEGRALS AND NEWTON DIAGRAMS *
}

\author{
STÉPHANE ROSSIGNOL ${ }^{\dagger}$
}

\begin{abstract}
We study the asymptotic expansion, as $\lambda \rightarrow 0^{+}$, of integrals of the form $J_{H, \chi}(\lambda)=$ $\int \exp (H(x) / \lambda) \cdot \chi(x) d x$, where $H$ and $\chi$ are smooth from $\mathbb{R}^{p}$ to $\mathbb{R}, H$ has a unique (degenerate) maximum at $0, \chi$ has compact support a neighborhood of 0 .

If $p=2$ or if the Newton Diagram of $H$ contains only one facet, we give an algorithm to compute explicitely the complete asymptotic expansion of $J_{H, \chi}(\lambda)$. In the general case, we show how to write $J_{H, \chi}(\lambda)$ as a linear combination of simpler integrals, involving only the fundamental part of $H$. We give an equivalent of the first term of the expansion of $J_{H, \chi}(\lambda)$, and specify the exact form of this first term under a simple additional condition.
\end{abstract}

1. Introduction. Computations involving integrals of the type

$$
J_{H, \chi}(\lambda)=\int \exp (H(x) / \lambda) \cdot \chi(x) d x
$$

as a small parameter $\lambda$ tends to $0^{+}$, appear very often in analysis and in probability. For $\chi$ and $H$ smooth, let us recall that Laplace's method shows that for the asymptotic expansion of $J(\lambda)$, the major contribution comes from neighborhood of points where $H$ attains its absolute maximum. We will assume in this article, that there is a unique such point, which will be 0 to simplify, with $H(0)=0$. We have then henceforth, $H(x) \leq H(0)=0$ for all $x \in \mathbb{R}^{p}, H$ of absolute isolated maximum at $0 . \chi$ and $H$ will be in $C^{\infty}\left(\mathbb{R}^{p}, \mathbb{R}\right)$, and $\chi$ with compact support a neighborhood of 0 . $H^{\prime}(0)=0$ and $H^{\prime \prime}(0) \leq 0$.

$H$ "(0) negative definite is the so-called nondegenerate case. Under this hypothesis, Morse's lemma is valid, the asymptotic expansion is known and easily computable (see Combet [3] p.6, or Wong [14] p.495).

If the rank of $H^{\prime \prime}(0)$ is between 1 and $p-1$, one can dissociate a degenerate part and a nondegenerate part (see Tougeron's lemma, in Combet [3] p.30-34). That's why we will only study the degenerate case with $H^{\prime \prime}(0)=0$.

In Arnold-Varchenko [2] and Varchenko [12], is shown the existence of a complete asymptotic expansion in powers of $\lambda$ and $\ln \lambda$. It is based on toric resolutions of singularities, but for analytic $H$ only, and especially the proof is not constructive. The computation of the coefficients then is very difficult. To compute them explicitly, it seems necessary to avoid desingularisation. It is what Vasil'ev does, he gives the first term, for analytic functions. Dostal and Gaveau [5] give the first term for a polynomial function $H$ with negative coefficients, when $\chi=1$, under the hypothesis that at most two faces of the Newton polyhedron intersect the axes bissectrice. Kaminski and Paris [8], using Melling-Barnes integrals, give the expansion for a class of functions if $p=2$ or $p=3$. Denef and Sargos [4] obtain information related to the Newton Polyhedron on the poles of $\int f_{+}^{s}(x) \phi(x) d x$ where $f_{+}^{s}=\max \left(f^{s}, 0\right)$, using change of variables and dissections in $\mathbb{R}^{p}$.

We provide an algorithm to compute the complete sequence of the coefficients of the expansion when $p=2$ or when the Newton diagram of $H$ has only one facet (Theorem 3). Otherwise, Theorem 1 shows that the asymptotic expansion of $J_{H, \chi}(\lambda)$

\footnotetext{
*Received February 11, 2000; accepted for publication October 15, 2003.

†Université de Versailles-Saint-Quentin, département de mathématiques, 45 avenue des USA, 78035 Versailles Cedex, France (rossignol@math.uvsq.fr).
} 
can be obtained from that of simpler integrals involving only the fundamental part of $H$. Theorem 2 gives an equivalent of the first term. The coefficient of this first term is computed under a simple additional condition.

We will assume that $\chi$ and $H$ are smooth functions from $\mathbb{R}^{p}$ to $\mathbb{R}$, with $\chi$ of compact support a neighborhood $K$ of 0 , and $H$ of unique isolated absolute maximum at 0 . The other hypotheses will be relevant to the Newton diagram of $H$ at 0 .

Let us recall that if $H(x) \sim \sum_{\mu \in \mathbb{N}^{p}} a_{\mu} x^{\mu}$ as $x \rightarrow 0$ is the Taylor series of $H$ at 0 , its Newton polyhedron $\Pi(H)$ is the convex hull of $\left\{\mu \in \mathbb{N}^{p} \backslash\{0\} ; a_{\mu} \neq 0\right\}+\mathbb{R}_{+}^{p}$, its Newton diagram $\Delta(H)$ is the union of the compact faces of $\Pi(H)$.

We show (see Theorem 3) that if $H$ is convenient, of nondegenerate principal part (in a meaning precise later), then $H$ has the asymptotic expansion, as $\lambda \rightarrow 0^{+}$

$$
J_{H, \chi}(\lambda) \sim \sum_{k} \sum_{l=0}^{N(k)} c_{k, l} \lambda^{k} .(\ln \lambda)^{l}
$$

where we explain how to compute explicitly the coefficients $c_{k, l}$ if $p=2$ or if $\Delta(H)$ contains only one facet. The coefficients $c_{k, l}$ will be functions of $\chi$ and $H$, and especially of the Newton polyhedron of the phase $H$ ( $l$ is here an integer, and the real $k$ form an unbounded increasing sequence). They can be computed by applying successively Propositions 1 to 7. These propositions provide an algorithm to evaluate the coefficients.

This algorithm is a multistep process of simplification of the integral $J_{H, \chi}(\lambda)$ when $H$ and $\chi$ satisfy the Hypothesis H1. It contains 2 main parts:

- First, Propositions 1 to 5 reduce the problem to the study of $L(\theta, \gamma, P)=$ $\int_{\mathbb{R}_{+}^{p}} x^{\theta-1}(\ln x)^{\gamma} \cdot \exp \left(\frac{P(x)}{\lambda}\right) d x$ where $P$ is a polynomial of Newton Diagram $\Delta(H)$ (more precisely under Hypothesis HP). These 5 propositions are used to prove Theorem 1.

- Second, Propositions 6 and 7 give the computation of the asymptotic expansion of $L(\theta, \gamma, P)$, when $(\theta, \gamma, P)$ satisfies Hypothesis HP, if $p=2$ or if the Newton diagram has only one facet.

In Proposition 1 (truncation), the integral is replaced by one taken over a small ball $B(\delta)$ centered at the origin. In Proposition 2 (approximation of the amplitude) is shown that the amplitude $\chi$ can be replaced by the first terms of its Taylor series at 0 . Proposition 3 (reduction of the phase) replaces the phase function $H$ by its principal part $\hat{H}$, i.e. the terms of its Taylor series coming from its Newton diagram. Proposition 4 (extension of the domain) simply removes the bound $B(\delta)$.

Proposition 5 allows to simplify $\hat{H}$ by removing the part of $\hat{H}$ coming from a facet of $\Delta$ (except for the vertices). Applying Proposition 5 several times leads to integrals with phases arising from vertices and individual facets of $\Delta$.

Proposition 6 allows to remove one vertex of the Newton diagram $\Delta$ of $P$ (when $p=2$ ). More precisely, $L(\theta, \gamma, P)$ is written as a linear combination of integrals of the same type, but with Newton diagram $\Delta^{*}$ having one vertex (and one facet) less than $\Delta$. This procedure can be reiterated, to remove progressively the vertices, until having integrals of the type $L(\theta, \gamma, P)$ with $P$ of Newton diagram with only one facet.

This easy case is solved by Proposition 7 (for any $p \geq 2$ ).

This paper is organized as follows. In Section 2 we give the most important definitions, we state the theorems and detail in Propositions 1 to 8 the algorithm of simplification of the integral and of computation of the coefficients of the asymptotic expansion. The proofs of Propositions 1 to 5 are given in Section 3. Proposition 6 
is proven in Section 4 and Proposition 7 in Section 5. In Section 6 we develop an example of calculus to make easier the understanding of the algorithm.

2. Statement of the results. In this section we first introduce the most important definitions. After stating our theorems, we give an algorithm of simplification of $J_{H, \chi}(\lambda)$, then explain how it is possible to remove progressively the vertices of the Newton diagram (for $p=2$ ). Finally, we show how to compute the complete asymptotic expansion when the Newton diagram has a unique facet (for any $p$ ).

2.1. Definitions and notations. Henceforth $H$ is a smooth function on $\mathbb{R}^{p}$, with $H(0)=0$, and $H(x) \leq 0$ for any $x \in \mathbb{R}^{p}$. We recall first a few definitions.

2.1.1. Newton polyhedron and Newton diagram. For $x=\left(x_{1}, \ldots, x_{p}\right) \in$ $\mathbb{R}^{p}, \mu=\left(\mu_{1}, \ldots, \mu_{p}\right) \in \mathbb{N}^{p}$, we set $x^{\mu}=\prod_{i=1}^{p} x_{i}^{\mu_{i}}$.

Let $B \subseteq \mathbb{N}^{p} \backslash\{0\}$.

- The Newton polyhedron of $B$ is $\Pi(B)=\operatorname{Conv}\left(B+\mathbb{R}_{+}^{p}\right)$. It's a convex polyhedron.

- The Newton diagram $\Delta(B)$ of $B$ is the union of the compact faces of $\Pi(B)$.

- $\Delta$ is said to be a Newton diagram if there exists $B \subseteq \mathbb{N}^{p} \backslash\{0\}$ such that $\Delta=\Delta(B)$.

- Let us put then $S_{\Delta}=\left\{\mu \in \mathbb{N}^{p} ; \mu\right.$ vertex of $\left.\Delta\right\}$ (often denoted simply by $S$ )

- The cone $\mathcal{C}(B)$ generated by a part $B$ of $\mathbb{R}_{+}^{p}$ is the set of linear combinations of elements of $B$, with non negative coefficients. The interior of $\mathcal{C}(B)$ is denoted by $\operatorname{int} \mathcal{C}(B)$.

- A face $F$ of $\Delta$ is the intersection of $\Delta$ with any supporting hyperplane.

If $l(\mu)=\sum d_{i} \mu_{i}=1$ is the equation of such an hyperplane, then for any $\mu \in \Pi$,

$l(\mu)=1 \Leftrightarrow \mu \in F$ and $l(\mu)>1 \Leftrightarrow \mu \in \Pi \backslash F$

- A facet is a face of codimension 1 .

2.1.2. Newton diagram of a function, convenient functions. Recall $H \in$ $C^{\infty}\left(\mathbb{R}^{p}, \mathbb{R}\right)$, with $H(0)=0$, and consider its Taylor series at the origin $H(x) \sim$ $\sum_{\mu \in \mathbb{N}^{p}} a_{\mu} x^{\mu}$.

- The support of $H$ is $\Sigma(H)=\left\{\mu \in \mathbb{N}^{p} ; a_{\mu} \neq 0\right\}$.

- The Newton polyhedron $\Pi(H)$ and the Newton diagram $\Delta(H)$ of $H$ are the Newton polyhedron and the Newton diagram of the support of $H$ i.e. $\Pi(H)=\Pi(\Sigma(H))$ and $\Delta(H)=\Delta(\Sigma(H))$.

- $H$ is called convenient (or suitable) if $\Delta(H)$ intersects each coordinate axis.

- If $H$ is analytic and have an isolated maximal point at 0 , then $H$ is always convenient (see Vasil'ev [13] Prop 1.2). If $H$ is non analytic, a counterexample is $H(x, y)=$ $-x^{2}\left(1+\exp \left(-1 / y^{2}\right)\right)$ smooth but not convenient.

- The polynomial $\hat{H}(x)=\sum_{\mu \in \Delta} a_{\mu} x^{\mu}$ is called the principal part of the expansion of $H$ at 0 , where $\Delta=\Delta(H)$.

- For any subset $B$ of $\Delta$, we set $H_{B}(x)=\sum_{\mu \in B} a_{\mu} x^{\mu}$

2.1.3. Index of a point. Let $\Delta=\Delta(B)$ be a Newton diagram. For any facet $F$ of $\Delta$, let us define $A_{F}(\mu)=\sum_{i=1}^{p} d_{i} \mu_{i}$, where $\sum_{i=1}^{p} d_{i} \mu_{i}=1$ is the equation of the hyperplane including $F$. The map $\mu \mapsto A_{F}(\mu)$ is a linear form.

We set $A_{\Delta}(\mu)=\inf \left(A_{F}(\mu) ; F\right.$ facet of $\left.\Delta\right)$. We will often write $A(\mu)$ for $A_{\Delta}(\mu)$ where $\Delta=\Delta(H)$.

One can easily show that $A_{\Delta}\left(\mu+\mu^{\prime}\right) \geq A_{\Delta}(\mu)+A_{\Delta}\left(\mu^{\prime}\right)$ and that $A_{\Delta}(t \mu)=t A_{\Delta}(\mu)$ for $t \in \mathbb{R}_{+}$

Definition. $A_{F}(\mu)$ is called the index of $\mu$ with respect to the facet $F$. Similarly $A_{\Delta}(\mu)$ is called the index of $\mu$ with respect to the Newton Diagram $\Delta$. Note that for $\mu=(1 ; \ldots ; 1), A_{\Delta}(\mu)$ is the index $\beta$ of the Newton diagram $\Delta$ (see Arnold [1] p.22-23). 
Index of a function. If $\chi$ is a smooth function on $\mathbb{R}^{p}$, and $\Delta$ a Newton diagram, we define the index of $\chi$ with respect to $\Delta$ by

$$
A_{\Delta}(\chi)=\inf \left\{A_{\Delta}(\mu) ; q_{\mu-1} \neq 0\right\}
$$

where $\chi(x) \sim \sum_{\mu \in \mathbb{N}^{p}} q_{\mu} x^{\mu}$ is the Taylor series of $\chi$ at 0 .

If $\chi(0) \neq 0$, it is clear that $A_{\Delta}(\chi)=A_{\Delta}(1)$

Remarks. If $\mu \in \mathcal{C}(F), A_{F}(\mu)=\frac{d(O, \mu)}{d(O, N)}$ where $\{N\}=(0, \mu) \cap F$, and if $\mu \in \mathcal{C}(\Delta), A_{\Delta}(\mu)=\frac{d(O, \mu)}{d(O, P)}$ where $\{P\}=(0, \mu) \cap \Delta$

$d$ is here any distance.

$A_{\Delta}(\mu)=1 \Leftrightarrow \mu \in \Delta$

$A_{\Delta}(\mu) \geq 1 \Leftrightarrow \mu$ is "above" $\Delta$.

2.1.4. Nondegeneracy. (i) The principal part $\hat{H}$ of $H$ is said to be $\mathbb{R}$ nondegenerate if $\nabla H_{F} \neq 0$ on $\left(\mathbb{R}^{*}\right)^{p}$, for every face $F$ of $\Delta$ (see Arnold [2], Kouchnirenko [9]).

(ii) $\hat{H}$ is said to be extremally nondegenerate (in Vasil'ev [13] meaning), if there exists $c>0$, such that, for $x$ in a neighborhood of 0 :

$$
\hat{H}(x) \leq-c . \sum_{\mu \in S_{\Delta}} x^{\mu}
$$

For every smooth function $H$ on $\mathbb{R}^{p}$, with $H(0)=0$ and $H(x) \leq 0$ for every $x \in \mathbb{R}^{p}$, the next lemmas are valid:

Lemma 1. For every face $F$ of $\Delta, H_{F}(x) \leq 0, \forall x \in \mathbb{R}^{p}$.

Lemma 2. Every vertex $\mu^{\prime}$ of $\Delta$ has even coordinates, and $a_{\mu^{\prime}}<0$.

The next lemma gives 4 equivalent characterizations of the nondegeneracy.

Lemma 3. For $\hat{H}$ principal part of $H$, let :

(iii) For any face $F, H_{F}<0$ on $\left(\mathbb{R}^{*}\right)^{p}$

(iv) For any face $F, H_{F}<0$ on $\left(\mathbb{R}^{*}\right)^{p}$ and $\hat{H}<0$ on $\left(\mathbb{R}^{*}\right)^{p}$

Then $(i),($ ii), $($ iii $)$ and $(i v)$ are equivalent.

According to Lemma 3, the polynomial $\hat{H}$ principal part of $H$ will be said to be non degenerate if one of the equivalent conditions $(i),(i i),(i i i),(i v)$ is true.

2.1.5. First term of the expansion of $L(\theta, \gamma, P)$. For a polynomial $P$, we give in Lemma 4 an estimation of the first term of

$$
L(\theta, \gamma, P)=\int_{\mathbb{R}_{+}^{p}} x^{\theta-1} \cdot(\ln x)^{\gamma} \cdot \exp \left(\frac{P(x)}{\lambda}\right) d x
$$

which will be useful subsequently.

Hypothesis HP. We say that $(\theta, \gamma, P)$ satisfies the HP hypothesis if the following assumptions hold:

- $P$ is a polynomial which is its own principal part, i.e. denoting by $\Delta$ the Newton diagram of $P$, we can write $P$ as $P(x)=\hat{P}(x)=\sum_{\mu \in \Delta} a_{\mu} x^{\mu}$. Moreover we assume that $P$ is non degenerate, $P(x) \leq 0, P(0)=0$, but 0 is not necessarily an isolated maximum of $P$, and $P$ may not be convenient. 
- $\theta \in] 0 ;+\infty\left[{ }^{p}\right.$, with $\theta \in \operatorname{int} \mathcal{C}(\Delta)$ and $\gamma \in \mathbb{N}^{p}$.

Lemma 4. Let $L_{B}(\theta, \gamma, P)=\int_{B} x^{\theta-1} \cdot(\ln x)^{\gamma} \cdot \exp \left(\frac{P(x)}{\lambda}\right) d x$, for any Borel set $B$ of $\mathbb{R}_{+}^{p}$, where $(\theta, \gamma, P)$ satisfies the HP hypothesis. Then

(i) $L_{B}(\theta, \gamma, P)$ is absolutely convergent.

(ii) For any $\epsilon>0$,

$$
L_{B}(\theta, \gamma, P)=o\left(\lambda^{A_{\Delta}(\theta)-\epsilon}\right) \quad \text { as } \lambda \rightarrow 0^{+}
$$

In particular

$$
L(\theta, \gamma, P)=o\left(\lambda^{M}\right)
$$

as soon as $A_{\Delta}(\theta)>M$

(iii) If in addition $\theta$ is in the interior of the cone generated by a facet $F$ of $\Delta$, then as $\lambda \rightarrow 0^{+}$

$$
L_{B}(\theta, \gamma, P)=C_{\gamma}(F) \cdot \lambda^{A_{\Delta}(\theta)} \cdot(\ln \lambda)^{\gamma_{1}+. .+\gamma_{p}} \int_{B} x^{\theta-1} \exp \left(P_{F}(x)\right) d x .(1+o(1))
$$

with $C_{\gamma}(F)=\prod_{i=1}^{p}\left(A_{F}\left(e_{i}\right)\right)^{\gamma_{i}}$ and $e_{1}=(1 ; 0 . . ; 0), . ., e_{p}=(0 ; . . ; 0 ; 1)$

Remarks. We are mainly interested by $L(\theta, \gamma, P)=L_{B}(\theta, \gamma, P)$ for $B=\mathbb{R}_{+}^{p}$.

(i) and (ii) remain true for the integral

$L^{\prime}(\theta, \gamma, P)=\int_{\mathbb{R}_{+}^{p}} x^{\theta-1} \cdot|(\ln x)|^{\gamma} \cdot \exp \left(\frac{P(x)}{\lambda}\right) d x$ and on the other octans, i.e. for $L^{\prime \prime}(\theta, \gamma, P)=\int_{\mathbb{R}^{p}}|x|^{\theta-1} \cdot|(\ln |x|)|^{\gamma} \cdot \exp \left(\frac{P(x)}{\lambda}\right) d x$

Index of an integral. We call index of the integral $L(\theta, \gamma, P)$ the index $A_{\Delta}(\theta)$ of $\theta$ with respect to the Newton Diagram $\Delta$. It gives a useful order of magnitude of $L(\theta, \gamma, P)$ as $\lambda \rightarrow 0^{+}$.

Similarly, $A_{\Delta}(\theta)$ is the index of $L^{\prime}(\theta, \gamma, P)$ and $L "(\theta, \gamma, P)$

2.2. The theorems. We will now state our main results. Let us first give the needed hypothesis on $H$ and $\chi$.

Hypothesis H1. We say that $\chi$ and $H$ satisfy the $\mathrm{H} 1$ hypothesis if the following assumptions are valid:

- $\chi \in C^{\infty}\left(\mathbb{R}^{p}, \mathbb{R}\right)$, with support $K$ a compact neighborhood of 0

- $H \in C^{\infty}\left(\mathbb{R}^{p}, \mathbb{R}\right)$, of unique isolated maximal point at 0 , with $H(0)=0$. Moreover, $H$ is convenient, of non degenerate principal part $\hat{H}$.

We will denote by $\sum_{\mu \in \mathbb{N}^{p}} q_{\mu} x^{\mu}$ and $\sum_{\mu \in \mathbb{N}^{p}} a_{\mu} x^{\mu}$ the Taylor series at 0 of $\chi$ and $H$ respectively.

Theorem 1 shows that $J_{H, \chi}(\lambda)$ has the same asymptotic expansion that a linear combination of simpler integrals, involving only monomials coming from the vertices and one facet of the Newton diagram of $H$.

ThEOREM 1. Let $\chi$ and $H$ satisfy $H 1$. Then for any $M \geq 0$, there exist constants $d_{k, \theta}$ and $d_{k, \theta, F}$ (only finitely many different from 0), where $k \in \mathbb{N}, \theta \in \mathbb{N}^{* p}, F$ belongs to the set of facets of $\Delta$, such that as $\lambda \rightarrow 0^{+}$

$$
\begin{aligned}
& \text { (a) } J_{H, \chi}(\lambda)=\sum_{k} \lambda^{-k} \sum_{\theta} d_{k, \theta} \int_{\mathbb{R}^{p}} x^{\theta-1} \exp \left(\frac{\hat{H}(x)}{\lambda}\right) d x+o\left(\lambda^{M}\right) \\
& \text { (b) } J_{H, \chi}(\lambda)=\sum_{k} \lambda^{-k} \sum_{\theta, F} d_{k, \theta, F} \int_{\mathbb{R}^{p}} x^{\theta-1} \exp \left(\frac{H_{S \cup F}(x)}{\lambda}\right) d x+o\left(\lambda^{M}\right)
\end{aligned}
$$


Here $\Delta$ is the Newton diagram of $H, H_{S \cup F}(x)=\sum_{\mu \in S \cup F} a_{\mu} x^{\mu}$ and $\hat{H}(x)=$ $\sum_{\mu \in \Delta} a_{\mu} x^{\mu}$

Proof of Theorem 1. (a) comes from Propositions 1 to 4 applied successively. Then, the repeated application of Proposition 5 leads to (b).

Theorem 2 gives an equivalent of the first term of $J_{H, \chi}(\lambda)$, and specifies the exact form of this first term under a simple additional condition.

Put $E=\left\{\theta \in \mathbb{N}^{p} ; q_{\theta-1} \neq 0\right.$ and $\left.A_{\Delta}(\theta)=A_{\Delta}(\chi)\right\} . E$ is the set of monomials of the Taylor series of $\chi$ contributing to the leading term of the asymptotic expansion of $J_{H, \chi}(\lambda)$. It is the part of the Taylor series of $\chi$ of minimal indices with respect to $\Delta$. $E$ is always finite.

Subsequently, we denote $(0 ; \ldots ; 0) \in \mathbb{N}^{p}$ by 0 and $(1 ; \ldots ; 1)$ by 1 .

THEOREM 2. If $\chi$ and $H$ satisfy $H 1$, the first term of the expansion of $J_{H, \chi}(\lambda)$ as $\lambda \rightarrow 0^{+}$can be computed with

$$
\text { (a) } J_{H, \chi}(\lambda)=\sum_{\theta \in E} q_{\theta-1} \int_{\mathbb{R}^{p}} x^{\theta-1} \exp \left(\frac{\hat{H}(x)}{\lambda}\right) d x+o\left(\lambda^{A_{\Delta}(\chi)}\right)
$$

Moreover, if for any $\theta \in E$, the line $(0 ; \theta)$ intersects only one facet of $\Delta$, then

$$
\text { (b) } J_{H, \chi}(\lambda)=\lambda^{A_{\Delta}(\chi)} \sum_{\theta \in E} q_{\theta-1} \int_{\mathbb{R}^{p}} x^{\theta-1} \exp \left(H_{F}(x)\right) d x+o\left(\lambda^{A_{\Delta}(\chi)}\right)
$$

Remark. If $\chi(0) \neq 0$, then $E=\{1\}, A_{\Delta}(\chi)=A_{\Delta}(1)$ and Theorem 2 becomes

$$
\text { (a) } J_{H, \chi}(\lambda)=q_{0} \int_{\mathbb{R}^{p}} \exp \left(\frac{\hat{H}(x)}{\lambda}\right) d x+o\left(\lambda^{A_{\Delta}(1)}\right)
$$

If the line $(0 ; 1)$ intersects only one facet $F$ of $\Delta$, then

$$
\text { (b) } J_{H, \chi}(\lambda)=\lambda^{A_{\Delta}(1)} q_{0} \int_{\mathbb{R}^{p}} \exp \left(H_{F}(x)\right) d x+o\left(\lambda^{A_{\Delta}(1)}\right)
$$

Proof of Theorem 2. (a) is obtained with Propositions 1 to 4 , taking $M=A_{\Delta}(\chi)$ in Proposition 2 and Proposition 3.

(b) is due to Lemma 4 (iii) applied on (a).

In the bidimensional case (or in the rather simple case of a Newton diagram limited to a unique facet), the complete asymptotic expansion is given by Theorem 3 .

TheOREM 3. Let $J_{H, \chi}(\lambda)=\int \exp (H(x) / \lambda) \cdot \chi(x) d x$ where $\chi$ and $H$ satisfy $H 1$ hypothesis. If $p=2$ or if $\Delta$ contains only one facet, then $J_{H, \chi}(\lambda)$ has the asymptotic expansion :

$$
J_{H, \chi}(\lambda) \sim \sum_{k} \sum_{l=0}^{N(k)} c_{k, l} \lambda^{k} \cdot(\ln \lambda)^{l} \quad \text { as } \lambda \rightarrow 0^{+}
$$

The $c_{k, l}$ are computed by applying successively to $J_{H, \chi}(\lambda)$ Propositions 1 to $\%$. $k$ here belongs to an increasing and unbounded sequence of real numbers, $l \in \mathbb{N}$. 
Proof of Theorem 3. If $\Delta$ contains only one facet, Theorem 3 comes staightfully from Proposition 7 applied to Theorem 1 (a).

If $\Delta$ contains several facets but $p=2$, the repeated application of Proposition 6 to Theorem 1 (b) will allow to remove vertices of $\Delta$, leading to the same type of integral with a unique facet. The use of Proposition 7 is then sufficient to end the proof.

Note that the existence of this expansion was known for $\chi$ and $H$ analytic (see Combet [3], or Arnold-Varchenko [2], Varchenko [12]), but without real method to compute the coefficients.

2.3. Algorithm of simplification of $J_{H, \chi}(\lambda)$. The proof of Theorem 1 is obtained by applying successively Propositions 1 to 5 . These 5 propositions form an algorithm of simplification of our integral $J_{H, \chi}(\lambda)$.

For a better understanding of this algorithm, we recommend to follow the detailed example in Section 6, while reading the propositions.

Subsequently, $H$ and $\chi$ are assumed to satisfy Hypothesis H1. We put $B(\delta)=\left\{x \in \mathbb{R}^{p} ;\|x\|<\delta\right\}$ for any $\delta>0$.

2.3.1. Truncation. Let us first show that, as usual with Laplace's method, in $\int \exp (H(x) / \lambda) \cdot \chi(x) d x$ the major contribution comes from a neighborhood of 0 , the point where $H$ attains its greatest value:

Proposition 1. For any $\delta>0$ such that the ball $B(\delta)$ is included in the support $K$ of $\chi$, there exists $d>0$ such that, as $\lambda \rightarrow 0^{+}$

$$
\int \chi(x) \cdot \exp (H(x) / \lambda) d x=\int_{B(\delta)} \chi(x) \cdot \exp (H(x) / \lambda) d x+O\left(e^{-d / \lambda}\right)
$$

2.3.2. Approximation of the amplitude. It is shown that the asymptotic expansion of $J_{H, \chi}(\lambda)$ to order $M$, is not modified if the beginning of the Taylor series of $\chi$ at 0 is put instead of $\chi(x)$ : at 0 .

Proposition 2. Let $\sum_{\theta \in \mathbb{N}^{p}} q_{\theta} x^{\theta}=\sum_{\theta \in \mathbb{N}^{* p}} q_{\theta-1} x^{\theta-1}$ be the Taylor series of $\chi$ Then for each $M \in \mathbb{R}_{+}$, for $\delta>0$ small enough, as $\lambda \rightarrow 0^{+}$

$$
\int_{B(\delta)} \chi(x) \cdot \exp (H(x) / \lambda) d x=\sum_{A_{\Delta}(\theta) \leq M} q_{\theta-1} \int_{B(\delta)} x^{\theta-1} \cdot \exp (H(x) / \lambda) d x+o\left(\lambda^{M}\right)
$$

where $A_{\Delta}(\theta)$ is the index of $\theta$ with respect to the Newton Diagram $\Delta$ of $H$.

2.3.3. Reduction of the phase. If $H \neq \hat{H}$, we will now prove in Proposition 3 , that the phase $H$ can be replaced by its principal part $\hat{H}$.

More precisely, in $\int_{B(\delta)} x^{\theta-1} \cdot \exp (H(x) / \lambda) d x=\int_{B(\delta)} x^{\theta-1} \cdot \exp \left(\frac{\hat{H}(x)+H(x)-\hat{H}(x)}{\lambda}\right) d x$, we must keep $\exp \left(\frac{\hat{H}(x)}{\lambda}\right)$ (because $\hat{H}$ includes the leading terms of the Taylor series of $H$ ), but we can expand $\exp \left(\frac{H(x)-\hat{H}(x)}{\lambda}\right)=\sum_{k=0}^{\infty} \frac{1}{k !}\left(\frac{H(x)-\hat{H}(x)}{\lambda}\right)^{k}$, and forget the terms corresponding to large $k$. 
Proposition 3. If $\theta \in \mathbb{N}^{* p}, M \in \mathbb{R}_{+}$, there exist polynomials $R_{k}$ and $M^{\prime} \in \mathbb{N}$, such that for $\delta>0$ small enough, as $\lambda \rightarrow 0^{+}$

$$
\int_{B(\delta)} x^{\theta-1} \cdot \exp (H(x) / \lambda) d x=\left[\sum_{k=0}^{M^{\prime}} \frac{1}{\lambda^{k}} \int_{B(\delta)} x^{\theta-1} R_{k}(x) \cdot \exp \left(\frac{\hat{H}(x)}{\lambda}\right) d x\right]+o\left(\lambda^{M}\right)
$$

$M^{\prime}$ is the integer part of $\frac{1}{\eta_{0}}\left(M-A_{\Delta}(\theta)\right)$.

Here $\quad \eta_{0}=\inf \left(A_{\Delta}(\mu)-1 ; \mu \in \Sigma(H) \backslash \Delta(H)\right)>0$

$R_{k}(x)=\frac{1}{k !}\left[\sum_{1<A_{\Delta}(\mu) \leq M+1} a_{\mu} x^{\mu}\right]^{k}$. In particular, $R_{0}=1$.

$\eta_{0}$ is the greatest number such that there is no point of the support of $H$ strictly between $\Delta$ and $\left(1+\eta_{0}\right) \Delta$.

Developing $R_{k}$ can lead to terms which are $o\left(\lambda^{M}\right)$. These terms can then be neglected. To find these terms, one must evaluate the indices of their corresponding integrals (see Lemma 4 (ii)).

Moreover, $k=0$ gives $\int_{B(\delta)} x^{\theta-1} \cdot \exp \left(\frac{\hat{H}(x)}{\lambda}\right) d x=o\left(\lambda^{A_{\Delta}(\theta)-\varepsilon}\right)$, for all $\varepsilon>0$.

If $k \geq 1$, for all $\varepsilon>0$

$$
\frac{1}{\lambda^{k}} \int_{B(\delta)} x^{\theta-1} R_{k}(x) \cdot \exp \left(\frac{\hat{H}(x)}{\lambda}\right) d x=o\left(\lambda^{A_{\Delta}(\theta)+k \eta_{0}-\varepsilon}\right)
$$

2.3.4. Extension of the domain. Proposition 4 allows to remove the bound $B(\delta)=\left\{x \in \mathbb{R}^{p} ;\|x\|<\delta\right\}$

Proposition 4. Let $\theta \in \mathbb{N}^{* p}, \hat{H}$ the non degenerate principal part of $H$. There exists $d^{\prime}>0$ such that as $\lambda \rightarrow 0^{+}$:

$$
\int_{B(\delta)} x^{\theta-1} \cdot \exp \left(\frac{\hat{H}(x)}{\lambda}\right) d x=\int_{\mathbb{R}^{p}} x^{\theta-1} \cdot \exp \left(\frac{\hat{H}(x)}{\lambda}\right) d x+O\left(e^{-d^{\prime} / \lambda}\right)
$$

It is clear now that if we apply successively Propositions 1 to 4, we obtain Theorem 1 (a).

2.3.5. Simplification of facets. We want now to transform $\int_{\mathbb{R}^{p}} x^{\theta-1} \cdot \exp \left(\frac{\hat{H}(x)}{\lambda}\right) d x$ to prove Theorem 1 (b). This transformation will be useful in particular to state Proposition 6.

If $\Delta$ has only one facet, $\int_{\mathbb{R}^{p}} x^{\theta-1} \cdot \exp \left(\frac{\hat{H}(x)}{\lambda}\right) d x$ is straightly computed with Proposition 7. If $\Delta$ has several facets, the support $\Sigma(\hat{H})$ of $\hat{H}$ includes the set $S=S_{\Delta}$ of vertices of $\Delta$, and possibly other points.

- If $\Sigma(\hat{H}) \backslash S$ is empty or included in one facet $F$, the equation (b) of Theorem 1 is obtained, since here $\hat{H}=H_{S \cup F}$.

- If not, $\Sigma(\hat{H}) \backslash S$ is included in $m$ different facets of $\Delta$ (and not less) with $m \geq 2$. To obtain Theorem 1 (b), we must diminish this number from $m$ to 1 . This is the use of Proposition 5, which enables us to decrease this number of facets from $m$ to $m-1$ (with $\Delta$ being not modified). It will allow to write our integrals as a linear combination of the same type of integrals $\int_{\mathbb{R}^{p}} x^{\theta^{\prime}-1} \cdot \exp \left(\frac{H_{B}(x)}{\lambda}\right) d x$ with $\Sigma\left(H_{B}\right) \backslash S$ included in $m-1$ facets. 
It means that Proposition 5 allows to "empty" one of the facet of the Newton diagram of $H$. Its repeated application will lead to integrals of the type $\int_{\mathbb{R}^{p}} x^{\theta^{\prime}-1} \cdot \exp \left(\frac{H_{S \cup F_{i}}(x)}{\lambda}\right) d x$ where $F_{i}$ is one facet of $\Delta, \theta^{\prime} \in \mathbb{N}^{* p}$.

The example studied in Section 6 can help to understand this proposition.

Proposition 5. Let $B$ be a subset of $\Sigma(\hat{H}) \backslash S_{\Delta}$ and $\theta \in \mathbb{N}^{* p}$.

We assume that $B$ is not included in one facet, i.e. $B$ is included in $m$ different facets $F_{1}, \ldots, F_{m}$ of $\Delta$ (and not less than $m$ ), with $m \geq 2$.

Then for any $M \in \mathbb{R}_{+}$, there exists $M " \in \mathbb{N}$ such that

$$
\begin{gathered}
\int_{\mathbb{R}^{p}} x^{\theta-1} \cdot \exp \left(\frac{H_{S \cup B}(x)}{\lambda}\right) d x \\
=o\left(\lambda^{M}\right)+\sum_{k_{1}=0}^{M "} \frac{1}{\lambda^{k_{1}}} \frac{1}{k_{1} !} \int_{\mathbb{R}^{p}} x^{\theta-1}\left(\sum_{\mu \in \Delta_{1}} a_{\mu} x^{\mu}\right)^{k_{1}} \exp \left(\frac{H_{S \cup B_{1}}(x)}{\lambda}\right) d x \\
+\sum_{k_{2}=0}^{M^{\prime \prime}} \frac{1}{\lambda^{k_{2}}} \frac{1}{k_{2} !} \int_{\mathbb{R}^{p}} x^{\theta-1}\left(\sum_{\mu \in \Delta_{2}} a_{\mu} x^{\mu}\right)^{k_{2}} \exp \left(\frac{H_{S \cup B_{2}}(x)}{\lambda}\right) d x \\
-\sum_{k_{1}=0}^{M^{\prime \prime}} \sum_{k_{2}=0}^{M^{\prime \prime}} \frac{1}{\lambda^{k_{1}+k_{2}}} \frac{1}{k_{1} ! k_{2} !} \int_{\mathbb{R}^{p}}^{k_{1}} x^{\theta-1}\left(\sum_{\mu \in \Delta_{1}} a_{\mu} x^{\mu}\right)^{k_{2}}\left(\sum_{\mu \in \Delta_{2}} a_{\mu} x^{\mu}\right)^{\exp \left(\frac{H_{S \cup B_{0}}(x)}{\lambda}\right) d x}
\end{gathered}
$$

where $\Delta_{i}=B \cap\left(F_{i} \backslash \cup_{j \neq i} F_{j}\right), i=1 ; 2$

and $B_{1}=B \backslash \Delta_{1}, B_{2}=B \backslash \Delta_{2}, B_{0}=B \backslash\left(\Delta_{1} \cup \Delta_{2}\right)$

$M$ " is the integer part of $\frac{1}{\eta}\left(M-A_{\Delta}(\theta)\right)$, where $\eta=\inf \left(\eta_{1}, \eta_{2}\right), \eta_{1}>0, \eta_{2}>0$ are defined for $i=1 ; 2$ as:

$$
\eta_{i}=\inf \left(A_{F_{j}}(\mu)-1 ; \mu \in \Delta_{i} \text { and } F_{j} \neq F_{i}\right) .
$$

Except a term $o\left(\lambda^{M}\right), \int_{\mathbb{R}^{p}} x^{\theta-1} \cdot \exp \left(\frac{\hat{H}(x)}{\lambda}\right) d x$ has been written as a linear combination of the same type of integrals, but with in exponent a polynomial $H_{S \cup B_{i}}$, where $B_{i}$ is included in at most $m-1$ facets. Reiterating this procedure leads to integrals $\int_{\mathbb{R}^{p}} x^{\theta-1} \cdot \exp \left(\frac{H_{S \cup F}(x)}{\lambda}\right) d x$ where $F$ is a facet of $\Delta$.

It means that the repeated application of Proposition 5 on $\int_{\mathbb{R}^{p}} x^{\theta-1} \cdot \exp \left(\frac{\hat{H}(x)}{\lambda}\right) d x$ gives Theorem $1(\mathrm{~b})$.

2.4. Removal of vertices $(p=2)$. According to Theorem 1 , we have just now to evaluate the expansion of $\int_{\mathbb{R}^{p}} x^{\theta-1} \cdot \exp \left(\frac{H_{S \cup F}(x)}{\lambda}\right) d x, F$ any facet of $\Delta, S$ the set of vertices of $\Delta$. If $S \subset F$, Proposition 7 leads to the calculation of the expansion.

For $p=2$, if some vertices don't belong to $F$ (i.e. $\Delta \neq F$ ) we show now that we can remove them. It is the matter of Proposition 6 , which allows to remove one of the bordering vertices of $\Delta$ (i.e. belonging to the boundary of $\mathcal{C}(\Delta)$ ). It is sufficient to study $\int_{\mathbb{R}_{+}^{2}} x^{\theta-1} \cdot \exp \left(\frac{H_{S \cup F}(x)}{\lambda}\right) d x$ because the behavior of the integrals on the other octans are of course similar. We will in fact study a more general integral, i.e.

$$
L(\theta, \gamma, P)=\int_{\mathbb{R}_{+}^{p}} x^{\theta-1}(\ln x)^{\gamma} \cdot \exp \left(\frac{P(x)}{\lambda}\right) d x
$$

where $(\theta, \gamma, P)$ satisfies the HP hypothesis, with $p=2$. 

it.

Section 6.6 gives an example of use of Proposition 6, which can help to understand

Proposition 6. Assume that $(\theta, \gamma, P)$ satisfies hypothesis $H P$, and that there exists a facet $F$ with $P(x)=\sum_{\mu \in S \cup F} a_{\mu} x^{\mu}$ i.e. the support of $P$ is included in $S \cup F$. Let $\nu$ be a bordering vertex of $\Delta$ (i.e. $\nu \notin \operatorname{int} \mathcal{C}(\Delta)), \nu \notin F$.

We set $P^{*}(x)=\sum_{\mu \in \Delta \backslash\{\nu\}} a_{\mu} x^{\mu}=\sum_{\mu \in \Delta^{*}} a_{\mu} x^{\mu}$ where $\Delta^{*}$ is the Newton diagram of $P^{*}$. Then for each $M \geq 0$, there exist constants $c_{i}^{\prime}$ and $c_{j, l}^{\prime}$ such that, as $\lambda \rightarrow 0^{+}$

$$
L(\theta, \gamma, P)=o\left(\lambda^{M}\right)+\sum_{i} c_{i}^{\prime} \lambda^{-i} L\left(\theta_{i} ; \gamma_{i} ; P^{*}\right)+\sum_{j, l} c_{j, l}^{\prime} \lambda^{j}(\ln \lambda)^{l}
$$

where the sums have only a finite number of terms, $i \in \mathbb{N}, l \in \mathbb{N} . \Delta^{*}$ contains one vertex (and one facet) less than $\Delta .\left(\theta_{i} ; \gamma_{i} ; P^{*}\right)$ satisfy the HP hypothesis.

Here are the main steps of the calculus:

1) Write $L(\theta, \gamma, P)$ as a linear combination of $M_{B}$, where $M_{B}$ is given in Equation (12).

2) Put

$$
M_{B}=\int_{\mathbb{R}_{+}}\left[\int_{\mathbb{R}_{+}} \phi_{\lambda}(w ; t) f_{\lambda}(w ; t) d t\right] d w
$$

with $\phi_{\lambda}$ and $f_{\lambda}$ defined in (19).

3) Use the asymptotic expansion of $f_{\lambda}$ as $t \rightarrow+\infty$ in (20) to obtain the decomposition $M_{B}=I_{1}+I_{2}+I_{3}$ given in $(22)$, and the value of $a_{s}(\lambda ; w)$.

4) Set $n=c^{-}+\left(-\beta_{2}(\nu)\right) \bar{R}$, where $\bar{R}$ is the integer part of $1+\frac{M-A_{\Delta^{*}}\left(b^{\prime}\right)}{A_{\Delta^{*}}(\nu)-1}$ to have $I_{3}=o\left(\lambda^{M}\right)$.

5) Compute $I_{1}=\int_{\mathbb{R}_{+}}\left[\sum_{s=0}^{n-1} a_{s}(\lambda, w)<(\ln t)_{+}^{k} t_{+}^{-s-\alpha} ; \phi_{\lambda}>\right] d w$, then come back, if necessary, to the variable $x$.

6) Compute the $c_{s}(\lambda, w)$ and then $I_{2}=-\int_{\mathbb{R}_{+}}\left[\sum_{s=1}^{n} c_{s}(\lambda, w)<\delta^{(s-1)} ; \phi_{\lambda}>\right] d w$

7) Write the final expansion of $L(\theta, \gamma, P)$.

2.5. Newton Diagram with a unique facet $(p \geq 2)$. If $p=2$, Proposition 6 shows how to remove one vertex $\nu$, with $\nu / \in F$. Its repeated application will lead to integrals $L\left(\theta, \gamma, P_{F}\right)$, i.e. where the Newton Diagram has only one facet $F$. Proposition 7 deals with this case for any $p$.

Proposition 7. Assume that $(\theta, \gamma, P)$ satisfies Hypothesis HP, and that the Newton Diagram $\Delta$ of $P$ contains only one facet $F$ (i.e. $\Delta=F$ ). Then there exist constants $c_{l}$ such that :

$$
L(\theta, \gamma, P)=\lambda^{A_{F}(\theta)} \sum_{l=0}^{\gamma_{1}+. .+\gamma_{p}} c_{l}(\ln \lambda)^{l}
$$

$c_{l}$ is given by formula (40).

\section{Proofs of Propositions 1 to 5.}

\subsection{Proofs of Lemmas 1 to 3.}


Proof of Lemma 1. Let $l(\mu)=\sum d_{i} \mu_{i}=1$ be the equation of a supporting hyperplane of $F$. For any $\mu \in \Pi, l(\mu) \geq 1$ and $l(\mu)=1 \Longleftrightarrow \mu \in F$.

For $M_{0}$ large enough, since $H$ is convenient, as $x \rightarrow 0$

$$
H(x)=\sum_{l(\mu) \leq M_{0}} a_{\mu} x^{\mu}+o\left(\sum_{\mu \in S_{\Delta}} x^{\mu}\right)
$$

Now fix $x \in \mathbb{R}^{p}$, and let $\varepsilon>0$, with $\varepsilon \rightarrow 0$

$$
\begin{aligned}
H\left(\epsilon^{d_{1}} x_{1}, . ., \epsilon^{d_{p}} x_{p}\right) & =\sum_{l(\mu) \leq M_{0}} a_{\mu} \prod_{j=1}^{p}\left(\varepsilon^{d_{j}} x_{j}\right)^{\mu_{j}}+o\left(\sum_{\mu \in S_{\Delta}} \prod_{j=1}^{p}\left(\varepsilon^{d_{j}} x_{j}\right)^{\mu_{j}}\right) \\
& =\sum_{l(\mu) \leq M_{0}} a_{\mu} \varepsilon^{l(\mu)} x^{\mu}+o\left(\sum_{\mu \in S_{\Delta}} \varepsilon^{l(\mu)} x^{\mu}\right)
\end{aligned}
$$

with $l(\mu) \geq 1$ if $\mu \in S_{\Delta}$, so as $\varepsilon \rightarrow 0$

$$
\begin{aligned}
H\left(\epsilon^{d_{1}} x_{1}, . ., \epsilon^{d_{p}} x_{p}\right) & =\sum_{l(\mu) \leq M_{0}} a_{\mu} \varepsilon^{l(\mu)} x^{\mu}+o(\varepsilon) \\
& =\sum_{l(\mu)=1} a_{\mu} \varepsilon^{l(\mu)} x^{\mu}+\sum_{1<l(\mu) \leq M_{0}} a_{\mu} \varepsilon^{l(\mu)} x^{\mu}+o(\varepsilon) \\
& =\varepsilon H_{F}(x)+o(\varepsilon)
\end{aligned}
$$

From $H \leq 0$ we conclude that $H_{F}(x) \leq 0$.

Proof of Lemma 2. For $\mu^{\prime}$ a vertex of $\Pi, F=\left\{\mu^{\prime}\right\}$ is a face of $\Delta$, then it satisfies Lemma 1 :

$H_{F}(x) \leq 0, \forall x \in \mathbb{R}^{p}$, i.e. $a_{\mu^{\prime}} x^{\mu^{\prime}} \leq 0, \forall x \in \mathbb{R}^{p}$.

Thus $a_{\mu^{\prime}} \leq 0$, and we even have $a_{\mu^{\prime}}<0$ since $\mu^{\prime} \in \Pi$.

Let us show that $\mu_{i}^{\prime}$ is even.

$a_{\mu^{\prime}} \cdot(1, . .1,-1,1, . ., 1)^{\mu^{\prime}} \leq 0$ (here -1 is at the $i^{t h}$ place),

i.e. $a_{\mu^{\prime}}(-1)^{\mu_{i}^{\prime}} \leq 0$, which becomes $(-1)^{\mu_{i}^{\prime}} \geq 0$, thus $\mu_{i}^{\prime}$ is even.

Proof of Lemma 3.

- $(i) \Rightarrow($ iii $)$ ?

We know that $H_{F} \leq 0$. If $H_{F}$ vanishes at $x \in \mathbb{R}^{* p}$, then $\nabla H_{F}$ vanishes at $x$. Then $\nabla H_{F} \neq 0$ on $\mathbb{R}^{* p} \Rightarrow H_{F}$ never vanishes on $\mathbb{R}^{* p}$.

- $($ iii $) \Rightarrow(i)$ ?

$F$ is in the hyperplane $\sum_{i=1}^{p} d_{i} \mu_{i}=1$.

For $\epsilon>0, H_{F}\left(\epsilon^{d_{1}} x_{1}, . ., \epsilon^{d_{p}} x_{p}\right)=\sum_{\mu \in F} a_{\mu}\left(\epsilon^{d_{1}} x_{1}\right)^{\mu_{1}} \ldots\left(\epsilon^{d_{p}} x_{p}\right)^{\mu_{p}}=\epsilon \cdot H_{F}(x)$

$\nabla H_{F}\left(\epsilon^{d_{1}} x_{1}, . ., \epsilon^{d_{p}} x_{p}\right)=\epsilon \nabla H_{F}(x)$ then $\nabla H_{F}(x)=0 \Rightarrow$ $\nabla H_{F}\left(\epsilon^{d_{1}} x_{1}, . ., \epsilon^{d_{p}} x_{p}\right)=0$

Thus if $\nabla H_{F}$ vanishes on a point of $\mathbb{R}^{* p}$, it vanishes on a curve of limit 0 in $\mathbb{R}^{* p}$ (with $H_{F}(0)=0$ ). Then $H_{F}(x)=0$ on this curve.

- $($ ii $) \Rightarrow($ iii $)$ ? Setting $g(x)=\hat{H}(x)+c \cdot \sum_{\mu \in S} x^{\mu}$ and $g_{F}(x)=H_{F}(x)+$ c. $\sum_{S \cap F} x^{\mu}$, we see that $g$ satisfies the same hypothesis than $H$, so $g_{F} \leq 0$, where $H_{F}<g_{F} \leq 0$ in a neighborhood of 0 on $\mathbb{R}^{* p}$ hence (iii) is true.

- $($ iii $) \Rightarrow($ ii $)$ : just apply in Vasil'ev [13], the Gindikin's remark in the proof of Theorem $1.5(2)$ with $f=-\hat{H}$.

- $(i v) \Rightarrow(i i i)$ is obvious.

- $(i i i) \Rightarrow(i v)$ ? $(i i)$ and $(i i i)$ together imply $(i v)$. 
3.2. Proof of Lemma 4. Before proving Lemma 4, we give a change of variables that will be useful afterwards.

3.2.1. Facet-system change of variables. A facet-system $\sigma=(\mu(1), . ., \mu(p))$ is a system of $p$ independent vertices of a same facet $F$ of $\Delta$, the $\mu(i) \in \mathbb{N}^{p}$. Let us define the following change of variables, associated to the facet-system $\sigma=(\mu(1), . ., \mu(p))$ :

$x=\left(x_{1}, . ., x_{p}\right) \mapsto v^{(\sigma)}=\left(v_{1}^{(\sigma)}, . ., v_{p}^{(\sigma)}\right)$ or simply denoted by $v$, where $v_{i}=x^{\frac{1}{D} \mu(i)}$ for $x \in \mathbb{R}_{+}^{* p}$ with $D=\operatorname{det}(\mu(1), . . \mu(p))>0$ (otherwise the order of these points is modified).

An elementary calculation gives the Jacobian $J$ of this change of variables :

$$
J=D^{p-1} \cdot \frac{\prod x_{i}}{\prod v_{i}} \quad \text { i.e. } \quad \frac{d x}{\prod x_{i}}=D^{p-1} \cdot \frac{d v}{\prod v_{i}}
$$

writing $d x=d x_{1} . . d x_{p}, d v=d v_{1} . . d v_{p}$

Lemma 5 gives supplementary information on this change of variables, and links it to the index $A_{F}(\mu)$.

Lemma 5. Let $\sigma=(\mu(1), . ., \mu(p))$ be a facet-system, the $\mu(i)$ in $F$ a given facet of $\Delta$.

(i) Then for each $\mu \in \mathbb{R}^{p}$, there exists $\beta(\mu) \in \mathbb{R}^{p}$ such that, for any $x \in \mathbb{R}_{+}^{* p}$ :

$$
\begin{gathered}
x^{\mu}=v^{\beta(\mu)}=v_{1}^{\beta_{1}(\mu)} . . v_{p}^{\beta_{p}(\mu)} \text { with } \quad \beta(\mu)=\left(\beta_{1}, . ., \beta_{p}(\mu)\right) \\
\text { and } \beta_{i}(\mu)=\operatorname{det}(\mu(1), . ., \mu(i-1), \mu, \mu(i+1), . ., \mu(p)), \quad \mu=\frac{1}{D} \cdot \sum_{i=1}^{p} \beta_{i}(\mu) . \mu(i)
\end{gathered}
$$

(ii) Moreover, $A_{F}(\mu)=\frac{1}{D} \cdot \sum_{i=1}^{p} \beta_{i}(\mu),\left(A_{F}(\mu)\right.$ is the index of $\mu$ with respect to $\left.F\right)$. $F$ is a part of the hyperplane of equation $\beta_{1}(\mu)+. .+\beta_{p}(\mu)=D$ so : for $\mu \in \Pi,\left(\mu \in F \Longleftrightarrow A_{F}(\mu)=1\right)$ and $\left(\mu \in \Pi \backslash F \Longleftrightarrow A_{F}(\mu)>1\right)$. $\mu \in \operatorname{Conv}(\mu(1), . ., \mu(p)) \Longleftrightarrow\left(A_{F}(\mu)=1\right.$ and $\left.\beta_{i}(\mu) \geq 0, \forall i\right)$

3.2.2. Proof of Lemma 5. - First we prove $(i)$. We want $x^{\mu}=v^{\beta(\mu)}$, where

$$
v^{\beta(\mu)}=\prod_{j=1}^{p}\left(v_{j}\right)^{\beta_{j}(\mu)}=\prod_{j=1}^{p}\left(x^{\frac{1}{D} \mu(j)}\right)^{\beta_{j}(\mu)}
$$

then we must have

$$
\mu=\frac{1}{D} \sum_{j=1}^{p} \beta_{j}(\mu) \cdot \mu(j)
$$

i.e. we want that

$$
D \cdot \mu_{i}=\beta_{1}(\mu) \cdot \mu_{i}(1)+. .+\beta_{p}(\mu) \cdot \mu_{i}(p), \forall i \in[1, p],
$$

which is a linear system of $p$ equations with $p$ variables $\beta_{1}(\mu), \ldots, \beta_{p}(\mu)$, with solutions

$$
\beta_{j}(\mu)=\frac{\operatorname{det}(\mu(1), . ., \mu(j-1), D \mu, \mu(j+1), . ., \mu(p))}{\operatorname{det}(\mu(1), . ., \mu(p))}
$$


with

$D=\operatorname{det}(\mu(1), . ., \mu(p))$, i.e. :

$$
\beta_{j}(\mu)=\operatorname{det}(\mu(1), . ., \mu(j-1), \mu, \mu(j+1), . ., \mu(p))
$$

- It remains to prove $(i i)$.

$\mu=\frac{1}{D} \sum \beta_{j}(\mu) \cdot \mu(j)$ applied to the $\mu=\mu(i)$ gives that the hyperplane including the $\mu(i)$ has the equation $\frac{1}{D} \sum \beta_{j}(\mu)=1$. We have defined $A_{F}(\mu)$ as the linear form such that $A_{F}(\mu)=1$ is the equation of the hyperplane including $F$.

Thus $A_{F}(\mu)=\frac{1}{D} \sum \beta_{j}(\mu)$.

3.2.3. Proof of Lemma 4. 1) Let us first prove this lemma for $\theta \in \operatorname{int}(\mathcal{C}(\sigma))$, $\sigma$ a facet-system of a facet $F$ of $\Delta$. We apply the transformation $x \mapsto v^{(\sigma)}$ for the facet-system $\sigma=(\mu(1), . ., \mu(p))$ of $F$.

Putting $D=\operatorname{det}(\mu(1), . ., \mu(p))$ and applying Lemma 5, we obtain :

$$
L_{B}(\theta, \gamma, P)=\int_{B} \exp \left(\frac{1}{\lambda} \sum_{\mu \in \Delta} a_{\mu} v^{\beta(\mu)}\right) \cdot v^{\beta(\theta)-1} \prod_{j=1}^{p}\left(\ln \left(v^{\beta\left(e_{j}\right)}\right)\right)^{\gamma_{j}} D^{p-1} d v
$$

We set now $u_{i}=\lambda^{-\frac{1}{D}} v_{i}$. We obtain

$$
v^{\beta(\mu)}=\prod_{i=1}^{p} v_{i}^{\beta_{i}(\mu)}=\prod_{i=1}^{p}\left(\lambda^{\frac{1}{D}} u_{i}\right)^{\beta_{i}(\mu)}=\lambda^{A_{F}(\mu)} u^{\beta(\mu)}
$$

thus

$$
\begin{gathered}
L_{B}(\theta, \gamma, P)=\lambda^{A_{F}(\theta)} \int_{B} \exp \left(\sum_{\mu \in \Delta} a_{\mu} \lambda^{A_{F}(\mu)-1} u^{\beta(\mu)}\right) \cdot u^{\beta(\theta)-1}\left(\ln \left(\lambda^{A_{F}\left(e_{1}\right)} u^{\beta\left(e_{1}\right)}\right)\right)^{\gamma_{1}} \\
\ldots\left(\ln \left(\lambda^{A_{F}\left(e_{p}\right)} u^{\beta\left(e_{p}\right)}\right)\right)^{\gamma_{p}} D^{p-1} d u \\
=\sum_{k_{1}=0}^{\gamma_{1}} \ldots \sum_{k_{p}=0}^{\gamma_{p}}\left(\begin{array}{c}
\gamma_{1} \\
k_{1}
\end{array}\right) \ldots\left(\begin{array}{c}
\gamma_{p} \\
k_{p}
\end{array}\right)\left(\ln \lambda^{A_{F}\left(e_{1}\right)}\right)^{k_{1}} \ldots\left(\ln \lambda^{A_{F}\left(e_{p}\right)}\right)^{k_{p}} \cdot \lambda^{A_{F}(\theta)} \\
\int_{B} \exp \left(\sum_{\mu \in \Delta} a_{\mu} \lambda^{A_{F}(\mu)-1} u^{\beta(\mu)}\right) \cdot u^{\beta(\theta)-1}\left(\ln u^{\beta\left(e_{1}\right)}\right)^{\gamma_{1}-k_{1}} \ldots\left(\ln u^{\beta\left(e_{p}\right)}\right)^{\gamma_{p}-k_{p}} D^{p-1} d u
\end{gathered}
$$

where in this last integral, $A_{F}(\mu)-1=0$ for $\mu \in F$, and $A_{F}(\mu)-1>0$ for $\mu \in \Delta \backslash F$. $\beta_{i}(\theta)>0$ for every $i$ since $\theta \in \operatorname{int}(\mathcal{C}(\sigma))$.

Thus, by dominated convergence, each integral in the right hand side of (2) tends to:

$$
\int_{B} \exp \left(\sum_{\mu \in F} a_{\mu} u^{\beta(\mu)}\right) \cdot u^{\beta(\theta)-1}\left(\ln u^{\beta\left(e_{1}\right)}\right)^{\gamma_{1}-k_{1}} \ldots\left(\ln u^{\beta\left(e_{p}\right)}\right)^{\gamma_{p}-k_{p}} D^{p-1} d u
$$

Let us briefly explain why there is dominated convergence here.

Since $P$ is nondegenerate, there is $c>0$ such that

$$
P(x) \leq-c \sum_{\mu \in S} x^{\mu} \leq-c \sum_{\mu \in S \cap F} x^{\mu}
$$


Then with our change of variables

$$
\begin{gathered}
\sum_{\mu \in \Delta} a_{\mu} v^{\beta(\mu)} \leq-c \sum_{\mu \in S \cap F} v^{\beta(\mu)} \leq-c \sum_{i=1}^{p} v^{\beta(\mu(i))}=-c \sum_{i=1}^{p} v_{i}^{D} \\
\sum_{\mu \in \Delta} a_{\mu} \lambda^{A_{F}(\mu)-1} u^{\beta(\mu)} \leq-\frac{c}{\lambda} \sum_{i=1}^{p}\left(\lambda^{\frac{1}{D}} u_{i}\right)^{D}=-c \sum_{i=1}^{p} u_{i}^{D}
\end{gathered}
$$

and

$$
\begin{aligned}
& u^{\beta(\theta)-1} \exp \left(\sum_{\mu \in \Delta} a_{\mu} \lambda^{A_{F}(\mu)-1} u^{\beta(\mu)}\right)\left|\left(\ln u^{\beta\left(e_{1}\right)}\right)^{\gamma_{1}-k_{1}} \ldots\left(\ln u^{\beta\left(e_{p}\right)}\right)^{\gamma_{p}-k_{p}}\right| \\
& \leq u^{\beta(\theta)-1} \exp \left(-c \sum_{i=1}^{p} u_{i}^{D}\right)\left|\left(\ln u^{\beta\left(e_{1}\right)}\right)^{\gamma_{1}-k_{1}} \ldots\left(\ln u^{\beta\left(e_{p}\right)}\right)^{\gamma_{p}-k_{p}}\right|
\end{aligned}
$$

which is clearly integrable on $\mathbb{R}_{+}^{p}$ since $u^{\beta(\theta)-1}=\prod_{i=1}^{p} u_{i}^{\beta_{i}(\theta)}$, where $\beta_{i}(\theta)>0$. Hence the dominated convergence. An equivalent of $L$ is then obtained by taking in (2) the greatest possible values for the exponents of $\ln \lambda$, i.e. :

$$
\begin{aligned}
L_{B} & \sim \lambda^{A_{F}(\theta)}(\ln \lambda)^{\gamma_{1}+. .+\gamma_{p}} \cdot \prod_{i=1}^{p}\left(A_{F}\left(e_{i}\right)\right)^{\gamma_{i}} \int_{B} \exp \left(\sum_{\mu \in F} a_{\mu} u^{\beta(\mu)}\right) \cdot u^{\beta(\theta)-1} D^{p-1} d u \\
& =\lambda^{A_{\Delta}(\theta)}(\ln \lambda)^{\gamma_{1}+. .+\gamma_{p}} \cdot \prod_{i=1}^{p}\left(A_{F}\left(e_{i}\right)\right)^{\gamma_{i}} \int_{B} \exp \left(P_{F}(x)\right) \cdot x^{\theta-1} d x
\end{aligned}
$$

2) We now prove (i) and (ii) for any $\theta$ such that $(\theta, \gamma, P)$ satisfy HP.

If $\theta$ is not in the interior of the cone generated by $p$ vertices of a same facet $F$ of $\Delta$, then $\theta$ is in the intersection of several such cones. We can find $\theta^{\prime}$ and $\theta^{\prime \prime}$ satisfying this property, with $\theta \in] \theta^{\prime}, \theta^{\prime \prime}[$, arbitrarily close to $\theta$. Thus

$$
\begin{aligned}
\left|L_{B}(\theta, \gamma, P)\right| & \leq \int_{B} x^{\theta-1}|\ln x|^{\gamma} \exp \left(\frac{P(x)}{\lambda}\right) d x \\
& \leq \int_{B} x^{\theta^{\prime}-1}|\ln x|^{\gamma} \exp \left(\frac{P(x)}{\lambda}\right) d x+\int_{B} x^{\theta^{\prime \prime}-1}|\ln x|^{\gamma} \exp \left(\frac{P(x)}{\lambda}\right) d x
\end{aligned}
$$

where these 2 integrals are convergent according to Lemma 4 (i) applied on $\theta$ and $\theta^{\prime}$. Hence $L_{B}$ is convergent.

Moreover, for any $\varepsilon>0$,

$$
\begin{aligned}
\left|L_{B}(\theta, \gamma, P)\right| & \leq \int_{B} x^{\theta^{\prime}-1}|\ln x|^{\gamma} \exp \left(\frac{P(x)}{\lambda}\right) d x+\int_{B} x^{\theta^{\prime \prime}-1}|\ln x|^{\gamma} \exp \left(\frac{P(x)}{\lambda}\right) d x \\
& \leq \text { const. }\left(\lambda^{A_{\Delta}\left(\theta^{\prime}\right)-\frac{\varepsilon}{2}}+\lambda^{A_{\Delta}\left(\theta^{\prime \prime}\right)-\frac{\varepsilon}{2}}\right)
\end{aligned}
$$

according to Lemma 4 (ii) applied on $\theta$ and $\theta^{\prime}$.

As $\theta^{\prime}$ and $\theta "$ can be chosen arbitrarily close to $\theta$ (but not equal to $\theta$ ), then we can chose them such that $A_{\Delta}\left(\theta^{\prime}\right)>A_{\Delta}(\theta)-\frac{\varepsilon}{2}$ and $A_{\Delta}\left(\theta^{\prime \prime}\right)>A_{\Delta}(\theta)-\frac{\varepsilon}{2}$. Hence $L_{B}(\theta, \gamma, P)=o\left(\lambda^{A_{\Delta}(\theta)-\varepsilon}\right)$ 
3) Let us finally prove (iii) for $\theta \in \operatorname{int} \mathcal{C}(F)$, but such that there exists no facetsystem $\sigma$ with $\theta \in \operatorname{int} \mathcal{C}(\sigma)$. In that case, there exist $\theta^{\prime}, \theta^{\prime \prime}$ such that $\theta^{\prime} \in \operatorname{int} \mathcal{C}\left(\sigma^{\prime}\right)$ and $\left.\theta^{\prime \prime} \in \operatorname{int} \mathcal{C}\left(\sigma^{\prime \prime}\right), \theta \in\right] \theta^{\prime}, \theta^{\prime \prime}\left[, \sigma^{\prime}\right.$ and $\sigma^{\prime \prime}$ are 2 different facet-systems of $F$ with $A_{\Delta}\left(\theta^{\prime}\right)=A_{\Delta}\left(\theta^{\prime \prime}\right)=A_{\Delta}(\theta)$.

Let $\theta_{n}^{\prime}=\theta+\frac{\left(\theta^{\prime}-\theta\right)}{n}$ and $\theta_{n}^{\prime \prime}=\theta+\frac{\left(\theta^{\prime \prime}-\theta\right)}{n}$.

We see easily that for any $n \in \mathbb{N}^{*}, \theta_{n}^{\prime} \in \operatorname{int} \mathcal{C}\left(\sigma^{\prime}\right)$ and $\left.\theta_{n}^{\prime \prime} \in \operatorname{int} \mathcal{C}\left(\sigma^{\prime \prime}\right), \theta \in\right] \theta_{n}^{\prime}, \theta_{n}^{\prime \prime}[$, $A_{\Delta}\left(\theta_{n}^{\prime}\right)=A_{\Delta}\left(\theta_{n}^{\prime \prime}\right)=A_{\Delta}(\theta)$ and $\lim _{n} \theta_{n}^{\prime}=\lim _{n} \theta_{n}^{\prime \prime}=\theta$

Let $B_{1}=\left\{x \in B ; x^{\theta^{\prime}}(\ln x)^{\gamma}<x^{\theta}(\ln x)^{\gamma}<x^{\theta^{\prime \prime}}(\ln x)^{\gamma}\right\}$ and $B_{2}=\{x \in$ $\left.B ; x^{\theta^{\prime \prime}}(\ln x)^{\gamma}<x^{\theta}(\ln x)^{\gamma}<x^{\theta^{\prime}}(\ln x)^{\gamma}\right\}$.

As $\theta \in] \theta^{\prime}, \theta^{\prime \prime}\left[, \theta \neq \theta^{\prime}\right.$ and $\theta \neq \theta^{\prime \prime}$.

We have $L_{B}=L_{B_{1}}+L_{B_{2}}$ (i.e. we can't have for instance $x^{\theta^{\prime}}(\ln x)^{\gamma}<x^{\theta^{\prime \prime}}(\ln x)^{\gamma}<$ $\left.x^{\theta}(\ln x)^{\gamma}\right)$

$$
x^{\theta_{n}^{\prime}}<x^{\theta} \Leftrightarrow x^{\theta+\frac{1}{n}\left(\theta^{\prime}-\theta\right)}<x^{\theta}
$$

i.e. $x^{\theta_{n}^{\prime}}<x^{\theta} \Leftrightarrow x^{\theta^{\prime}}<x^{\theta}$

thus for any $n$

$$
\begin{aligned}
& B_{1}=\left\{x \in B ; x^{\theta_{n}^{\prime}}(\ln x)^{\gamma}<x^{\theta}(\ln x)^{\gamma}<x^{\theta_{n}^{\prime \prime}}(\ln x)^{\gamma}\right\} \\
& B_{2}=\left\{x \in B ; x^{\theta_{n} \prime}(\ln x)^{\gamma}<x^{\theta}(\ln x)^{\gamma}<x^{\theta_{n}^{\prime}}(\ln x)^{\gamma}\right\}
\end{aligned}
$$

which implies that

$$
L_{B_{1}}\left(\theta_{n}^{\prime}, \gamma, P\right) \leq L_{B_{1}}(\theta, \gamma, P) \leq L_{B_{1}}\left(\theta_{n}^{\prime \prime}, \gamma, P\right)
$$

and $L_{B_{2}}\left(\theta_{n}^{\prime \prime}, \gamma, P\right) \leq L_{B_{2}}(\theta, \gamma, P) \leq L_{B_{2}}\left(\theta_{n}^{\prime}, \gamma, P\right)$

For any $n \in \mathbb{N}^{*}$ stated, $\lambda>0$,

$$
\frac{L_{B_{1}}\left(\theta_{n}^{\prime}, \gamma, P\right)}{C_{\gamma}(F) \cdot \lambda^{A_{\Delta}(\theta)}(\ln \lambda)^{\sum \gamma_{i}}} \leq \frac{L_{B_{1}}(\theta, \gamma, P)}{C_{\gamma}(F) \cdot \lambda^{A_{\Delta}(\theta)}(\ln \lambda)^{\sum \gamma_{i}}} \leq \frac{L_{B_{1}}\left(\theta_{n}^{\prime \prime}, \gamma, P\right)}{C_{\gamma}(F) \cdot \lambda^{A_{\Delta}(\theta)}(\ln \lambda)^{\sum \gamma_{i}}}
$$

where the left hand side tends to $\int_{B_{1}}|x|^{\theta_{n}^{\prime}-1} e^{P_{F}(x)} d x$ as $\lambda \rightarrow 0^{+}$ and the right hand side tends to $\int_{B_{1}}|x|^{\theta_{n}^{\prime \prime}-1} e^{P_{F}(x)} d x$ as $\lambda \rightarrow 0^{+}$ according to Lemma 4 (iii) applied to $\theta_{n}^{\prime}$ and $\theta_{n}^{\prime \prime}$.

So, for any $n \in \mathbb{N}^{*}$,

$$
\begin{aligned}
\int_{B_{1}}|x|^{\theta_{n}^{\prime}-1} e^{P_{F}(x)} d x & \leq \lim _{\lambda \rightarrow 0^{+}} \inf \frac{L_{B_{1}}(\theta, \gamma, P)}{C_{\gamma}(F) \cdot \lambda^{A_{\Delta}(\theta)}(\ln \lambda)^{\sum \gamma_{i}}} \\
\lim _{\lambda \rightarrow 0^{+}} \sup \frac{L_{B_{1}}(\theta, \gamma, P)}{C_{\gamma}(F) \cdot \lambda^{A_{\Delta}(\theta)}(\ln \lambda)^{\sum \gamma_{i}}} & \leq \int_{B_{1}}|x|^{\theta_{n}^{\prime \prime}-1} e^{P_{F}(x)} d x
\end{aligned}
$$

This is true for any $n \in \mathbb{N}^{*}$, thus since $\theta_{n}^{\prime} \rightarrow \theta$ and $\theta_{n}^{\prime \prime} \rightarrow \theta($ as $n \rightarrow+\infty)$ :

$$
\begin{aligned}
\int_{B_{1}}|x|^{\theta-1} e^{P_{F}(x)} d x & \leq \lim _{\lambda \rightarrow 0^{+}} \inf \frac{L_{B_{1}}(\theta, \gamma, P)}{C_{\gamma}(F) \cdot \lambda^{A_{\Delta}(\theta)}(\ln \lambda)^{\sum \gamma_{i}}} \\
\lim _{\lambda \rightarrow 0^{+}} \sup \frac{L_{B_{1}}(\theta, \gamma, P)}{C_{\gamma}(F) \cdot \lambda^{A_{\Delta}(\theta)}(\ln \lambda)^{\sum \gamma_{i}}} & \leq \int_{B_{1}}|x|^{\theta-1} e^{P_{F}(x)} d x
\end{aligned}
$$

This implies that

$$
\lim _{\lambda \rightarrow 0^{+}} \frac{L_{B_{1}}(\theta, \gamma, P)}{C_{\gamma}(F) \cdot \lambda^{A_{\Delta}(\theta)}(\ln \lambda)^{\sum \gamma_{i}}}=\int_{B_{1}}|x|^{\theta-1} e^{P_{F}(x)} d x
$$

The same result holds on $B_{2}$ (interverting $\theta_{n}^{\prime}$ and $\theta_{n}^{\prime \prime}$ ) so

$$
\lim _{\lambda \rightarrow 0^{+}} \frac{L_{B}(\theta, \gamma, P)}{C_{\gamma}(F) \cdot \lambda^{A_{\Delta}(\theta)}(\ln \lambda)^{\sum \gamma_{i}}}=\int_{B}|x|^{\theta-1} e^{P_{F}(x)} d x
$$


This ends the proof of Lemma 4.

3.3. Proof of Proposition 1. $K \backslash B(\delta)$ is a compact set not including 0 , thus $H$ reaches there its maximum and this one is strictly lower than $H(0)=\max H=0$. In other words, there exists $d>0$ such that, for $x \in K \backslash B(\delta), H(x)<-d<0$. $\chi$ is continuous on the compact set $K \backslash B(\delta)$ hence $|\chi(x)| \leq M_{0}$, for a $M_{0} \in \mathbb{R}$.

$$
\left|\int_{K \backslash B(\delta)} \chi(x) \cdot \exp (H(x) / \lambda) d x\right| \leq \exp \left(\frac{-d}{\lambda}\right) \int_{K \backslash B(\delta)} M_{0} d x
$$

3.4. Proof of Proposition 2. $S$ is the set of vertices of $\Delta(H), A(\theta)=A_{\Delta}(\theta)$ the index of $\theta$ with respect to $\Delta(H)$. We want to show that, as $\lambda \rightarrow 0^{+}$

$$
\int_{B(\delta)}\left[\chi(x)-\sum_{A(\theta) \leq M} x^{\theta-1} q_{\theta-1}\right] \cdot \exp \left(\frac{H(x)}{\lambda}\right) d x=o\left(\lambda^{M}\right)
$$

According to the Taylor series of $\chi$ at 0

$$
\chi(x)-\sum_{A(\theta-1) \leq M} q_{\theta-1} x^{\theta-1}=\chi(x)-\sum_{A(\theta) \leq M} q_{\theta} x^{\theta}=o\left(\sum_{\mu \in S} x^{\mu . M}\right)
$$

thus it is sufficient to prove that, for $\delta$ small enough

$$
\int_{B(\delta)} x^{\mu \cdot M} \cdot \exp \left(\frac{H(x)}{\lambda}\right) d x=o\left(\lambda^{M}\right) \quad \text { for } \quad \mu \in S
$$

and that

$$
\int_{B(\delta)} x^{\theta-1} \cdot \exp \left(\frac{H(x)}{\lambda}\right) d x=o\left(\lambda^{M}\right) \quad \text { for } \quad A(\theta-1) \leq M<A(\theta)
$$

Let us apply Proposition 3.

The first term (i.e. the one of least degree in $\lambda$ ) of $\int_{B(\delta)} x^{\mu \cdot M} \cdot \exp \left(\frac{H(x)}{\lambda}\right) d x$ (respectively of $\left.\int_{B(\delta)} x^{\theta-1} \cdot \exp \left(\frac{H(x)}{\lambda}\right) d x\right)$ in the formulation provided by Proposition 3 is $\int_{B(\delta)} x^{\mu \cdot M} \cdot \exp \left(\frac{\hat{H}(x)}{\lambda}\right) d x$ (respectively $\left.\int_{B(\delta)} x^{\theta-1} \cdot \exp \left(\frac{\hat{H}(x)}{\lambda}\right) d x\right)$

Now, by Lemma 4 , it is known that this last integral is $o\left(\lambda^{M}\right)$ because for $\mu \in S$, $A(\mu M+1)>A(\mu M)=M \cdot A(\mu)=M$ (respectively $A(\theta)>M)$.

3.5. Proof of Proposition 3. Let $M^{\prime} \in \mathbb{N}$. For all $y \in \mathbb{R}$, let $S_{M^{\prime}}(y)=$ $e^{y}-\sum_{k=0}^{M^{\prime}} \frac{y^{k}}{k !}$.

We have $\left|S_{M^{\prime}}(y)\right| \leq|y|^{M^{\prime}+1} e^{|y|}$

$$
\begin{aligned}
\int_{B(\delta)} x^{\theta-1} \cdot \exp \left(\frac{H(x)}{\lambda}\right) d x=\int_{B(\delta)} x^{\theta-1} \cdot \exp \left(\frac{\hat{H}(x)}{\lambda}\right) \cdot \exp \left(\frac{H(x)-\hat{H}(x)}{\lambda}\right) d x \\
=\sum_{k=0}^{M^{\prime}} \frac{1}{k !} \int_{B(\delta)} x^{\theta-1} \cdot \exp \left(\frac{\hat{H}(x)}{\lambda}\right) \cdot\left(\frac{H(x)-\hat{H}(x)}{\lambda}\right) d x \\
+\int_{B(\delta)} x^{\theta-1} \cdot \exp \left(\frac{\hat{H}(x)}{\lambda}\right) \cdot S_{M^{\prime}}\left(\frac{H(x)-\hat{H}(x)}{\lambda}\right) d x
\end{aligned}
$$


where :

$$
\left|S_{M^{\prime}}\left(\frac{H(x)-\hat{H}(x)}{\lambda}\right)\right| \leq\left|\left(\frac{H(x)-\hat{H}(x)}{\lambda}\right)\right|^{M^{\prime}+1} \exp \left|\left(\frac{H(x)-\hat{H}(x)}{\lambda}\right)\right|
$$

1) Let us first show that the second term in the right-hand side of (4) is $o\left(\lambda^{M}\right)$.

For $r \geq 1, x \in \mathbb{R}^{p}$, let $\epsilon_{r}(x)=H(x)-\sum_{1 \leq A(\mu) \leq r} a_{\mu} x^{\mu}$.

$$
H(x)=\sum_{A(\mu)=1} a_{\mu} x^{\mu}+\epsilon_{1}(x)=\hat{H}(x)+\epsilon_{1}(x)
$$

We have assumed that $H \neq \hat{H}$, so $\Sigma(H) \backslash \Delta(H) \neq \emptyset$.

Set $\eta_{0}=\inf \left(A_{\Delta}(\mu)-1 ; \mu \in \Sigma(H) \backslash \Delta(H)\right)$. $\eta_{0}$ is clearly positive.

There exists no $\mu$ in $\Sigma(H)=\left\{\mu \in \mathbb{N}^{p} ; a_{\mu} \neq 0\right\}$ strictly between $\Delta$ and $\left(1+\eta_{0}\right) . \Delta$ , i.e. satisfying $1<A(\mu)<1+\eta_{0}$.

Then for $x \in B(\delta),\left|\epsilon_{1}(x)\right| \leq$ const. $\sum_{A(\mu)=1}|x|^{\left(1+\eta_{0}\right) \mu} \leq$ const. $\sum_{\mu \in S_{\Delta}}|x|^{\left(1+\eta_{0}\right) \mu}$

$$
\begin{aligned}
|(H-\hat{H})(x)|=\left|\epsilon_{1}(x)\right| & \leq \text { const. } \sum_{\mu \in S_{\Delta}}|x|^{\left(1+\eta_{0}\right) \mu} \\
& \leq \text { const. }\left(\sum_{\mu \in S_{\Delta}} x^{\mu}\right)^{1+\eta_{0}} \leq \text { const. }|\hat{H}(x)|^{1+\eta_{0}}
\end{aligned}
$$

by Lemma 3 since $\hat{H}$ is assumed to be non degenerate (and every vertex has even coordinates). From this it follows that :

$$
\begin{gathered}
\left|S_{M^{\prime}}\left(\frac{H(x)-\hat{H}(x)}{\lambda}\right)\right| \leq\left|\left(\frac{H(x)-\hat{H}(x)}{\lambda}\right)\right|^{M^{\prime}+1} \exp \left|\left(\frac{H(x)-\hat{H}(x)}{\lambda}\right)\right| \\
\quad \leq \text { const. } \lambda^{-M^{\prime}-1} \cdot\left(\sum_{\mu \in S_{\Delta}}\left|x^{\mu}\right|\right)^{\left(1+\eta_{0}\right)\left(M^{\prime}+1\right)} \cdot \exp \left(\frac{1}{\lambda} \text { const. }|\hat{H}(x)|^{1+\eta_{0}}\right)
\end{gathered}
$$

then for $\delta>0$ small enough :

$$
\begin{aligned}
& \qquad\left|\int_{B(\delta)} x^{\theta-1} \cdot \exp \left(\frac{\hat{H}(x)}{\lambda}\right) \cdot S_{M^{\prime}}\left(\frac{H(x)-\hat{H}(x)}{\lambda}\right) d x\right| \\
& \leq \text { const. } \frac{1}{\lambda^{M^{\prime}+1}} \sum_{\mu \in S_{\Delta}}\left|\int_{B(\delta)}\right| x^{\theta-1}|\cdot| x^{\mu\left(1+\eta_{0}\right)\left(M^{\prime}+1\right)}\left|\cdot \exp \left(\frac{1}{2 \lambda} \cdot \hat{H}(x)\right) d x\right|
\end{aligned}
$$

The powers of $x$ are here: $\theta-1+\left(1+\eta_{0}\right)\left(M^{\prime}+1\right) \mu$, for $\mu \in S \subset \Delta$. Each integral has the index:

$$
\begin{aligned}
& A_{\Delta}\left(\theta+\left(1+\eta_{0}\right)\left(M^{\prime}+1\right) \mu\right) \geq A_{\Delta}(\theta)+\left(1+\eta_{0}\right)\left(M^{\prime}+1\right) A_{\Delta}(\mu) \\
= & A_{\Delta}(\theta)+\left(1+\eta_{0}\right)\left(M^{\prime}+1\right)
\end{aligned}
$$


According to Lemma 4, the integral on the left hand side of $(5)$ is $o\left(\lambda^{M}\right)$ if

$$
A_{\Delta}(\theta)+\left(1+\eta_{0}\right)\left(M^{\prime}+1\right)-\left(M^{\prime}+1\right)>M
$$

i.e. if

$$
M^{\prime}+1>\frac{M-A_{\Delta}(\theta)}{\eta_{0}}
$$

which is true if $M^{\prime}$ is the integer part of $\frac{M-A_{\Delta}(\theta)}{\eta_{0}}$.

2) Set

$$
R_{k}(x)=\frac{1}{k !}\left[\sum_{1<A(\mu) \leq M+1} a_{\mu} x^{\mu}\right]^{k}
$$

We want now to prove that these polynomials satisfy

$$
\begin{aligned}
& \frac{1}{k !} \int_{B(\delta)} x^{\theta-1} \cdot \exp \left(\frac{\hat{H}(x)}{\lambda}\right) \cdot\left(\frac{H(x)-\hat{H}(x)}{\lambda}\right)^{k} d x \\
= & o\left(\lambda^{M}\right)+\frac{1}{\lambda^{k}} \int_{B(\delta)} x^{\theta-1} R_{k}(x) \exp \left(\frac{\hat{H}(x)}{\lambda}\right) d x
\end{aligned}
$$

We know that

$$
\begin{aligned}
\frac{H(x)-\hat{H}(x)}{\lambda}=\frac{1}{\lambda}\left(\sum_{1<A(\mu) \leq M+1} a_{\mu} x^{\mu}+\epsilon_{M+1}(x)\right) \\
\left(\frac{H(x)-\hat{H}(x)}{\lambda}\right)^{k}=\frac{1}{\lambda^{k}}\left(\sum_{1<A(\mu) \leq M+1} a_{\mu} x^{\mu}\right)^{k} \\
+\frac{1}{\lambda^{k}} \sum_{i=1}^{k}\left(\begin{array}{c}
k \\
i
\end{array}\right)\left(\sum_{1<A(\mu) \leq M+1} a_{\mu} x^{\mu}\right)^{k-i}\left(\epsilon_{M+1}(x)\right)^{i}
\end{aligned}
$$

Let us show that the contribution of the second term is $o\left(\lambda^{M}\right)$.

It is enough to prove that

$$
\frac{1}{\lambda^{k}} \int_{B(\delta)} x^{\theta-1} x^{\nu(1)+. .+\nu(k-i)}\left(\epsilon_{M+1}(x)\right)^{i} \exp \left(\frac{\hat{H}(x)}{\lambda}\right) d x
$$

is $o\left(\lambda^{M}\right)$ as $\left(\lambda \rightarrow 0^{+}\right)$, where here $A(\nu(j))>1$ for $1 \leq j \leq k-i$.

We know that $\left|\epsilon_{M+1}(x)\right|^{i} \leq$ const. $\sum_{\mu^{\prime} \in S_{\Delta}}\left|x^{(M+1) \mu^{\prime}}\right|^{i}$ thus the index of the integral in $(7)$ is not lower than

$$
\begin{gathered}
A\left(\theta+(M+1) \mu^{\prime} . i+\nu(1)+. .+\nu(k-i)\right) \geq A(\theta)+(M+1) i+k-i \\
=A(\theta)+M i+k \geq A(\theta)+M+k>M+k
\end{gathered}
$$


which gives that (7) is $o\left(\lambda^{M+k}\right) \cdot \lambda^{-k}=o\left(\lambda^{M}\right)$ as $\lambda \rightarrow 0^{+}$

3) We prove now (1).

For $k \geq 1$,

$$
\begin{aligned}
& \frac{1}{\lambda^{k}} \int_{B(\delta)} x^{\theta-1} R_{k}(x) \exp \left(\frac{\hat{H}(x)}{\lambda}\right) d x \\
= & \frac{1}{\lambda^{k}} \sum_{\nu(1), . . \nu(k)} \text { const } \int_{B(\delta)} x^{\theta-1} x^{\nu(1)+. .+\nu(k)} \exp \left(\frac{\hat{H}(x)}{\lambda}\right) d x
\end{aligned}
$$

where the $\nu(j) \in \Sigma(H)$ are such that $\nu(j)>1$, i.e. $\nu(j) \geq 1+\eta_{0}$.

The index of this last integral is $A_{\Delta}\left(\theta+\sum \nu(i)\right) \geq A_{\Delta}(\theta)+\sum_{1}^{k} A_{\Delta}(\nu(i)) \geq A_{\Delta}(\theta)+k\left(1+\eta_{0}\right)$

According to Lemma 4 (ii), we have then, for any $\varepsilon>0$, $\frac{1}{\lambda^{k}} \int_{B(\delta)} x^{\theta-1} R_{k}(x) \exp \left(\frac{\hat{H}(x)}{\lambda}\right) d x=o\left(\lambda^{A_{\Delta}(\theta)+k\left(1+\eta_{0}\right)-\varepsilon}\right) \cdot \lambda^{-k}$

$$
=o\left(\lambda^{A_{\Delta}(\theta)+k \eta_{0}-\varepsilon}\right)
$$

3.6. Proof of Proposition 4. Let $\theta \in \mathbb{N}^{* p}$. There exists $c>0$, such that

$$
\begin{aligned}
\int_{\|x\| \geq \delta}\left|x^{\theta-1}\right| \cdot \exp \left(\frac{\hat{H}(x)}{\lambda}\right) d x & \leq \int_{\|x\| \geq \delta}\left|x^{\theta-1}\right| \cdot \exp \left(\frac{-c}{\lambda} \cdot \sum_{\mu \in S_{\Delta}} x^{\mu}\right) d x \\
& =2^{p} \int_{\|x\| \geq \delta, x \in \mathbb{R}_{+}^{p}} x^{\theta-1} \cdot \exp \left(\frac{-c}{\lambda} \cdot \sum_{\mu \in S_{\Delta}} x^{\mu}\right) d x
\end{aligned}
$$

from Lemma 3, because $\hat{H}$ is supposed to be non degenerate.

Let us show thus that $K(\theta)=\int_{\|x\| \geq \delta, x \in \mathbb{R}_{+}^{p}} x^{\theta-1} \cdot \exp \left(\frac{-c}{\lambda} \cdot \sum_{\mu \in S_{\Delta}} x^{\mu}\right) d x$ is $O\left(e^{-d / \lambda}\right)$.

Since $H$ is convenient, there exist $p$ vertices $\mu(1), \ldots, \mu(p)$ of $\Delta$ on the coordinate axes: $\mu(1)=\left(D_{1}, 0, . ., 0\right), . ., \mu(p)=\left(0, . ., 0, D_{p}\right)$, where the $D_{i} \in \mathbb{N}^{*}$.

$$
\begin{aligned}
0 & \leq K(\theta) \leq \int_{\|x\| \geq \delta, x \in \mathbb{R}_{+}^{p}} x^{\theta-1} \cdot \exp \left(\frac{-c}{\lambda} \cdot \sum_{i=1}^{p} x_{i}^{D_{i}}\right) d x \\
& \leq \sum_{j=1}^{p} \int_{x_{j}^{D_{j}} \geq \delta_{2}, x \in \mathbb{R}_{+}^{p}} x^{\theta-1} \cdot \exp \left(\frac{-c}{\lambda} \cdot \sum_{i=1}^{p} x_{i}^{D_{i}}\right) d x
\end{aligned}
$$

for $\delta_{2}>0$ small enough, because for $\delta_{2}$ small enough, $\left|x_{j}^{D_{j}}\right|<\delta_{2}$ for each $j$ implies $\|x\|<\delta$. 
Setting $u_{i}=\lambda^{-\frac{1}{D_{i}}} x_{i}, x_{i}^{D_{i}}=\lambda \cdot u_{i}^{D_{i}}$

$$
\begin{gathered}
K(\theta) \leq \sum_{j=1}^{p} \int_{\lambda u_{j}^{D_{j}} \geq \delta_{2}, u \in \mathbb{R}_{+}^{p}} \lambda^{\sum_{i} \frac{\theta_{i}}{D_{i}}} \exp \left(-c \cdot \sum_{i=1}^{p} u_{i}^{D_{i}}\right) \cdot u^{\theta-1} d u \\
=\lambda^{\sum_{i} \frac{\theta_{i}}{D_{i}}} \sum_{j=1}^{p} \int_{\lambda u_{j}^{D_{j}} \geq \delta_{2}} \exp \left(-c \cdot u_{j}^{D_{j}}\right) u_{j}^{\theta_{j}-1} d u_{j} \cdot \prod_{i \neq j} \int_{0}^{+\infty} \exp \left(-c \cdot u_{i}^{D_{i}}\right) \cdot u_{i}^{\theta_{i}-1} d u_{i} \\
\leq \lambda^{\sum_{i} \frac{\theta_{i}}{D_{i}}} \sum_{j=1}^{p} \int_{\lambda u_{j}^{D_{j}} \geq \delta_{2}} \exp \left(\frac{-c}{2} \cdot u_{j}^{D_{j}}\right) \exp \left(\frac{-c}{2 \lambda} \cdot \delta_{2}\right) \cdot u_{j}^{\theta_{j}-1} d u_{j} \\
\cdot \prod_{i \neq j} \int_{0}^{+\infty} \exp \left(-c \cdot u_{i}^{D_{i}}\right) \cdot u_{i}^{\theta_{i}-1} d u_{i} \\
K(\theta) \leq \lambda^{\sum_{i} \frac{\theta_{i}}{D_{i}}} \exp \left(\frac{-c}{2 \lambda} \cdot \delta_{2}\right) \sum_{j=1}^{p} \int_{0}^{+\infty} \exp \left(\frac{-c}{2} \cdot u_{j}^{D_{j}}\right) \cdot u_{j}^{\theta_{j}-1} d u_{j} \\
\cdot \prod_{i \neq j} \int_{0}^{+\infty} \exp \left(-c \cdot u_{i}^{D_{i}}\right) \cdot u_{i}^{\theta_{i}-1} d u_{i}
\end{gathered}
$$

Each integral is convergent, because $\theta_{i} \geq 1$ for each $i$, since $\theta \in \mathbb{N}^{* p}$. We thus obtain $K(\theta)=O\left(e^{-d / \lambda}\right) \quad$ as $\lambda \rightarrow 0^{+}$with $\left.d \in\right] 0 ; \frac{c}{2} \delta_{2}[$.

3.7. Proof of Proposition 5. We consider

$$
\int_{\mathbb{R}^{p}} \exp \left(\frac{H_{B \cup S}(x)}{\lambda}\right) \cdot x^{\theta-1} d x
$$

where $H_{B \cup S}(x)=\sum_{\mu \in B \cup S} a_{\mu} x^{\mu}$, with $B \cap S=\emptyset, B$ is included in $m$ facets $F_{1}, \ldots$, $F_{m}$ and not less. We denote by $\Delta_{i}$ the set of the elements of $B$ which are only in the facet $F_{i}$, i.e. $\Delta_{i}=B \cap\left(F_{i} \backslash \cup_{j \neq i} F_{j}\right), i=1 ; 2$. Since $B$ is not included in less that $m$ facets, $\Delta_{1}$ and $\Delta_{2}$ are non empty. Moreover we have $\Delta_{1 \cap} \Delta_{2}=\emptyset$.

Let us define

$$
B_{1}=B \backslash \Delta_{1}, B_{2}=B \backslash \Delta_{2}, B_{0}=B \backslash\left(\Delta_{1} \cup \Delta_{2}\right)
$$

We have

$$
\int_{\mathbb{R}^{p}} \exp \left(\frac{H_{B \cup S}(x)}{\lambda}\right) \cdot x^{\theta-1} d x=\sum_{k_{1}=0}^{\infty} \sum_{k_{2}=0}^{\infty} \hat{L}_{k_{1}, k_{2}}
$$

where

$$
\begin{aligned}
\hat{L}_{k_{1}, k_{2}}= & \frac{1}{k_{1} ! k_{2} !} \frac{1}{\lambda^{k_{1}+k_{2}}} \cdot \int_{\mathbb{R}^{p}}\left(\sum_{\mu \in \Delta_{1}} a_{\mu} x^{\mu}\right)^{k_{1}}\left(\sum_{\mu \in \Delta_{2}} a_{\mu} x^{\mu}\right)^{k_{2}} \exp \left(\frac{H_{S \cup B_{0}}(x)}{\lambda}\right) \cdot x^{\theta-1} d x \\
= & \frac{1}{k_{1} ! k_{2} !} \frac{1}{\lambda^{k_{1}+k_{2}}} \sum_{\nu(1), ., \nu\left(k_{1}\right) \in \Delta_{1}} a_{\nu(1)} \ldots a_{\nu\left(k_{1}\right)} \\
& \cdot\left[\sum_{\nu^{\prime}(1), . ., \nu^{\prime}\left(k_{2}\right) \in \Delta_{2}} a_{\nu^{\prime}(1)} \ldots a_{\nu^{\prime}\left(k_{2}\right)} \int_{\mathbb{R}^{p}} \exp \left(\frac{H_{S \cup B_{0}}(x)}{\lambda}\right) \cdot x^{\theta-1} x^{\sum \nu(l)+\sum \nu^{\prime}(l)} d x\right]
\end{aligned}
$$


First, we want to show that if $k_{1}>M$ " and $k_{2}>M$ ", with $M$ " large enough, then $\hat{L}_{k_{1}, k_{2}}=o\left(\lambda^{M}\right)$.

Recall that the index of the integral (see the definition after Lemma 4) gives the order of magnitude of this integral.

The index of

$$
\int_{\mathbb{R}^{p}} \exp \left(\frac{H_{S \cup B_{0}}(x)}{\lambda}\right) \cdot x^{\theta-1} x^{\sum \nu(l)+\sum \nu^{\prime}(l)} d x
$$

is

$$
\begin{aligned}
\inf _{F} A_{F}\left(\sum \nu(l)+\sum \nu^{\prime}(l)+\theta\right) & =\inf _{F}\left[A_{F}\left(\sum \nu(l)\right)+A_{F}\left(\sum \nu^{\prime}(l)\right)+A_{F}(\theta)\right] \\
& \geq \inf _{F} T_{F}+A_{\Delta}(\theta)
\end{aligned}
$$

where

$$
T_{F}=\left[\sum_{l=1}^{k_{1}} A_{F}(\nu(l))+\sum_{l=1}^{k_{2}} A_{F}\left(\nu^{\prime}(l)\right)\right]
$$

For any facet $F, A_{F}(\nu(l)) \geq 1$ and $A_{F}\left(\nu^{\prime}(l)\right) \geq 1$ thus $T_{F} \geq k_{1}+k_{2}$

By construction of the $\Delta_{i}$, we have $\Delta_{i} \cap F_{j}=\emptyset$ if $j \neq i$.

Let $\eta_{i}=\inf \left(A_{F_{j}}(\mu)-1 ; \mu \in \Delta_{i}\right.$ and $\left.j \neq i\right), i=1 ; 2$.

For any $l \in\left[1 ; k_{1}\right], A_{F}(\nu(l)) \geq 1+\eta_{1}$ if $F \neq F_{1}$

For any $l \in\left[1 ; k_{2}\right], A_{F}\left(\nu^{\prime}(l)\right) \geq 1+\eta_{2}$ if $F \neq F_{2}$

Hence $T_{F} \geq k_{1}\left(1+\eta_{1}\right)+k_{2}$ if $F \neq F_{1}$

and $T_{F} \geq k_{1}+k_{2}\left(1+\eta_{2}\right)$ if $F \neq F_{2}$

So for any facet $F, T_{F} \geq k_{1}+k_{2}+\eta \inf \left(k_{1}, k_{2}\right)>0$, where $\eta=\inf \left(\eta_{1}, \eta_{2}\right)$. This implies that the index of (8) is greater or equal to $k_{1}+k_{2}+\eta \inf \left(k_{1}, k_{2}\right)+A_{\Delta}(\theta)$.

Hence, according to Lemma 4 , we have $\hat{L}_{k_{1}, k_{2}}=o\left(\lambda^{M}\right)$ if $\eta \inf \left(k_{1}, k_{2}\right)+A_{\Delta}(\theta)>$ $M$, i.e. if $\inf \left(k_{1}, k_{2}\right)>\frac{1}{\eta}\left(M-A_{\Delta}(\theta)\right)$. It is then sufficient to take $M$ " the integer part of $\frac{1}{\eta}\left(M-A_{\Delta}(\theta)\right)$.

We can write then

$$
\begin{aligned}
& \int_{\mathbb{R}^{p}} \exp \left(\frac{H_{S \cup B}(x)}{\lambda}\right) \cdot x^{\theta-1} d x \\
= & o\left(\lambda^{M}\right)+\sum_{k_{1}=0}^{M \prime} \sum_{k_{2}=0}^{\infty} \hat{L}_{k_{1}, k_{2}}+\sum_{k_{1}=0}^{\infty} \sum_{k_{2}=0}^{M^{\prime \prime}} \hat{L}_{k_{1}, k_{2}}-\sum_{k_{1}=0}^{M \prime} \sum_{k_{2}=0}^{M^{\prime \prime}} \hat{L}_{k_{1}, k_{2}} \\
= & o\left(\lambda^{M}\right)+\sum_{k_{1}=0}^{M^{\prime \prime}} \frac{1}{k_{1} ! \lambda^{k_{1}}} \int_{\mathbb{R}^{p}}\left(\sum_{\mu \in \Delta_{1}} a_{\mu} x^{\mu}\right)^{k_{2}} \exp \left(\frac{H_{S \cup B_{1}}(x)}{\lambda}\right) \cdot x^{\theta-1} d x \\
& +\sum_{k_{2}=0}^{M^{\prime \prime}} \frac{1}{k_{2} ! \lambda^{k_{2}}} \int_{\mathbb{R}^{p}}\left(\sum_{\mu \in \Delta_{2}} a_{\mu} x^{\mu}\right)^{k_{2}} \exp \left(\frac{H_{S \cup B_{2}}(x)}{\lambda}\right) \cdot x^{\theta-1} d x \\
& -\sum_{k_{1}=0}^{M^{\prime \prime}} \sum_{k_{2}=0}^{M^{\prime \prime}} \frac{1}{k_{1} ! k_{2} ! \lambda^{k_{1}+k_{2}}} \int_{\mathbb{R}^{p}}\left(\sum_{\mu \in \Delta_{1}} a_{\mu} x^{\mu}\right)^{k_{1}} \\
& \left(\sum_{\mu \in \Delta_{2}} a_{\mu} x^{\mu}\right)^{k_{2}} \exp \left(\frac{H_{S \cup B_{0}}(x)}{\lambda}\right) \cdot x^{\theta-1} d x .
\end{aligned}
$$




\section{Proof of Proposition 6.}

4.1. First step of the proof of Proposition 6. We will show in this first step that we just have to study $M_{B}$ given in (9), and we will state Lemma 7 which will be useful next.

We study

$$
L\left(b^{\prime}, b ", P\right)=\int_{\mathbb{R}_{+}^{2}} \exp \left(\frac{P(x)}{\lambda}\right) \cdot x^{b^{\prime}-1}(\ln x)^{b^{\prime \prime}} d x
$$

where $P(x)=\sum_{\mu \in F \cup S} a_{\mu} x^{\mu},\left(b^{\prime}, b^{\prime \prime}, P\right)$ satisfying the HP hypothesis.

$F$ is a given facet of $\Delta, S$ is the set of the vertices of the Newton diagram $\Delta$ of $P$. $b^{\prime}$ is in the interior of $\mathcal{C}(\Delta), b " \in \mathbb{N}^{2}$.

Since $\nu$ is a bordering vertex of $\Delta$, one can find a facet-system $\sigma=(\mu(1), \mu(2))$ of the 2 vertices of a same facet of $\Delta$ such that $\nu$ is on one side (strictly) of $(O, \mu(1))$ and the other points of the support $\Sigma(P)$ of $P$ are on the other side. One makes then the change of variables $x \rightarrow v^{(\sigma)}$ (defined in Section 3.2.1):

$$
L\left(b^{\prime}, b ", P\right)=\int_{\mathbb{R}_{+}^{2}} \exp \left(\frac{1}{\lambda} \sum_{\mu \in \Delta} a_{\mu} v^{\beta(\mu)}\right) v^{b-1} \prod_{i=1}^{2}\left(\ln \left(v^{\beta\left(e_{i}\right)}\right)\right)^{b^{\prime \prime}{ }_{i}} . D \cdot d v
$$

with $b=\beta\left(b^{\prime}\right)$, where $e_{1}=(1 ; 0)$, and $e_{2}=(0 ; 1)$, and

$$
D \prod_{i=1}^{2}\left(\ln \left(v^{\beta\left(e_{i}\right)}\right)\right)^{b^{\prime \prime}{ }_{i}}=D \prod_{i=1}^{2}\left(\sum_{j=1}^{2} \beta_{j}\left(e_{i}\right) \ln \left(v_{j}\right)\right)^{b^{\prime \prime}{ }_{i}}=\sum_{|B|=\left|b^{\prime \prime}\right|} b_{B}(\ln v)^{B}
$$

where $B \in \mathbb{N}^{2},|B|=B_{1}+B_{2},|b "|=b^{\prime \prime}{ }_{1}+b^{\prime \prime}{ }_{2},(\ln v)^{B}=\prod_{i=1}^{2}\left(\ln v_{i}\right)^{B_{i}}$, the $b_{B}$ are constants.

$$
L\left(b^{\prime}, b^{\prime \prime}, P\right)=\sum_{|B|=\left|b^{\prime \prime}\right|} b_{B} M_{B}
$$

setting

$$
M_{B}=\int_{\mathbb{R}_{+}^{2}} \exp \left(\frac{1}{\lambda} \sum_{\mu \in \Delta} a_{\mu} v^{\beta(\mu)}\right) v^{b-1}(\ln v)^{B} d v
$$

It is then enough to study $M_{B}$. Our aim is now to remove the vertex $\nu$ of $\Delta$, in $M_{B}$.

We split the integrand in two parts to put it on the form of a product $\phi . f$ which will be integrated (with respect to $v_{2}$ ) according to Lemma 7 (given further). $\nu$ is the vertex which will disappear. $\mu(2)$ and $\nu$ are on both sides of $(O, \mu(1))$ then, with perhaps a new order of the $\mu(i)$ :

$v^{\beta(\nu)}=v_{1}^{\beta_{1}(\nu)} v_{2}^{\beta_{2}(\nu)}$ where $\beta_{1}(\nu)>0, \beta_{2}(\nu)<0$.

We introduce new notations:

$$
t=v_{2} \quad, \quad w=v_{1} \quad \text { and } \quad k=B_{2}
$$

So

$$
\begin{aligned}
M_{B}= & \int_{\mathbb{R}_{+}^{2}} \exp \left(\frac{1}{\lambda} \sum_{\mu \in \Delta \backslash\{\nu\}} a_{\mu} w^{\beta_{1}(\mu)} t^{\beta_{2}(\mu)}\right) w^{b_{1}-1} \cdot t^{b_{2}-1}(\ln w)^{B_{1}} \\
& (\ln t)^{k} \cdot \exp \left(\frac{1}{\lambda} a_{\nu} w^{\beta_{1}(\nu)} t^{\beta_{2}(\nu)}\right) d w d t
\end{aligned}
$$


There exist $c \in \mathbf{Z}$ and $\alpha \in] 0 ; 1]$ (uniquely defined) such that:

$$
\begin{gathered}
b_{2}-1=c-\alpha \\
M_{B}=\int_{\mathbb{R}_{+}^{2}} \exp \left(\frac{1}{\lambda} \sum_{\mu \in \Delta \backslash\{\nu\}} a_{\mu} w^{\beta_{1}(\mu)} t^{\beta_{2}(\mu)}\right) w^{b_{1}-1} \cdot t^{c^{+}}(\ln w)^{B_{1}} \\
(\ln t)^{k} \cdot \exp \left(\frac{1}{\lambda} a_{\nu} w^{\beta_{1}(\nu)} t^{\beta_{2}(\nu)}\right) \frac{1}{t^{\alpha+c^{-}}} d w d t
\end{gathered}
$$

where $c^{+}=\sup (c, 0)$ and $c^{-}=\sup (-c, 0)$

Now, to complete the proof of Proposition 6 , we wish to remove the second exponential in $M_{B}$, but expanding it directly on the form $e^{x}=\sum_{k \geq 0} \frac{x^{k}}{k !}$ is not possible because of the $t$ at the denominator (since $\beta_{2}(\nu)<0$ ).

We will use two lemmas given in Wong [14] (Lemmas 1 and 2 pages 296 and 297), that we sum up in Lemma 6.

If $f(t)$ is a locally integrable function on $\left[0 ;+\infty\left[\right.\right.$, with $f(t) \sim \sum_{s=0}^{\infty} \frac{a_{s}}{t^{s+\alpha}}$ as $t \rightarrow+\infty$, where $\alpha \in] 0 ; 1]$, we want to compute $\int_{0}^{+\infty} f(t) . \phi(t) d t$ for $\phi \in \mathcal{S}$, in terms of the asymptotic expansion of $f$. For us here, $f$ will be essentially the second exponential in (12) and $\phi$ will be the first (for $w$ stated). Recall that $\mathcal{S}$ is the space of rapidly decreasing functions i.e.

$$
\phi \in \mathcal{S} \Leftrightarrow \phi \in C^{\infty}(\mathbb{R}, \mathbb{R}), \forall(p, q) \in \mathbb{N}^{2}, \exists c_{p, q}>0, \forall x \in \mathbb{R},\left|x^{p} \phi^{(q)}(x)\right| \leq c_{p, q}
$$

Let

$$
\begin{gathered}
f_{n}(t)=f(t)-\frac{a_{0}}{t^{\alpha}}-. .-\frac{a_{n-1}}{t^{n-1+\alpha}} \quad \text { and } \quad f_{n, 0}=f_{n} \\
f_{n, j+1}(t)=-\int_{t}^{+\infty} f_{n, j}(\tau) d \tau=\frac{(-1)^{j+1}}{j !} \cdot \int_{t}^{\infty}(\tau-t)^{j} f_{n}(\tau) d \tau
\end{gathered}
$$

In particular,

$$
f_{n, n}(t)=\frac{(-1)^{n}}{(n-1) !} \cdot \int_{t}^{\infty}(\tau-t)^{n-1} f_{n}(\tau) d \tau
$$

We can now define the following distributions:

$$
\begin{aligned}
& <f ; \phi>=\int_{0}^{+\infty} f(t) \phi(t) d t \quad \text { and } \quad<\delta^{(s-1)} ; \phi>=(-1)^{s-1} \phi^{(s-1)}(0) \\
& <f_{n} ; \phi>=(-1)^{n} \int_{0}^{+\infty} f_{n, n}(t) \phi^{(n)}(t) d t
\end{aligned}
$$

- For $0<\alpha<1,<t_{+}^{-\alpha} ; \phi>=\int_{0}^{\infty} \frac{\phi(t)}{t^{\alpha}} d t$ for $\phi \in \mathcal{S}$.

Since $D^{s}\left(t^{-\alpha}\right) \cdot \frac{(-1)^{s}}{(\alpha)_{s}}=t^{-s-\alpha}$ with $(\alpha)_{s}=\alpha(\alpha+1) . .(\alpha+s-1)$ (ordinary derivative), we can define the distribution $t_{+}^{-s-\alpha}$ as $t_{+}^{-s-\alpha}=D^{s}\left(t_{+}^{-\alpha}\right) \cdot \frac{(-1)^{s}}{(\alpha)_{s}}$ (distributional derivative), i.e.

$$
<t_{+}^{-s-\alpha} ; \phi>=\frac{1}{(\alpha)_{s}} \int_{0}^{\infty} \frac{\phi^{(s)}(t)}{t^{\alpha}} d t
$$


- For $\alpha=1,<\ln t_{+} ; \phi>=\int_{0}^{\infty} \ln t . \phi(t) d t$ for $\phi \in \mathcal{S}$. similarly since $D^{s+1}(\ln t) \cdot \frac{(-1)^{s}}{s !}=t^{-s-1}$, we define the distribution $t_{+}^{-s-1}$ as $t_{+}^{-s-1}=$ $D^{s+1}\left(\ln _{+} t\right) \cdot \frac{(-1)^{s}}{s !}$ i.e.

$$
<t_{+}^{-s-1} ; \phi>=\frac{-1}{s !} \int_{0}^{\infty}(\ln t) \cdot \phi^{(s+1)}(t) d t
$$

Lemma 6. Let $f$ be a locally integrable function on $[0 ;+\infty[$.

If $f(t) \sim \sum_{s=0}^{\infty} \frac{a_{s}}{t^{s+\alpha}} \quad$ as $\left.\left.t \rightarrow+\infty, \alpha \in\right] 0 ; 1\right]$,

then for any $\phi \in \mathcal{S}$, and $n \in \mathbb{N}^{*}$ :

$$
<f, \phi>=\sum_{s=0}^{n-1} a_{s}<t_{+}^{-s-\alpha} ; \phi>-\sum_{s=1}^{n} c_{s}<\delta^{(s-1)} ; \phi>+<f_{n} ; \phi>
$$

- If $0<\alpha<1, c_{s}=f_{s, s}(0)$

- If $\alpha=1$, then $c_{s}=\lim _{t \rightarrow 0}\left(f_{s, s}(t)+\frac{(-1)^{s-1}}{(s-1) !} a_{s-1} \ln t\right)$

Proof. See Lemmas 1 and 2 of Wong [14], pages 296-298.

Our aim is now to generalize Lemma 6 to a function $f$ satisfying $f(t) \sim \sum_{s=0}^{\infty} \frac{a_{s}}{t^{s+\alpha}}(\ln t)^{k} \quad$ as $t \rightarrow+\infty$, where $k \in \mathbb{N}$

Here

$$
f_{n}(t)=f(t)-\left[\frac{a_{0}}{t^{\alpha}}+\ldots+\frac{a_{n-1}}{t^{n-1+\alpha}}\right](\ln t)^{k}
$$

Let $F_{k, s, \alpha}(t)$ be the function defined for $0<\alpha<1$ by :

$$
\begin{aligned}
F_{k, s, \alpha}(t)= & \frac{(-1)^{s}}{(\alpha)_{s} t^{\alpha}} \cdot\left[(\ln t)^{k}+k(\ln t)^{k-1}\left(\sum_{l=1}^{s} \frac{1}{s-l+\alpha}\right)\right. \\
& \left.+. .+k ! \sum_{1 \leq l_{1} \leq . . \leq l_{k} \leq s} \frac{1}{\left(s-l_{1}+\alpha\right) \ldots\left(s-l_{k}+\alpha\right)}\right] \\
= & (-1)^{s} \sum_{r=0}^{k} K(k, s, \alpha, r) \frac{(\ln t)^{r}}{t^{\alpha}}
\end{aligned}
$$

where

$$
K(k, s, \alpha, r)=\sum_{1 \leq l_{1} \leq . . \leq l_{k-r} \leq s} \frac{1}{\left(s-l_{1}+\alpha\right) . .\left(s-l_{k-r}+\alpha\right)} \cdot \frac{k !}{r !(\alpha)_{s}}
$$

An easy calculation shows that $D^{s}\left(F_{k, s, \alpha}\right)(t)=\frac{(\ln t)^{k}}{t^{s+\alpha}}$ (ordinary derivative), so as $t_{+}^{-s-\alpha}$ has been defined by $t_{+}^{-s-\alpha}=D^{s}\left(t_{+}^{-\alpha}\right) \cdot \frac{(-1)^{s}}{(\alpha)_{s}}$ (distributional derivative), one can define the distribution $(\ln t)_{+}^{k} t_{+}^{-s-\alpha}=D^{s}\left(F_{k, s, \alpha}(t)\right)$, for $\left.\alpha \in\right] 0 ; 1[$, i.e. for any $\phi \in \mathcal{S}$ :

$$
<(\ln t)_{+}^{k} t_{+}^{-s-\alpha} ; \phi>=(-1)^{s} \int_{0}^{\infty} \phi^{(s)}(t) F_{k, s, \alpha}(t) d t
$$


In the same way :

$$
\begin{aligned}
F_{k, s, 1}(t)= & \frac{(-1)^{s}}{s !} \cdot\left[\frac{(\ln t)^{k+1}}{k+1}+(\ln t)^{k}\left(\sum_{l=1}^{s} \frac{1}{s-l+1}\right)+. .\right. \\
& \left.+(\ln t) . k ! \sum_{1 \leq l_{1} \leq . . \leq l_{k} \leq s} \frac{1}{\left(s-l_{1}+1\right) \ldots\left(s-l_{k}+1\right)}\right] \\
= & (-1)^{s} \sum_{r=1}^{k+1} K(k, s, 1, r) .(\ln t)^{r}
\end{aligned}
$$

From $D^{s+1}\left(F_{k, s, 1}\right)(t)=\frac{(\ln t)^{k}}{t^{s+1}}$, we denote by $(\ln t)_{+}^{k} t_{+}^{-s-1}$ the $(s+1)^{t h}$ distributional derivative of the distribution on $\left[0 ;+\infty\left[\right.\right.$ corresponding to $F_{k, s, 1}$. We can now state the next lemma, which generalizes that of Wong.

LEMMA 7. Let $f$ be a locally integrable function on $[0 ;+\infty[$.

If $f(t) \sim \sum_{s=0}^{\infty} \frac{a_{s}}{t^{s+\alpha}}(\ln t)^{k} \quad$ as $\left.\left.t \rightarrow+\infty, \alpha \in\right] 0 ; 1\right], k \in \mathbb{N}$ then for any $\phi \in \mathcal{S}$, and $n \in \mathbb{N}^{*}$ :

$$
<f, \phi>=\sum_{s=0}^{n-1} a_{s}<(\ln t)_{+}^{k} t_{+}^{-s-\alpha} ; \phi>-\sum_{s=1}^{n} c_{s}<\delta^{(s-1)} ; \phi>+<f_{n} ; \phi>
$$

- If $0<\alpha<1, c_{s}=f_{s, s}(0)$

- If $\alpha=1$, then $c_{s}=\lim _{t \rightarrow 0}\left(f_{s, s}(t)+a_{s-1} F_{k ; s-1 ; 1}(t)\right)$

Proof of Lemma \%. We adapt the proof of Lemma 1 and 2 of Wong ([14], p296). 1) Case $0<\alpha<1$.

The question is to extend Lemma 6 to functions $f$ satisfying, for $0<\alpha<1, f(t) \sim$ $\sum_{s=0}^{\infty} \frac{a_{s}(\ln t)^{k}}{t^{s+\alpha}}$, as $t \rightarrow+\infty$.

We define $F_{k, s, \alpha}$ as in (15). $(\ln t)_{+}^{k} t_{+}^{-s-\alpha}$ will be the distribution specified by :

$$
\forall \phi \in \mathcal{S}(\mathbb{R}),<(\ln t)_{+}^{k} t_{+}^{-s-\alpha} ; \phi>=<D^{s}\left(F_{k, s, \alpha}\right) ; \phi>=<F_{k, s, \alpha} ; \phi^{(s)}>\cdot(-1)^{s}
$$

From (14) we have

$$
f_{n+1}(t)=f_{n}(t)-\frac{a_{n}(\ln t)^{k}}{t^{n+\alpha}}
$$

Integrating $n$ times (with value 0 at $+\infty$ ) :

$$
f_{n+1, n}(t)=f_{n, n}(t)-a_{n} F_{k, n, \alpha}(t)
$$

Multiplying by $(-1)^{n} \phi^{(n)}$ and integrating :

$$
\begin{aligned}
<f_{n} ; \phi>= & (-1)^{n} \int_{0}^{+\infty} f_{n+1, n}(t) \phi^{(n)}(t) d t+a_{n}(-1)^{n} \int_{0}^{+\infty} F_{k, n, \alpha}(t) \phi^{(n)}(t) d t \\
= & (-1)^{n} \int_{0}^{+\infty} f_{n+1, n}(t) \phi^{(n)}(t) d t+a_{n}<(\ln t)_{+}^{k} t_{+}^{-n-\alpha} ; \phi> \\
= & (-1)^{n}\left(\left[f_{n+1, n+1}(t) \phi^{(n)}(t)\right]_{0}^{\infty}-\int_{0}^{+\infty} f_{n+1, n+1}(t) \phi^{(n+1)}(t) d t\right) \\
& +a_{n}<(\ln t)_{+}^{k} t_{+}^{-n-\alpha} ; \phi> \\
= & -f_{n+1, n+1}(0)<\delta^{(n)} ; \phi>+<f_{n+1}, \phi>+a_{n}<(\ln t)_{+}^{k} t_{+}^{-n-\alpha} ; \phi>
\end{aligned}
$$


and setting $c_{n+1}=f_{n+1, n+1}(0)$

$$
<f_{n+1}, \phi>+a_{n}<(\ln t)_{+}^{k} t_{+}^{-n-\alpha} ; \phi>=<f_{n}, \phi>+c_{n+1}<\delta^{(n)} ; \phi>
$$

then

$$
\begin{gathered}
<f_{n+1}, \phi>+\sum_{s=0}^{n} a_{s}<(\ln t)_{+}^{k} t_{+}^{-s-\alpha} ; \phi> \\
=<f_{n}, \phi>+\sum_{s=0}^{n-1} a_{s}<(\ln t)_{+}^{k} t_{+}^{-s-\alpha} ; \phi>+c_{n+1}<\delta^{(n)} ; \phi> \\
=\ldots=<f ; \phi>+\sum_{s=1}^{n+1} c_{s}<\delta^{(s-1)} ; \phi>
\end{gathered}
$$

2) Case $\alpha=1$.

We extend Lemma 6 to functions $f$ satisfying $f(t) \sim \sum_{s=0}^{\infty} \frac{a_{s}(\ln t)^{k}}{t^{s+1}}$, as $t \rightarrow+\infty$ We define $F_{k, s, 1}$ as in (16). $(\ln t)_{+}^{k} t_{+}^{-s-1}$ will be the distribution on $\mathbb{R}$ specified by:

$$
\begin{aligned}
\forall \phi \in \mathcal{S}(\mathbb{R}),<(\ln t)_{+}^{k} t_{+}^{-s-1} ; \phi> & =<D^{s+1}\left(F_{k, s, 1}\right) ; \phi>=<F_{k, s, 1} ; \phi^{(s+1)}>\cdot(-1)^{s+1} \\
f_{n+1}(t) & =f_{n}(t)-\frac{a_{n}(\ln t)^{k}}{t^{n+1}}
\end{aligned}
$$

Integrating $n$ times (with value 0 at $+\infty$ ) :

$$
f_{n+1, n}(t)=f_{n, n}(t)-a_{n} F_{k, n, 1}^{\prime}(t)
$$

From (18), $f_{n+1, n+1}(t)+a_{n} F_{k, n, 1}(t)$ is a primitive of $f_{n, n}(t)$.

$\int_{0}^{t} f_{n, n}(\tau) d \tau$ is another one then there exists a constant $c_{n+1}$ such that

$$
c_{n+1}+\int_{0}^{t} f_{n, n}(\tau) d \tau=f_{n+1, n+1}(t)+a_{n} F_{k, n, 1}(t)
$$

and $c_{n+1}=\lim _{t \rightarrow 0}\left(f_{n+1, n+1}(t)+a_{n} F_{k, n, 1}(t)\right)$

From (18) we have for $\phi \in \mathcal{S}(\mathbb{R})$ :

$$
\begin{aligned}
& (-1)^{n} \int_{0}^{+\infty} f_{n, n}(t) \phi^{(n)}(t) d t=(-1)^{n} \int_{0}^{+\infty}\left(f_{n+1, n}(t)+a_{n} F_{k, n, 1}^{\prime}(t)\right) \phi^{(n)}(t) d t \\
= & (-1)^{n}\left[\left(f_{n+1, n+1}(t)+a_{n} F_{k, n, 1}(t)\right) \phi^{(n)}(t)\right]_{0}^{\infty} \\
& -(-1)^{n} \int_{0}^{+\infty}\left(f_{n+1, n+1}(t)+a_{n} F_{k, n, 1}(t)\right) \phi^{(n+1)}(t) d t \quad \text { (by parts) } \\
= & (-1)^{n+1} c_{n+1} \phi^{(n)}(0)+<f_{n+1} ; \phi>+(-1)^{n+1} a_{n} \int_{0}^{\infty} F_{k, n, 1}(t) \phi^{(n+1)}(t) d t
\end{aligned}
$$

i.e.:

$$
<f_{n+1} ; \phi>+a_{n}<(\ln (t))_{+}^{k} t_{+}^{-n-1} ; \phi>=<f_{n} ; \phi>+c_{n+1}<\delta^{(n)} ; \phi>
$$


then

$$
\begin{gathered}
<f_{n+1} ; \phi>+\sum_{s=0}^{n} a_{s}<(\ln (t))_{+}^{k} t_{+}^{-s-1} ; \phi> \\
=<f_{n} ; \phi>+\sum_{s=0}^{n-1} a_{s}<(\ln (t))_{+}^{k} t_{+}^{-s-1} ; \phi>+c_{n+1}<\delta^{(n)} ; \phi> \\
=\ldots=<f ; \phi>+\sum_{s=1}^{n+1} c_{s}<\delta^{(s-1)} ; \phi>
\end{gathered}
$$

this ends the proof of Lemma 7 .

4.2. Second step of the proof of Proposition 6. We show now that $M_{B}$ can be computed, for $w$ stated, with Lemma 7 . We will integrate then in $w$ which will give a decomposition (22) of $M_{B}$ in 3 terms evaluated further. Let us set :

$$
\begin{aligned}
\phi_{\lambda}(w ; t) & =\exp \left(\frac{1}{\lambda} \sum_{\mu \in \Delta \backslash\{\nu\}} a_{\mu} w^{\beta_{1}(\mu)} \cdot t^{\beta_{2}(\mu)}\right) w^{b_{1}-1} \cdot t^{c^{+}}(\ln w)^{B_{1}} \\
f_{\lambda}(w ; t) & =\frac{(\ln t)^{k}}{t^{\alpha+c^{-}}} \cdot \exp \left(\frac{1}{\lambda} a_{\nu} w^{\beta_{1}(\nu)} t^{\beta_{2}(\nu)}\right) .
\end{aligned}
$$

Then $M_{B}=\int_{\mathbb{R}_{+}}\left[\int_{\mathbb{R}_{+}} \phi_{\lambda}(w ; t) f_{\lambda}(w ; t) d t\right] d w$

For $\lambda$ and $w$ stated $(w \neq 0), \phi_{\lambda}(w ; t)$ and $f_{\lambda}(w ; t)$ satisfy the hypotheses of Lemma 7 , which will allow us to compute $\int_{\mathbb{R}_{+}} \phi_{\lambda}(w ; t) f_{\lambda}(w ; t) d t$

The map $t \mapsto \phi_{\lambda}(w ; t)$ is indeed in $\mathcal{S}(\mathbb{R})$ because $\beta_{2}(\mu) \geq 0$ for $\mu \in \Sigma(P) \backslash\{\nu\}=$ $\left\{\mu \in \Delta \backslash\{\nu\}, a_{\mu} \neq 0\right\}$. Recall that $\beta_{2}(\nu)<0$.

$$
f_{\lambda}(w ; t) \sim \sum_{s=0}^{\infty} \frac{a_{s}(\lambda, w)}{t^{\alpha+s}} .(\ln t)^{k} \quad \text { as } t \rightarrow+\infty
$$

where $a_{s}(\lambda, w)$ is a polynomial in $w$ :

$$
\begin{aligned}
& a_{s}(\lambda, w)=\frac{1}{\bar{r} !} \cdot\left(\frac{a_{\nu} w^{\beta_{1}(\nu)}}{\lambda}\right)^{\bar{r}} \text { for } s=c^{-}+\bar{r} \cdot\left(-\beta_{2}(\nu)\right) \text { with } \bar{r} \in \mathbb{N} \\
& a_{s}(\lambda, w)=0 \quad \text { else. }
\end{aligned}
$$

From Lemma 7, for any $n \geq 1$ :

$$
\begin{gathered}
\int_{0}^{\infty} \phi_{\lambda}(w ; t) f_{\lambda}(w ; t) d t=\sum_{s=0}^{n-1} a_{s}(\lambda ; w)<(\ln t)_{+}^{k} t_{+}^{-s-\alpha} ; \phi_{\lambda}> \\
-\sum_{s=1}^{n} c_{s}(\lambda ; w)<\delta^{(s-1)} ; \phi_{\lambda}>+<\left(f_{\lambda}\right)_{n} ; \phi_{\lambda}>
\end{gathered}
$$

where $<\delta^{(s-1)} ; \phi_{\lambda}>=(-1)^{s-1} \frac{\partial^{s-1} \phi_{\lambda}}{\partial t^{s-1}}(w, 0)$

- If $0<\alpha<1$, the $c_{s}(\lambda ; w)$ are defined by $c_{s}(\lambda ; w)=\left(f_{\lambda}\right)_{s, s}(w ; 0)$

- If $\alpha=1$, according to Lemma 7

$$
c_{s}(\lambda ; w)=\lim _{t \rightarrow 0}\left(\left(f_{\lambda}\right)_{s, s}(w ; t)+a_{s-1}(\lambda ; w) F_{k ; s-1 ; 1}(t)\right)
$$


To complete the proof of Proposition 6, we will study successively the asymptotic expansions of the three terms in

$$
M_{B}=I_{1}+I_{2}+I_{3}
$$

where

$$
\begin{aligned}
& I_{1}=\int_{\mathbb{R}_{+}}\left[\sum_{s=0}^{n-1} a_{s}(\lambda, w)<(\ln t)_{+}^{k} t_{+}^{-s-\alpha} ; \phi_{\lambda}>\right] d w \\
& I_{2}=-\int_{\mathbb{R}_{+}}\left[\sum_{s=1}^{n} c_{s}(\lambda, w)<\delta^{(s-1)} ; \phi_{\lambda}>\right] d w \\
& I_{3}=\int_{\mathbb{R}_{+}}<\left(f_{\lambda}\right)_{n} ; \phi_{\lambda}>d w
\end{aligned}
$$

4.3. End of the proof of Proposition 6. We will now study each of these three integrals, to state there exist constants $c_{i}^{\prime}$ and $c_{j, l}^{\prime}$ such that

$$
\begin{aligned}
& I_{1}=\sum_{i} c_{i}^{\prime} \lambda^{-i} \cdot L\left(\theta_{i}, \gamma_{i}, P_{\Delta *}\right) \\
& I_{2}=\sum_{j, l} c_{j, l}^{\prime} \lambda^{j} .(\ln \lambda)^{l} \\
& I_{3}=o\left(\lambda^{M}\right) \quad \text { as } \lambda \rightarrow 0^{+} \text {for } n \text { sufficiently large. }
\end{aligned}
$$

where the sums have a finite number of terms, $i \in \mathbb{N}, l \in \mathbb{N}$. We will see in (39) that to have $I_{3}=o\left(\lambda^{M}\right)$, it is sufficient to take $n=c^{-}+\left(-\beta_{2}(\nu)\right) \bar{R}$ where $\bar{R}$ is the integer part of $\frac{M-A_{\Delta^{*}}\left(b^{\prime}\right)}{A_{\Delta^{*}}(\nu)-1}+1$

4.3.1. Study of $I_{1}$. The case $\left.\alpha \in\right] 0 ; 1[$ is first considered. Fix $w \neq 0$.

$$
<(\ln t)_{+}^{k} t_{+}^{-s-\alpha} ; \phi_{\lambda}>=(-1)^{s} \int_{0}^{\infty} F_{k, s, \alpha}(t) \cdot \frac{\partial^{s} \phi_{\lambda}}{\partial t^{s}}(w ; t) d t
$$

where $F_{k, s, \alpha}(t)$ is defined in (15). According to (15), we have

$$
<(\ln t)_{+}^{k} t_{+}^{-s-\alpha} ; \phi_{\lambda}>=\sum_{r=0}^{k} K(k, s, \alpha, r) . I_{s, r}
$$

where, for $r \in\{0 ; . . ; k\}$

$$
I_{s, r}=\int_{0}^{\infty}(\ln t)^{r} \cdot \frac{\partial^{s} \phi_{\lambda}}{\partial t^{s}}(w ; t) \frac{d t}{t^{\alpha}}
$$

Computation of $\frac{\partial^{s} \phi_{\lambda}}{\partial t^{s}}(w ; t)$

From (19) :

$$
\phi_{\lambda}(w ; t)=\exp \left(\frac{1}{\lambda} \sum_{\mu \in \Delta \backslash\{\nu\}} a_{\mu} w^{\beta_{1}(\mu)} t^{\beta_{2}(\mu)}\right) \cdot w^{b_{1}-1} t^{c^{+}} .(\ln w)^{B_{1}}
$$


Derivating $s$ times with respect to $t$ :

$$
\begin{aligned}
\frac{\partial^{s} \phi_{\lambda}}{\partial t^{s}}(w ; t)= & w^{b_{1}-1}(\ln w)^{B_{1}} \sum_{j=0}^{s}\left(\begin{array}{c}
s \\
j
\end{array}\right) \frac{\partial^{s-j}\left(t^{c^{+}}\right)}{\partial t^{s-j}} \\
& \cdot \frac{\partial^{j}}{\partial t^{j}}\left(\exp \left(\frac{1}{\lambda} \sum_{\mu \in \Delta \backslash\{\nu\}} a_{\mu} w^{\beta_{1}(\mu)} t^{\beta_{2}(\mu)}\right)\right)
\end{aligned}
$$

where

$$
\begin{aligned}
\frac{\partial^{s-j}\left(t^{c^{+}}\right)}{\partial t^{s-j}} & =\frac{\left(c^{+}\right) !}{\left(c^{+}-s+j\right) !} t^{\left(c^{+}-s+j\right)} \text { for } \quad c^{+} \geq s-j \\
& =0 \text { for } \quad c^{+}<s-j
\end{aligned}
$$

It remains to evaluate $\frac{\partial^{j}}{\partial t^{j}}\left(\exp \left(\frac{1}{\lambda} \sum_{\mu \in \Delta \backslash\{\nu\}} a_{\mu} w^{\beta_{1}(\mu)} t^{\beta_{2}(\mu)}\right)\right)$ for $1 \leq j \leq s$ Let us recall that if $\mu \in \Sigma(P) \backslash\{\nu\}$ we have $\beta_{2}(\mu) \in \mathbb{N}$. Then:

$$
\begin{gathered}
\frac{\partial}{\partial t}\left(\exp \left(\frac{1}{\lambda} \sum_{\mu \in \Delta \backslash\{\nu\}} a_{\mu} w^{\beta_{1}(\mu)} t^{\beta_{2}(\mu)}\right)\right) \\
=\left(\frac{1}{\lambda} \sum_{\mu \in \Delta \backslash\{\nu\}} \beta_{2}(\mu) \cdot a_{\mu} w^{\beta_{1}(\mu)} t^{\beta_{2}(\mu)-1}\right) \cdot \exp \left(\frac{1}{\lambda} \sum_{\mu \in \Delta \backslash\{\nu\}} a_{\mu} w^{\beta_{1}(\mu)} t^{\beta_{2}(\mu)}\right)
\end{gathered}
$$

Likewise:

$$
\begin{aligned}
& \frac{\partial^{2}}{\partial t^{2}}\left(\exp \left(\frac{1}{\lambda} \sum_{\mu \in \Delta \backslash\{\nu\}} a_{\mu} w^{\beta_{1}(\mu)} t^{\beta_{2}(\mu)}\right)\right) \\
& =\exp \left(\frac{1}{\lambda} \sum_{\mu \in \Delta \backslash\{\nu\}} a_{\mu} w^{\beta_{1}(\mu)} t^{\beta_{2}(\mu)}\right)\left[\frac{1}{\lambda} \sum_{\mu \in \Delta \backslash\{\nu\}} \beta_{2}(\mu) \cdot\left(\beta_{2}(\mu)-1\right) \cdot a_{\mu} w^{\beta_{1}(\mu)} t^{\beta_{2}(\mu)-2}\right. \\
& +\frac{1}{\lambda^{2}} \sum_{\bar{\mu}(1), \bar{\mu}(2) \in \Delta \backslash\{\nu\}} \beta_{2}(\bar{\mu}(1)) \cdot \beta_{2}(\bar{\mu}(2)) \cdot a_{\bar{\mu}(1)} \cdot a_{\bar{\mu}(2)} \cdot w^{\beta_{1}(\bar{\mu}(1))+\beta_{1}(\bar{\mu}(2))} \\
& \left.. t^{\left(\beta_{2}(\bar{\mu}(1))+\beta_{2}(\bar{\mu}(2))-2\right)}\right]
\end{aligned}
$$

and finally by induction on $j$ :

$$
\begin{gathered}
\frac{\partial^{j}}{\partial t^{j}}\left(\exp \left(\frac{1}{\lambda} \sum_{\mu \in \Delta \backslash\{\nu\}} a_{\mu} w^{\beta_{1}(\mu)} t^{\beta_{2}(\mu)}\right)\right) \\
=\sum_{\rho=1}^{j} \sum_{\bar{\mu} \in S_{\rho}, \beta_{2}(\bar{\mu}) \geq j} \text { const. } \frac{1}{\lambda^{\rho}} \cdot w^{\beta_{1}(\bar{\mu})} \cdot t^{\beta_{2}(\bar{\mu})-j} \cdot \exp \left(\frac{1}{\lambda} \sum_{\mu \in \Delta \backslash\{\nu\}} a_{\mu} w^{\beta_{1}(\mu)} t^{\beta_{2}(\mu)}\right)
\end{gathered}
$$

where $\mathcal{S}_{\rho}=\left\{\bar{\mu} ; \bar{\mu}=\sum_{i=1}^{\rho} \bar{\mu}(i) \quad\right.$ where the $\left.\bar{\mu}(i) \in \Sigma(P) \backslash\{\nu\}, \beta_{2}(\bar{\mu}(i)) \geq 1\right\}$. Moreover we set $\mathcal{S}_{\rho}=\{0\}$ if $\rho=0$. 
Equations (24) and (25) finally give:

$$
\begin{aligned}
\frac{\partial^{s} \phi_{\lambda}}{\partial t^{s}}(w ; t)= & \exp \left(\frac{1}{\lambda} \sum_{\mu \in \Delta \backslash\{\nu\}} a_{\mu} w^{\beta_{1}(\mu)} t^{\beta_{2}(\mu)}\right) \cdot w^{b_{1}-1} \cdot(\ln w)^{B_{1}} \\
& \cdot \sum_{\rho=0}^{s} \sum_{\bar{\mu} \in \mathcal{S}_{\rho}} h_{s}(\rho, \bar{\mu}) \cdot \frac{1}{\lambda^{\rho}} \cdot w^{\beta_{1}(\bar{\mu})} \cdot t^{\beta_{2}(\bar{\mu})+c^{+}-s}
\end{aligned}
$$

where

$$
h_{s}(\rho, \bar{\mu})=0 \text { if } \beta_{2}(\bar{\mu})<s-c^{+}
$$

$\rho=0$ is possible if $c^{+}-s \geq 0$.

$I_{s, r}$ defined in (23) becomes:

$$
\begin{aligned}
I_{s, r}= & \sum_{\rho=0}^{s} \sum_{\bar{\mu} \in \mathcal{S}_{\rho}} h_{s}(\rho, \bar{\mu}) \cdot \frac{1}{\lambda^{\rho}}(\ln w)^{B_{1}} w^{\beta_{1}(\bar{\mu})+b_{1}-1} \\
& \cdot \int_{0}^{\infty}(\ln t)^{r} \cdot \exp \left(\frac{1}{\lambda} \sum_{\mu \in \Delta \backslash\{\nu\}} a_{\mu} w^{\beta_{1}(\mu)} t^{\beta_{2}(\mu)}\right) \cdot t^{\beta_{2}(\bar{\mu})+c^{+}-s} \cdot \frac{d t}{t^{\alpha}}
\end{aligned}
$$

where each integral in (28) is convergent because according to (27),

$$
\beta_{2}(\bar{\mu})+c^{+}-s-\alpha \geq s-c^{+}+c^{+}-s-\alpha \geq-\alpha>-1
$$

To compute $I_{1}$ one must integrate with respect to $w$ (we will justify later the integrability with respect to $w$ )

$$
I_{1}=\sum_{s=0}^{n-1} \int_{\mathbb{R}_{+}} a_{s}(\lambda, w)<(\ln t)_{+}^{k} t_{+}^{-s-\alpha} ; \phi_{\lambda}>d w
$$

where

$$
\begin{aligned}
& \int_{\mathbb{R}_{+}} a_{s}(\lambda, w)<(\ln t)_{+}^{k} t_{+}^{-s-\alpha} ; \phi_{\lambda}>d w \\
= & \sum_{r=0}^{k} K(k, s, \alpha, r) \int_{\mathbb{R}_{+}} I_{s, r}(w) \cdot a_{s}(\lambda, w) \cdot d w
\end{aligned}
$$

(because $I_{s, r}=I_{s, r}(w)$ in $(23)$ )

By (28) and (29), there exist constants $\delta(s, r, \rho, \bar{\mu}, k, \alpha)$ such that:

$$
\begin{aligned}
I_{1}= & \sum_{s=0}^{n-1} \int_{\mathbb{R}_{+}} a_{s}(\lambda, w)<(\ln t)_{+}^{k} t_{+}^{-s-\alpha} ; \phi_{\lambda}>d w \\
= & \sum_{s, r, \rho, \bar{\mu}} \delta(s, r, \rho, \bar{\mu}, k, \alpha) \cdot \frac{1}{\lambda^{\rho}} \int_{\mathbb{R}_{+}^{2}} a_{s}(\lambda, w) \cdot w^{\beta_{1}(\bar{\mu})+b_{1}-1}(\ln w)^{B_{1}} \\
& (\ln t)^{r} \cdot t^{\beta_{2}(\bar{\mu})+c^{+}-s} \cdot \exp \left(\frac{1}{\lambda} \sum_{\mu \in \Delta \backslash\{\nu\}} a_{\mu} \cdot w^{\beta_{1}(\mu)} t^{\beta_{2}(\mu)}\right) \cdot \frac{d t}{t^{\alpha}} d w
\end{aligned}
$$


where: $\quad 0 \leq s \leq n-1, \quad 0 \leq r \leq k, \quad 0 \leq \rho \leq s$ $\bar{\mu}=\bar{\mu}(1)+. .+\bar{\mu}(\rho)$, if $\rho \geq 1$, the $\bar{\mu}(i) \in \Sigma(P) \backslash\{\nu\}$, and $\bar{\mu}=0$ if $\rho=0$.

Let us recall that $w^{D}=x^{\mu(1)}, t^{D}=x^{\mu(2)}$, and $w^{\beta_{1}(\mu)} t^{\beta_{2}(\mu)}=x^{\mu}$. Coming back to $x$ :

$$
\begin{aligned}
I_{1}= & \sum_{s, r, \rho, \bar{\mu}} \delta(s, r, \rho, \bar{\mu}, k, \alpha) \cdot \frac{1}{\lambda^{\rho}} \int_{\mathbb{R}_{+}^{2}} a_{s}\left(\lambda, x^{\frac{\mu(1)}{D}}\right) \\
& . x^{\bar{\mu}} \cdot\left[\ln \left(x^{\frac{\mu(1)}{D}}\right)\right]^{B_{1}} \cdot\left[\ln \left(x^{\frac{\mu(2)}{D}}\right)\right]^{r} x^{\frac{\mu(2)}{D}\left(c^{+}+1-s-\alpha\right)} \\
& . x^{\frac{1}{D} \cdot b_{1} \mu(1)} \cdot \exp \left(\frac{P^{*}(x)}{\lambda}\right) \cdot \frac{d x}{D \prod_{i} x_{i}}
\end{aligned}
$$

where $P^{*}(x)=\sum_{\mu \in \Delta \backslash\{\nu\}} a_{\mu} x^{\mu}=\sum_{\mu \in \Delta^{*}} a_{\mu} x^{\mu}, \Delta^{*}$ is the Newton diagram of $P^{*}$.

Recall that $a_{s}$ is the polynomial defined from (20):

$$
\begin{aligned}
& a_{s}(\lambda, w)=\frac{1}{\bar{r} !} \cdot\left(\frac{a_{\nu} w^{\beta_{1}(\nu)}}{\lambda}\right)^{\bar{r}} \quad \text { for } \bar{r}=\frac{s-c^{-}}{\left(-\beta_{2}(\nu)\right)} \in \mathbb{N} \\
& a_{s}(\lambda, w)=0 \quad \text { else. }
\end{aligned}
$$

thus

$$
a_{s}(\lambda, w)=a_{s}\left(\lambda, x^{\frac{\mu(1)}{D}}\right)=\frac{1}{\lambda^{\bar{r}}} \frac{a_{\nu}^{\bar{r}}}{\bar{r} !} x^{\overline{\bar{T}}} \cdot \beta_{1}(\nu) \mu(1)
$$

Expanding in (31), there are constants $b_{\Sigma}^{\prime}$ such that

$$
\left[\ln \left(x^{\frac{\mu(1)}{D}}\right)\right]^{B_{1}} \cdot\left[\ln \left(x^{\frac{\mu(2)}{D}}\right)\right]^{r}=\sum_{|\Sigma|=B_{1}+r} b_{\Sigma}^{\prime}(\ln x)^{\Sigma}
$$

where $\Sigma \in \mathbb{N}^{2},|\Sigma|=\Sigma_{1}+\Sigma_{2},(\ln x)^{\Sigma}=\prod_{i}\left(\ln x_{i}\right)^{\Sigma_{i}}$

It is clear that $a_{s}=0$ if $s<c^{-}$.

By (31), (32) and as $c^{+}+1-s-\alpha=c^{+}-s+b_{2}-c=b_{2}+c^{-}-s$ (from (11)), there exist constants $\delta^{\prime}(\bar{r}, r, \rho, \bar{\mu}, k, \alpha, \Sigma)$ such that :

$$
\begin{aligned}
I_{1}= & \sum_{\bar{r}, j, . ., \Sigma} \delta^{\prime}(\bar{r}, r, \rho, \bar{\mu}, k, \alpha, \Sigma) \cdot \lambda^{-\bar{r}-\rho} \int_{\mathbb{R}_{+}^{2}} x^{\bar{\mu}} \cdot x^{\frac{\overline{\bar{D}}}{D} \cdot \beta_{1}(\nu) \mu(1)} \\
& x^{\frac{1}{D} \cdot b_{1} \mu(1)} x^{\frac{\mu(2)}{D}\left(b_{2}+c^{-}-s\right)} \cdot(\ln x)^{\Sigma} \exp \left(\frac{P^{*}(x)}{\lambda}\right) \cdot \frac{d x}{\prod_{i} x_{i}}
\end{aligned}
$$

where $\bar{r} \in \mathbb{N}, \bar{r}=\frac{s-c^{-}}{-\beta_{2}(\nu)}$.

$$
\begin{aligned}
I_{1}= & \sum_{\text {finite }} \delta^{\prime}(\bar{r}, r, \rho, \bar{\mu}, k, \alpha, \Sigma) \cdot \lambda^{-\bar{r}-\rho} \int_{\mathbb{R}_{+}^{2}} x^{\bar{\mu}} \cdot x^{\frac{\mu(1)}{D}\left(b_{1}+\beta_{1}(\nu) \cdot \bar{r}\right)} x^{\frac{\mu(2)}{D}\left(c^{-}+b_{2}-s\right)} \\
& .(\ln x)^{\Sigma} \exp \left(\frac{P^{*}(x)}{\lambda}\right) \cdot \frac{d x}{\prod_{i} x_{i}}
\end{aligned}
$$

And since $\frac{1}{D}\left(b_{1} \mu(1)+b_{2} \mu(2)\right)=b^{\prime}$ (see Lemma 5 with $\left.b_{i}=\beta_{i}\left(b^{\prime}\right)\right)$

$$
\begin{aligned}
I_{1}= & \sum_{\text {finite }} \delta^{\prime}(\bar{r}, r, \rho, \bar{\mu}, k, \alpha, \Sigma) \cdot \lambda^{-\bar{r}-\rho} \int_{\mathbb{R}_{+}^{2}} x^{\bar{\mu}} \cdot x^{\frac{\mu(1)}{D}\left(\beta_{1}(\nu) \cdot \bar{r}\right)} \cdot x^{b^{\prime}} x^{-\frac{\mu(2)}{D}\left(s-c^{-}\right)} \\
& .(\ln x)^{\Sigma} \exp \left(\frac{P^{*}(x)}{\lambda}\right) \cdot \frac{d x}{\prod_{i} x_{i}}
\end{aligned}
$$


where

$$
\begin{aligned}
& \frac{\mu(1)}{D} \bar{r} \beta_{1}(\nu)-\frac{\mu(2)}{D}\left(s-c^{-}\right) \\
= & \frac{\mu(1)}{D} \bar{r} \beta_{1}(\nu)+\frac{1}{D} \mu(2) \bar{r} \beta_{2}(\nu)=\frac{\bar{r}}{D} \sum_{i=1}^{2} \beta_{i}(\nu) \mu(i)=\nu . \bar{r}
\end{aligned}
$$

then

$$
\begin{aligned}
I_{1} & =\sum_{\text {finite }} \delta^{\prime}(\bar{r}, r, \rho, \bar{\mu}, k, \alpha, \Sigma) \cdot \lambda^{-\bar{r}-\rho} \int_{\mathbb{R}_{+}^{2}} x^{\bar{\mu}} \cdot x^{\bar{r} \nu} \cdot x^{b^{\prime}-1} \cdot(\ln x)^{\Sigma} \exp \left(\frac{P^{*}(x)}{\lambda}\right) \cdot d x \\
& =\sum_{\text {finite }} \delta^{\prime}(\bar{r}, r, \rho, \bar{\mu}, k, \alpha, \Sigma) \cdot \lambda^{-\bar{r}-\rho} L\left(\bar{\mu}+b^{\prime}+\bar{r} \nu, \Sigma, P^{*}\right)
\end{aligned}
$$

We will show that each term here has the same form than our integral $L\left(b^{\prime}, b^{\prime \prime}, P\right)$ but with a Newton Diagram including one vertex less. We had:

$$
L\left(b^{\prime}, b^{\prime \prime}, P\right)=\int_{\mathbb{R}_{+}^{2}} \exp \left(\frac{P(x)}{\lambda}\right) \cdot x^{b^{\prime}-1}(\ln x)^{b^{\prime \prime}} d x
$$

where $\left(b^{\prime}, b^{\prime \prime}, P\right)$ satisfies Hypothesis HP.

Here $L\left(\bar{\mu}+b^{\prime}+\bar{r} \nu, \Sigma, P^{*}\right)$ will be of the same form than $L\left(b^{\prime}, b^{\prime \prime}, P\right)$ with $P^{*}(x)$ instead of $P_{\Delta}(x)$. We have $\Sigma \in \mathbb{N}^{2}$. We want that $\left(\bar{\mu}+b^{\prime}+\bar{r} \nu, \Sigma, P_{\Delta \backslash\{\nu\}}\right)$ satisfies Hypothesis HP.

The only thing we must check is that

$$
\tilde{\mu}=\bar{\mu}+b^{\prime}+\bar{r} . \nu \text { is in the interior of } \mathcal{C}\left(\Delta^{*}\right)
$$

where $\Delta^{*}$ is the Newton Diagram of $P^{*}(x)=P_{\Delta \backslash\{\nu\}}(x)$.

We know that $b^{\prime} \in \operatorname{int} \mathcal{C}(\Delta) . \bar{\mu}=\sum_{i=1}^{p} \bar{\mu}(i)$, where the $\bar{\mu}(i)$ and $\nu$ are in $\mathcal{C}(\Delta)$ then $\tilde{\mu} \in \operatorname{int} \mathcal{C}(\Delta)$. It remains therefore to prove that $\tilde{\mu}$ is on the right side of the line $(0 ; \mu(1))$ separating $\nu$ from $\Delta^{*}$, i.e. is it true that $\beta_{2}(\tilde{\mu})>0$ ?

$$
\begin{aligned}
\beta_{2}(\tilde{\mu}) & =\operatorname{det}(\mu(1), \tilde{\mu})=\beta_{2}\left(b^{\prime}\right)+\beta_{2}(\bar{\mu})+\bar{r} \cdot \beta_{2}(\nu) \\
& \geq \beta_{2}\left(b^{\prime}\right)+\left(s-c^{+}\right)+\bar{r} \cdot \beta_{2}(\nu)=b_{2}+s-c^{+}-\left(s-c^{-}\right) \quad(\text { from }(27)) \\
& =b_{2}-c=1-\alpha>0 \quad(\text { from }(11) \text { and because } 0<\alpha<1)
\end{aligned}
$$

and we just obtain what we wanted.

We have then in (33) integrals $L\left(\tilde{\mu}, \Sigma, P^{*}\right)$ of the same form that the initial integral $L\left(b^{\prime}, b^{\prime}, P\right)$ but with a vertex less and a facet less (this proves also that they are quite convergent). We have thus showed that in the expression (22) i.e. $M_{B}=I_{1}+I_{2}+I_{3}$, the first term $I_{1}$ is a linear combination of integrals of the form of $L\left(b^{\prime}, b^{\prime \prime}, P\right)$, but with a vertex less than $L\left(b^{\prime}, b^{\prime \prime}, P\right)$. 
Sketch of the case $\alpha=1$.

$$
<(\ln t)_{+}^{k} t_{+}^{-s-1} ; \phi_{\lambda}>=(-1)^{s+1} \int_{0}^{\infty} F_{k, s, 1}(t) \cdot \frac{\partial^{s+1} \phi_{\lambda}}{\partial t^{s+1}}(w ; t) d t
$$

$I_{s, r}$ becomes $I_{s, r}=\int_{0}^{\infty}(\ln t)^{r} \cdot \frac{\partial^{s+1} \phi_{\lambda}}{\partial t^{s+1}}(w ; t) d t, r \in\{1 ; . . ; k+1\}$

In (26) and after, one must then evaluate $\frac{\partial^{s+1} \phi_{\lambda}}{\partial t^{s+1}}(w ; t) d t$ instead of $\frac{\partial^{s} \phi_{\lambda}}{\partial t^{s}}(w ; t) d t$

We obtain a finite sum of integrals $L\left(\tilde{\mu}, \Sigma, P_{\Delta \backslash\{\nu\}}\right)$ with:

$$
\begin{aligned}
\beta_{2}(\widetilde{\mu}) & =\beta_{2}(\bar{\mu})+\beta_{2}\left(b^{\prime}\right)+\bar{r} \beta_{2}(\nu) \geq(s+1)-c^{+}+b_{2}-\left(s-c^{-}\right) \\
& =s+1-c^{+}+c-s+c^{-}=1>0
\end{aligned}
$$

The remainder of the proof is similar to that of the case $\alpha<1$.

\subsubsection{Study of $I_{2}$.}

$$
I_{2}=-\int_{\mathbb{R}_{+}}\left[\sum_{s=1}^{n} c_{s}(\lambda, w)<\delta^{(s-1)} ; \phi_{\lambda}>\right] d w
$$

$I_{2}$ is convergent because in (22), $M_{B}, I_{1}$ and $I_{3}$ are convergent. This is true for all $n \in$ $\mathbb{N}^{*}$, then by subtracting the values in $(n+1)$ and in $n$, one finds that $\int_{\mathbb{R}_{+}} c_{s}(\lambda, w)<$ $\delta^{(s-1)} ; \phi_{\lambda}>d w$ is convergent.

$$
<\delta^{(s-1)} ; \phi_{\lambda}>=(-1)^{s-1} \frac{\partial^{s-1} \phi_{\lambda}}{\partial t^{s-1}}(w ; 0)
$$

where $\frac{\partial^{s-1} \phi_{\lambda}}{\partial t^{s-1}}$ is evaluated with $(26)$.

$$
\begin{aligned}
\frac{\partial^{s-1} \phi_{\lambda}}{\partial t^{s-1}}(w ; t)= & \exp \left(\frac{1}{\lambda} \sum_{\mu \in \Delta \backslash\{\nu\}} a_{\mu} w^{\beta_{1}(\mu)} t^{\beta_{2}(\mu)}\right) \\
& \sum_{\rho=0}^{s-1} \sum_{\bar{\mu} \in \mathcal{S}_{\rho}} h_{s-1}(\rho, \bar{\mu}) \cdot(\ln w)^{B_{1}} \cdot \lambda^{-\rho} w^{\beta_{1}(\bar{\mu})} w^{b_{1}-1} t^{c^{+}-s+1+\beta_{2}(\bar{\mu})}
\end{aligned}
$$

For $t=0$, all the terms vanish except those for which the power of $t$ is null, i.e. when $\beta_{2}(\bar{\mu})+c^{+}-s+1=0$. Since $\beta_{2}(\bar{\mu}) \geq \rho$, one must have $\rho \leq s-1-c^{+}$.

In the exponential, $\beta_{2}(\mu)>0$ except for $\mu=\mu(1)$.

$$
\begin{aligned}
\frac{\partial^{s-1} \phi_{\lambda}}{\partial t^{s-1}}(w ; 0)= & \exp \left(\frac{1}{\lambda} a_{\mu(1)} \cdot w^{\beta_{1}(\mu(1))}\right) \\
& \cdot \sum_{\rho=0}^{s-1-c^{+}} \sum_{\bar{\mu} \in \mathcal{S}_{\rho}, \beta_{2}(\bar{\mu})=s-c^{+}-1} h_{s-1}(\rho, \bar{\mu}) .(\ln w)^{B_{1}} \cdot \lambda^{-\rho} w^{\beta_{1}(\bar{\mu})+b_{1}-1}
\end{aligned}
$$

(it is null if $s-1-c^{+}<0$ because $0 \leq \rho \leq s-1-c^{+}$)

$$
\begin{aligned}
& \int_{\mathbb{R}_{+}} c_{s}(\lambda, w)<\delta^{(s-1)} ; \phi_{\lambda}>d w \\
= & \int_{\mathbb{R}_{+}}(-1)^{s-1} \sum_{\rho=0}^{s-1-c^{+}} \sum_{\bar{\mu} \in \mathcal{S}_{\rho}, \beta_{2}(\bar{\mu})=s-c^{+}-1} h_{s-1}(\rho, \bar{\mu}) \cdot(\ln w)^{B} \cdot \lambda^{-\rho} w^{\beta_{1}(\bar{\mu})+b_{1}-1} \\
& c_{s}(\lambda, w) \exp \left(a_{\mu(1)} \cdot \frac{w^{D}}{\lambda}\right) d w
\end{aligned}
$$


Computation of $c_{s}(\lambda ; w)$.

We assume here that $0<\alpha<1$.

$$
c_{s}(\lambda ; w)=\left(f_{\lambda}\right)_{s, s}(w ; 0)=\frac{(-1)^{s}}{(s-1) !} \int_{0}^{\infty} \tau^{s-1}\left(f_{\lambda}\right)_{s}(w, \tau) d \tau
$$

where according to (14) and (20).

$$
\begin{aligned}
\left(f_{\lambda}\right)_{s}(w, \tau) & =f_{\lambda}(w, \tau)-\left[\frac{a_{0}(\lambda ; w)}{\tau^{\alpha}}+\ldots+\frac{a_{s-1}(\lambda ; w)}{\tau^{s-1+\alpha}}\right](\ln \tau)^{k} \\
& =\frac{(\ln \tau)^{k}}{\tau^{\alpha+c^{-}}}\left[\exp \left(\frac{a_{\nu}}{\lambda} w^{\beta_{1}(\nu)} \tau^{\beta_{2}(\nu)}\right)-\sum_{i \in \mathbf{N}, i<\bar{r}} \frac{1}{i !}\left(\frac{a_{\nu}}{\lambda} w^{\beta_{1}(\nu)} \tau^{\beta_{2}(\nu)}\right)^{i}\right]
\end{aligned}
$$

with $\bar{r}=\frac{s-c^{-}}{-\beta_{2}(\nu)}$.

$$
\begin{aligned}
c_{s}(\lambda ; w)= & \frac{(-1)^{s}}{(s-1) !} \int_{0}^{\infty} \frac{(\ln \tau)^{k} \tau^{s-1}}{\tau^{\alpha+c^{-}}} \\
& {\left[\exp \left(\frac{a_{\nu}}{\lambda} w^{\beta_{1}(\nu)} \tau^{\beta_{2}(\nu)}\right)-\sum_{0 \leq i<\bar{r}} \frac{1}{i !}\left(\frac{a_{\nu}}{\lambda} w^{\beta_{1}(\nu)} \tau^{\beta_{2}(\nu)}\right)^{i}\right] d \tau }
\end{aligned}
$$

Set $u=\frac{1}{\lambda} w^{\beta_{1}(\nu)} \tau^{\beta_{2}(\nu)}$, where $\beta_{2}(\nu)<0, w$ stated.

$$
\begin{aligned}
c_{s}(\lambda ; w)= & \frac{(-1)^{s}}{(s-1) !}\left(\frac{w^{\beta_{1}(\nu)}}{\lambda}\right)^{\frac{s-c^{-}-\alpha}{-\beta_{2}(\nu)}}\left(\frac{-1}{\beta_{2}(\nu)}\right) \int_{0}^{\infty}\left(\frac{1}{\beta_{2}(\nu)} \ln \left[\frac{\lambda u}{w^{\beta_{1}(\nu)}}\right]\right)^{k} u^{\frac{s-c^{-}-\alpha}{\beta_{2}(\nu)}-1} \\
& {\left[\exp \left(a_{\nu} u\right)-\sum_{0 \leq i<\bar{r}} \frac{1}{i !}\left(a_{\nu} u\right)^{i}\right] d u }
\end{aligned}
$$

There are constants $d_{i, j}^{\prime}$ such that

$$
c_{s}(\lambda ; w)=(-1)^{s}\left(\frac{w^{\beta_{1}(\nu)}}{\lambda}\right)^{\bar{r}+\frac{\alpha}{\beta_{2}(\nu)}}\left[\sum_{i+j \leq k} d_{i, j}^{\prime}(\ln \lambda)^{i}(\ln w)^{j}\right]
$$

Thus

$$
\begin{aligned}
I_{2}= & \sum_{s=1}^{n} \sum_{\rho} \sum_{\bar{\mu}, \beta_{2}(\bar{\mu})=s-c^{+}-1} h_{s-1}(\rho, \bar{\mu}) \int_{\mathbb{R}_{+}}\left(\frac{w^{\beta_{1}(\nu)}}{\lambda}\right)^{\bar{r}+\frac{\alpha}{\beta_{2}(\nu)}}(\ln w)^{B_{1}} \lambda^{-\rho} w^{\beta_{1}(\bar{\mu})+b_{1}-1} \\
& {\left[\sum_{i+j \leq k} d_{i, j}^{\prime}(\ln \lambda)^{i}(\ln w)^{j}\right] \exp \left(\frac{1}{\lambda} a_{\mu(1)} w^{D}\right) d w }
\end{aligned}
$$

It is then sufficient to put $w^{\prime}=w \lambda^{-1 / D}$ to obtain the expansion expected.

Remark. The study of $I_{2}$ in the case $\alpha=1$ is dealt exactly in the same way. 
4.3.3. Study of $I_{3}$. Assume $0<\alpha<1$.

$$
\begin{gathered}
I_{3}=\int_{\mathbb{R}_{+}}<\left(f_{\lambda}\right)_{n} ; \phi_{\lambda}>d w \\
\left.<\left(f_{\lambda}\right)_{n} ; \phi_{\lambda}>=(-1)^{n} \int_{\mathbb{R}_{+}}\left(f_{\lambda}\right)_{n, n}(w, t) \frac{\partial^{n} \phi_{\lambda}}{\partial t^{n}}(w, t) d t \quad \text { (for any } w\right)
\end{gathered}
$$

where

$$
\begin{gathered}
\left(f_{\lambda}\right)_{n, n}(w, t)=\frac{(-1)^{n}}{(n-1) !} \int_{t}^{+\infty}\left(f_{\lambda}\right)_{n}(w, \tau)(\tau-t)^{n-1} d \tau \\
<\left(f_{\lambda}\right)_{n} ; \phi_{\lambda}>=\frac{1}{(n-1) !} \int_{0}^{\infty}\left[\int_{t}^{\infty}\left(f_{\lambda}\right)_{n}(w, \tau)(\tau-t)^{n-1} d \tau\right] \cdot \frac{\partial^{n} \phi_{\lambda}}{\partial t^{n}}(w, t) d t
\end{gathered}
$$

from $(37)$

$$
\begin{aligned}
& f_{\lambda}(w, \tau)=\frac{(\ln \tau)^{k}}{\tau^{\alpha+c^{-}}} \cdot \exp \left(\frac{a_{\nu}}{\lambda} w^{\beta_{1}(\nu)} \tau^{\beta_{2}(\nu)}\right) \\
& =\sum_{0 \leq i<\bar{R}} \frac{1}{i !}\left(\frac{a_{\nu}}{\lambda} \cdot w^{\beta_{1}(\nu)}\right)^{i} \tau^{-\alpha+i\left(\beta_{2}(\nu)\right)-c^{-}} \cdot(\ln \tau)^{k}+\left(f_{\lambda}\right)_{n}(w, \tau)
\end{aligned}
$$

where we put $\bar{R}=\frac{n-c^{-}}{-\beta_{2}(\nu)}$. We consider $n \in \mathbb{N}$ such that $\bar{R} \in \mathbb{N}$.

Moreover, since $a_{\nu}<0$

$$
\left|\exp \left(\frac{a_{\nu}}{\lambda} w^{\beta_{1}(\nu)} \tau^{\beta_{2}(\nu)}\right)-\sum_{0 \leq i<\bar{R}} \frac{1}{i !}\left(\frac{a_{\nu}}{\lambda} \cdot w^{\beta_{1}(\nu)} \tau^{\beta_{2}(\nu)}\right)^{i}\right| \leq\left|\left(\frac{a_{\nu}}{\lambda} w^{\beta_{1}(\nu)} \tau^{\beta_{2}(\nu)}\right)\right|^{\bar{R}}
$$

hence

$$
\begin{aligned}
\left|\left(f_{\lambda}\right)_{n}(w ; \tau)\right| & \leq \frac{|\ln \tau|^{k}}{\tau^{\left(\alpha+c^{-}\right)}}\left|\left(\frac{a_{\nu}}{\lambda} w^{\beta_{1}(\nu)} \tau^{\beta_{2}(\nu)}\right)\right|^{\bar{R}}=\frac{|\ln \tau|^{k}}{\tau^{(\alpha+n)}}\left|\left(\frac{a_{\nu}}{\lambda} w^{\beta_{1}(\nu)}\right)\right|^{\bar{R}} \\
\left|<\left(f_{\lambda}\right)_{n} ; \phi_{\lambda}>\right| & \leq \mathrm{const}\left|\int_{0}^{\infty} \int_{t}^{\infty} \frac{|\ln \tau|^{k}}{\tau^{\alpha+n}} \frac{w^{\bar{R} \beta_{1}(\nu)}}{\lambda^{\bar{R}}} \cdot(\tau-t)^{n-1} d \tau \cdot\right| \frac{\partial^{n} \phi_{\lambda}}{\partial t^{n}}(w, t)|d t| \\
& \leq \text { const. } \frac{w^{\bar{R} \beta_{1}(\nu)}}{\lambda^{\bar{R}}} \int_{0}^{\infty} \int_{t}^{\infty}\left|(\ln \tau)^{k} \frac{\tau^{n-1}}{\tau^{\alpha+n}} d \tau \cdot \frac{\partial^{n} \phi_{\lambda}}{\partial t^{n}}(w, t)\right| d \tau d t \\
& =\text { const. } \frac{w^{\bar{R} \beta_{1}(\nu)}}{\lambda^{\bar{R}}} \int_{0}^{\infty} \int_{t}^{\infty}\left|\frac{(\ln \tau)^{k}}{\tau^{\alpha+1}} d \tau \cdot \frac{\partial^{n} \phi_{\lambda}}{\partial t^{n}}(w, t)\right| d \tau d t
\end{aligned}
$$

where $\int_{\infty}^{t} \frac{(\ln \tau)^{k}}{\tau^{\alpha+1}} d \tau=F_{k, 1, \alpha}(t)$ (see (15) with $s=1$ )

And since $F_{k, 1, \alpha}(t)=\frac{1}{t^{\alpha}} \cdot \sum_{r=0}^{k}$ const. $(\ln t)^{r}$ to study $\left|<\left(f_{\lambda}\right)_{n} ; \phi_{\lambda}>\right|$ means to study expressions :

$$
\frac{w^{\bar{R} \beta_{1}(\nu)}}{\lambda^{\bar{R}}} \int_{0}^{\infty} \frac{1}{t^{\alpha}}\left|(\ln t)^{r} \cdot \frac{\partial^{n} \phi_{\lambda}}{\partial t^{n}}(w, t)\right| d t \quad \text { for } 0 \leq r \leq k
$$


Integrating with respect to $w$ gives :

$$
\int_{\mathbb{R}_{+}^{2}} \frac{w^{\bar{R} \beta_{1}(\nu)}}{\lambda^{\bar{R}}}\left|(\ln t)^{r} \cdot \frac{\partial^{n} \phi_{\lambda}}{\partial t^{n}}(w, t)\right| \cdot \frac{d t}{t^{\alpha}} d w
$$

From (26) it means that :

$$
\begin{aligned}
\left|I_{3}\right| \leq & \sum_{\text {finite }} \text { const. } \int_{\mathbb{R}_{+}^{2}}|(\ln w)|^{B_{1}}|(\ln t)|^{r} \cdot \exp \left(\frac{1}{\lambda} \sum_{\mu \in \Delta \backslash\{\nu\}} a_{\mu} w^{\beta_{1}(\mu)} t^{\beta_{2}(\mu)}\right) \\
& \cdot \frac{1}{\lambda^{\rho+\bar{R}}} \cdot w^{\beta_{1}(\bar{\mu})+\bar{R} \cdot \beta_{1}(\nu)} \cdot w^{b_{1}-1} \cdot t^{\beta_{2}(\bar{\mu})} t^{c^{+}-n-\alpha} d w d t
\end{aligned}
$$

where $\bar{\mu} \in \mathcal{S}_{\rho}, \beta_{2}(\bar{\mu}) \geq n-c^{+}$, and coming back to $x$ as in (31) and (33) we obtain

$$
\left|I_{3}\right| \leq \sum_{\text {finite }} \text { const. } \frac{1}{\lambda^{\rho+\bar{R}}} \int_{\mathbb{R}_{+}^{2}} x^{\bar{\mu}}|(\ln x)|^{\Sigma} x^{\bar{R} \nu} \cdot x^{b^{\prime}-1} \cdot \exp \left(\frac{P_{\Delta \backslash\{\nu\}}(x)}{\lambda}\right) d x
$$

where $P_{\Delta \backslash\{\nu\}}(x)=P^{*}(x)$ of Newton diagram $\Delta^{*}$. We need an order of magnitude of each of these integrals. According to the remark following Lemma 4, (i) and (ii) of Lemma 4 is valid for

$$
L^{\prime}(\theta, \gamma, P)=\int_{\mathbb{R}_{+}^{2}} x^{\theta-1}|\ln x|^{\gamma} \exp \left(\frac{1}{\lambda} P(x)\right) d x
$$

i.e. $L^{\prime}(\theta, \gamma, P)=o\left(\lambda^{A_{\Delta}(\theta)-\varepsilon}\right)$ as $\lambda \rightarrow 0^{+}$, for any $\varepsilon>0$, if $(\theta, \gamma, P)$ satisfies the HP hypothesis.

To apply this here, we must just check that $\tilde{\mu}=\bar{\mu}+\bar{R} \cdot \nu+b^{\prime}$ belongs to $\operatorname{int} \mathcal{C}\left(\Delta^{*}\right)$, i.e. that $\beta_{2}(\tilde{\mu})>0$.

$$
\begin{aligned}
& \beta_{2}(\tilde{\mu})=\operatorname{det}(\mu(1), \tilde{\mu})=\beta_{2}\left(b^{\prime}\right)+\beta_{2}(\bar{\mu})+\bar{R} \cdot \beta_{2}(\nu) \\
& \geq \beta_{2}\left(b^{\prime}\right)+n-c^{+}+\bar{R} \cdot \beta_{2}(\nu)=\beta_{2}\left(b^{\prime}\right)+n-c^{+}-n+c^{-} \\
& =b_{2}-c=1-\alpha>0 \\
& \text { It remains to evaluate } A_{\Delta^{*}}(\tilde{\mu}) .
\end{aligned}
$$

$\tilde{\mu}=\bar{\mu}+b^{\prime}+\bar{R} . \nu$ thus $A_{\Delta^{*}}(\tilde{\mu}) \geq A_{\Delta^{*}}(\bar{\mu})+A_{\Delta^{*}}\left(b^{\prime}\right)+\bar{R} \cdot A_{\Delta^{*}}(\nu) \geq \rho+A_{\Delta^{*}}\left(b^{\prime}\right)+\bar{R} \cdot A_{\Delta^{*}}(\nu)$ because $A_{\Delta^{*}}(\bar{\mu}) \geq \sum_{1}^{\rho} A_{\Delta^{*}}(\bar{\mu}(i)) \geq \rho$.

Then $A_{\Delta^{*}}(\tilde{\mu}) \geq \rho+A_{\Delta^{*}}\left(b^{\prime}\right)+\bar{R} \cdot A_{\Delta^{*}}(\nu)$

Hence finally $I_{3}$ satisfies, for $\lambda$ small enough, for any $\varepsilon>0$ :

$$
\begin{aligned}
\left|I_{3}\right| & =\left|\int_{\mathbb{R}_{+}}<\left(f_{\lambda}\right)_{n} ; \phi_{\lambda}>d w\right| \leq \sum_{\text {finite }} \text { const. } \lambda^{-\rho-\bar{R}} \lambda^{A_{\Delta^{*}}(\tilde{\mu})-\varepsilon} \\
& \leq \text { const. } \lambda^{A_{\Delta^{*}}\left(b^{\prime}\right)+\bar{R} .\left(A_{\Delta^{*}}(\nu)-1\right)-\varepsilon}
\end{aligned}
$$

where $A_{\Delta^{*}}(\nu)>1$. Thus $I_{3}$ is $o\left(\lambda^{M}\right)$ for $n$ large enough. More precisely, $I_{3}=o\left(\lambda^{M}\right)$ as soon as $A_{\Delta^{*}}\left(b^{\prime}\right)+\bar{R} \cdot\left(A_{\Delta^{*}}(\nu)-1\right)>M$ i.e. with $\bar{R}>\frac{M-A_{\Delta^{*}}\left(b^{\prime}\right)}{A_{\Delta^{*}}(\nu)-1}, \bar{R} \in \mathbb{N}$

It is then sufficient to take

$$
n=c^{-}+\left(-\beta_{2}(\nu)\right) \bar{R} \text { where } \bar{R} \text { is the integer part of } \frac{M-A_{\Delta^{*}}\left(b^{\prime}\right)}{A_{\Delta^{*}}(\nu)-1}+1
$$

The case $\alpha=1$ must be dealt in the same way.

This ends the proof of Proposition 6. 


\section{Proof of Proposition 7.}

$L(\theta, \gamma, P)=\int_{\mathbb{R}_{+}^{p}} \exp \left(\frac{1}{\lambda} \sum_{\mu \in \Delta} a_{\mu} x^{\mu}\right) x^{\theta-1}(\ln x)^{\gamma} d x$ where $\Delta$ is just the facet $F$. We introduce the change of variables $x \mapsto v^{(\sigma)}$ where $\sigma$ is a facet-system of $p$ independent vertices of $F$. From Lemma 5:

$$
L(\theta, \gamma, P)=\int_{\mathbb{R}_{+}^{p}} \exp \left(\frac{1}{\lambda} \sum_{\mu \in F} a_{\mu} v^{\beta(\mu)}\right) \cdot v^{\beta(\theta)-1} \cdot D^{p-1} \prod_{i=1}^{p}\left(\ln v^{\beta\left(e_{i}\right)}\right)^{\gamma_{i}} d v
$$

and setting $u_{i}=\lambda^{-\frac{1}{D}} v_{i}$ as in the proof of Lemma 4 , we obtain:

$$
\begin{aligned}
L(\theta, \gamma, P)= & \lambda^{A_{F}(\theta)} \int_{\mathbb{R}_{+}^{p}} \exp \left(\sum_{\mu \in F} a_{\mu} \lambda^{A_{F}(\mu)-1} u^{\beta(\mu)}\right) \cdot u^{\beta(\theta)-1} \\
& \prod_{i=1}^{p}\left(\ln \lambda^{A_{F}\left(e_{i}\right)} v^{\beta\left(e_{i}\right)}\right)^{\gamma i} \cdot D^{p-1} d u \\
= & \lambda^{A_{F}(\theta)} \sum_{k_{1}=0}^{\gamma_{1}} \ldots \sum_{k_{p}=0}^{\gamma_{p}}\left(\begin{array}{c}
\gamma_{1} \\
k_{1}
\end{array}\right) \cdot \cdot\left(\begin{array}{c}
\gamma_{p} \\
k_{p}
\end{array}\right) \cdot \prod_{i=1}^{p}\left(\ln \lambda^{A_{F}\left(e_{i}\right)}\right)^{k_{i}} \\
& \int_{\mathbb{R}_{+}^{p}} \exp \left(\sum_{\mu \in F} a_{\mu} u^{\beta(\mu)}\right) \cdot u^{\beta(\theta)-1} \cdot \prod_{i=1}^{p}\left(\ln u^{\beta\left(e_{i}\right)}\right)^{\gamma_{i}-k_{i}} \cdot D^{p-1} d u
\end{aligned}
$$

since $A_{F}(\mu)=1$ for $\mu \in F$.

\section{Study of an example.}

6.1. Introduction. Throughout this section, to show the working of our algorithm, we will follow a simple example of an integral of the type $J_{H, \chi}(\lambda)$. For this example, we take $H$ from $\mathbb{R}^{2}$ to $\mathbb{R}$, defined by:

$$
H\left(x_{1}, x_{2}\right)=-\left(x_{1}^{6}+x_{1}^{2} x_{2}^{2}+x_{2}^{6}+x_{1}^{4} x_{2}+x_{1} x_{2}^{4}+x_{1}^{3} x_{2}^{4}\right)
$$

and $\chi$ smooth of compact support $K$, such that

$$
\chi\left(x_{1}, x_{2}\right)=x_{1}^{2} x_{2}^{4}+x_{1}^{7} x_{2}^{3} \quad \text { in a neighborhood of } 0
$$

We want to compute the asymptotic expansion of order $M=\frac{5}{2}$ of $J_{H, \chi}(\lambda)$.

The principal part of $H$ is

$$
\hat{H}(x)=-\left(x_{1}^{6}+x_{1}^{2} x_{2}^{2}+x_{2}^{6}+x_{1}^{4} x_{2}+x_{1} x_{2}^{4}\right)
$$

The Newton diagram $\Delta$ of $H$ has two facets $F_{1}$ and $F_{2}$ with

$$
A_{F_{1}}(\mu)=\frac{1}{3} \mu_{1}+\frac{1}{6} \mu_{2} \text { and } A_{F_{2}}(\mu)=\frac{1}{6} \mu_{1}+\frac{1}{3} \mu_{2}
$$

$\mu \in F_{1} \Rightarrow A_{F_{1}}(\mu)=1$, and $\mu \in F_{2} \Rightarrow A_{F_{2}}(\mu)=1$. Moreover

$$
A_{\Delta}(\mu)=\inf _{F_{i}}\left[A_{F_{i}}(\mu)\right]
$$




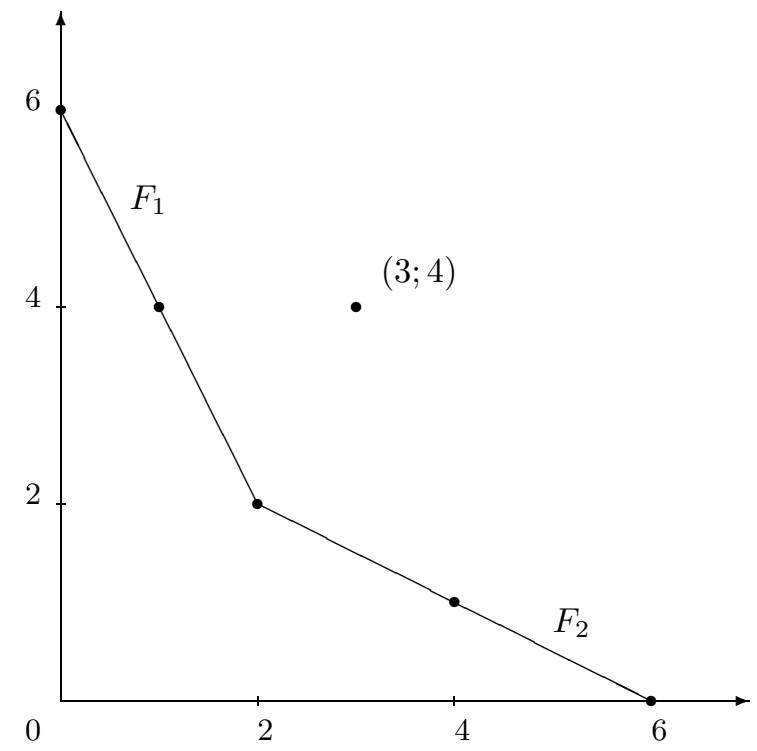

Newton polygon of $H$

According to Proposition 1

$$
\int \chi(x) \cdot \exp (H(x) / \lambda) d x=\int_{B(\delta)} \chi(x) \cdot \exp (H(x) / \lambda) d x+O\left(e^{-d / \lambda}\right)
$$

6.2. Approximation of the amplitude. With our example, to apply Proposition 2 , we must evaluate $A_{\Delta}(\theta)$ for $\theta=(2 ; 4)+(1 ; 1)=(3 ; 5)$ and $\theta=(7 ; 3)+(1 ; 1)=$ $(8 ; 4)$.

$$
\begin{aligned}
& A_{\Delta}(3 ; 5)=\inf _{F_{i}}\left[A_{F_{i}}(3 ; 5)\right]=\inf \left[\frac{11}{6}, \frac{13}{6}\right]=\frac{11}{6}<\frac{5}{2} \\
& A_{\Delta}(8 ; 4)=\inf _{F_{i}}\left[A_{F_{i}}(8 ; 4)\right]=\inf \left[\frac{10}{3}, \frac{8}{3}\right]=\frac{8}{3}>\frac{5}{2} \\
& \text { Proposition } 2 \text { gives then, for } M=\frac{5}{2}
\end{aligned}
$$

$$
\int \chi(x) \cdot \exp (H(x) / \lambda) d x=\int_{B(\delta)} x_{1}^{2} x_{2}^{4} \cdot \exp (H(x) / \lambda) d x+o\left(\lambda^{\frac{5}{2}}\right)
$$

6.3. Reduction of the phase. Now, we want to apply Proposition 3 to our example. It will allow to simplify the phase, putting $\hat{H}$ instead of $H$.

$\eta_{0}>0$ is such that there is no point of the support of $H$ strictly between $\Delta$ and $\left(1+\eta_{0}\right) \Delta$. More precisely, $\eta_{0}=\inf \left(A_{\Delta}(\mu)-1 ; \mu \in \Sigma(H) \backslash \Delta(H)\right)$.

$(3 ; 4)$ is the only point of $\Sigma(H) \backslash \Delta$. One finds $A_{\Delta}(3 ; 4)=\inf _{F_{i}}\left[A_{F_{i}}(3 ; 4)\right]=$ $\inf \left[\frac{5}{3}, \frac{11}{6}\right]=\frac{5}{3}$. So, we can take $\eta_{0}=\frac{2}{3}$. As $M=\frac{5}{2}$ and $\theta=(2 ; 4)+(1 ; 1)=(3 ; 5)$, we have $\frac{M-A_{\Delta}(\theta)}{\eta_{0}}=\frac{\frac{5}{2}-\frac{11}{6}}{\frac{2}{3}}=1$, so we set $M^{\prime}=1$. Proposition 3 gives then $R_{1}(x)=$ $-x_{1}^{3} x_{2}^{4}$, and

$$
\int \chi(x) \cdot \exp (H(x) / \lambda) d x=\int_{B(\delta)} x_{1}^{2} x_{2}^{4} \cdot\left[1-\frac{x_{1}^{3} x_{2}^{4}}{\lambda}\right] \exp (\hat{H}(x) / \lambda) d x+o\left(\lambda^{\frac{5}{2}}\right)
$$


6.4. Extension of the domain. Proposition 4 gives

$$
\int \chi(x) \cdot \exp (H(x) / \lambda) d x=\int_{\mathbb{R}^{2}} x_{1}^{2} x_{2}^{4} \cdot\left[1-\frac{x_{1}^{3} x_{2}^{4}}{\lambda}\right] \exp \left(\frac{\hat{H}(x)}{\lambda}\right) d x+o\left(\lambda^{\frac{5}{2}}\right)
$$

6.5. Simplification of facets. We must then apply Proposition 5 to $\int_{\mathbb{R}^{p}} x_{1}^{2} x_{2}^{4} \exp \left(\frac{\hat{H}(x)}{\lambda}\right) d x$ with $M=5 / 2$ and to $\int_{\mathbb{R}^{p}} x_{1}^{5} x_{2}^{8} \exp \left(\frac{\hat{H}(x)}{\lambda}\right) d x$ with $M=7 / 2$, i.e. for $\theta=(3 ; 5)$ and $\theta=(6 ; 9)$.

We have here $S=\{(6 ; 0),(2 ; 2),(0 ; 6)\}$ and $\Sigma(\hat{H})=S \cup\{(1 ; 4),(4 ; 1)\}$

We apply Proposition 5 with $B=\{(1 ; 4),(4 ; 1)\}, \Delta_{1}=B \cap\left(F_{1} \backslash F_{2}\right)=\{(1 ; 4)\}$, $\Delta_{2}=B \cap\left(F_{2} \backslash F_{1}\right)=\{(4 ; 1)\}, B_{1}=\Delta_{2}=\{(4 ; 1)\}, B_{2}=\Delta_{1}=\{(1 ; 4)\}, B_{0}=\emptyset$.

$\eta_{1}=\inf \left(A_{F_{2}}(\mu)-1 ; \mu \in \Delta_{1}\right)=A_{F_{2}}(1 ; 4)-1=\frac{3}{2}-1=\frac{1}{2}$

$\eta_{2}=\inf \left(A_{F_{1}}(\mu)-1 ; \mu \in \Delta_{2}\right)=A_{F_{1}}(4 ; 1)-1=\frac{3}{2}-1=\frac{1}{2}$ which implies that $\eta=\frac{1}{2}$.

$$
\begin{gathered}
\int_{\mathbb{R}^{2}} x^{\theta-1} \cdot \exp \left(\frac{H_{S \cup B}(x)}{\lambda}\right) d x \\
=o\left(\lambda^{\frac{5}{2}}\right)+\sum_{k_{1}=0}^{M^{\prime \prime}} \frac{1}{\lambda^{k_{1}}} \frac{1}{k_{1} !} \int_{\mathbb{R}^{2}} x^{\theta-1}\left(-x_{1} x_{2}^{4}\right)^{k_{1}} \exp \left(\frac{H_{S \cup B_{1}}(x)}{\lambda}\right) d x \\
+\sum_{k_{2}=0}^{M^{\prime \prime}} \frac{1}{\lambda^{k_{2}}} \frac{1}{k_{2} !} \int_{\mathbb{R}^{2}} x^{\theta-1}\left(-x_{1}^{4} x_{2}\right)^{k_{2}} \exp \left(\frac{H_{S \cup B_{2}}(x)}{\lambda}\right) d x \\
-\sum_{k_{1}=0}^{M^{\prime \prime}} \sum_{k_{2}=0}^{M^{\prime \prime}} \frac{1}{\lambda^{k_{1}+k_{2}}} \frac{1}{k_{1} ! k_{2} !} \int_{\mathbb{R}^{2}} x^{\theta-1}\left(-x_{1} x_{2}^{4}\right)^{k_{1}}\left(-x_{1}^{4} x_{2}\right)^{k_{2}} \exp \left(\frac{H_{S \cup B_{0}}(x)}{\lambda}\right) d x
\end{gathered}
$$

where $M$ " is the integer part of $\frac{1}{\eta}\left(M-A_{\Delta}(\theta)\right)=2\left(M-A_{\Delta}(\theta)\right)$

1) $\theta=(3 ; 5)$ and $M=\frac{5}{2}$ leads to $A_{\Delta}(\theta)=\frac{11}{6}$ so $M$ " is the integer part of $5-2\left(\frac{11}{6}\right)=\frac{4}{3}$ i.e. $M^{\prime \prime}=1$

2) $\theta=(6 ; 9)$ and $M=\frac{7}{2}$ leads to $A_{\Delta}(\theta)=\inf \left(\frac{7}{2} ; 4\right)=\frac{7}{2}$ so $M$ " is the integer part of $7-2\left(\frac{7}{2}\right)=0$ i.e. $M "=0$.

Finally

$$
\begin{gathered}
\int_{\mathbb{R}^{2}} x_{1}^{2} x_{2}^{4} \cdot\left[1-\frac{x_{1}^{3} x_{2}^{4}}{\lambda}\right] \exp \left(\frac{\hat{H}(x)}{\lambda}\right) d x \\
=o\left(\lambda^{\frac{5}{2}}\right)+\int_{\mathbb{R}^{2}} x_{1}^{2} x_{2}^{4}\left(1-\frac{x_{1} x_{2}^{4}}{\lambda}\right) \cdot \exp \left(\frac{H_{S \cup B_{1}}(x)}{\lambda}\right) d x \\
+\int_{\mathbb{R}^{2}} x_{1}^{2} x_{2}^{4}\left(1-\frac{x_{1}^{4} x_{2}}{\lambda}\right) \cdot \exp \left(\frac{H_{S \cup B_{2}}(x)}{\lambda}\right) d x \\
-\int_{\mathbb{R}^{2}} x_{1}^{2} x_{2}^{4} \cdot\left(1-\frac{x_{1}^{4} x_{2}}{\lambda}-\frac{x_{1} x_{2}^{4}}{\lambda}+\frac{x_{1}^{5} x_{2}^{5}}{\lambda^{2}}\right) \exp \left(\frac{H_{S}(x)}{\lambda}\right) d x \\
-\frac{1}{\lambda} \int_{\mathbb{R}^{2}} x_{1}^{5} x_{2}^{8} \cdot \exp \left(\frac{H_{S \cup B_{1}}(x)}{\lambda}\right) d x-\frac{1}{\lambda} \int_{\mathbb{R}^{2}} x_{1}^{5} x_{2}^{8} \cdot \exp \left(\frac{H_{S \cup B_{2}}(x)}{\lambda}\right) d x \\
+\frac{1}{\lambda} \int_{\mathbb{R}^{2}} x_{1}^{5} x_{2}^{8} \cdot \exp \left(\frac{H_{S}(x)}{\lambda}\right) d x
\end{gathered}
$$


We have then 11 integrals of the same type to evaluate. Henceforth, we will limit our example to the study of the simplest one (on $\mathbb{R}_{+}^{2}$ ), i.e.

$$
L_{1}=\int_{\mathbb{R}_{+}^{2}} x_{1}^{2} x_{2}^{4} \cdot \exp \left(-\frac{1}{\lambda}\left(x_{1}^{6}+x_{1}^{2} x_{2}^{2}+x_{2}^{6}\right)\right) d x
$$

\subsection{Suppression of vertices.}

6.6.1. First step. We have

$$
L_{1}=L\left(b^{\prime}, b^{\prime \prime}, P\right)=\int_{\mathbb{R}_{+}^{2}} x^{b^{\prime}-1}(\ln x)^{b^{\prime \prime}} \exp \left(\frac{P(x)}{\lambda}\right) d x
$$

with $b^{\prime}=(3 ; 5), b "=(0 ; 0), P(x)=-\left(x_{1}^{6}+x_{1}^{2} x_{2}^{2}+x_{2}^{6}\right)$

i.e. $L_{1}=L((3 ; 5),(0 ; 0), P)$

We have $A_{\Delta}\left(b^{\prime}\right)=A_{F_{1}}\left(b^{\prime}\right)=\frac{11}{6}$ and $A_{F_{2}}\left(b^{\prime}\right)=\frac{13}{6}$.

We want to remove the vertex $\nu=(6 ; 0)$. Let $\mu(1)=(2 ; 2)$ and $\mu(2)=(0 ; 6)$.

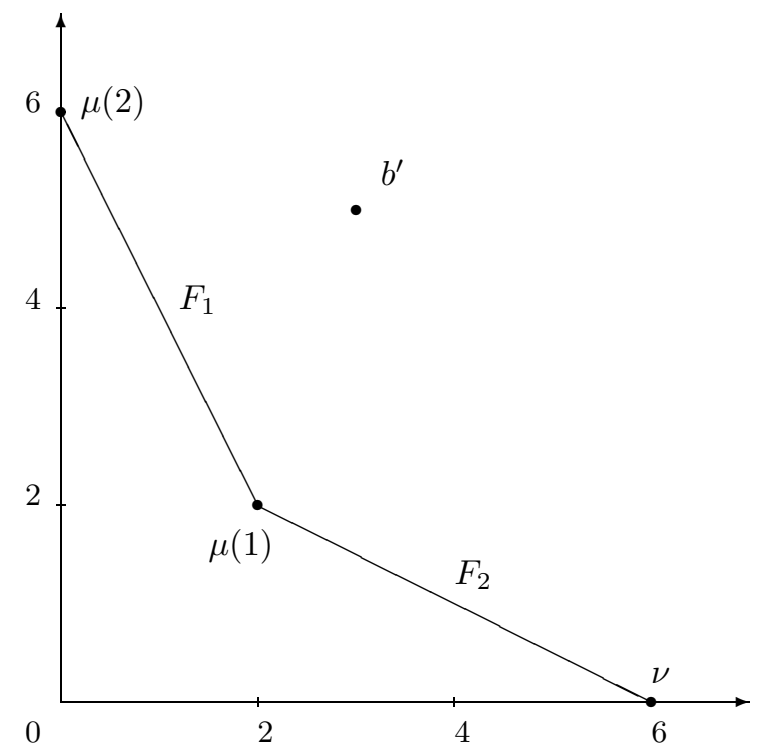

Newton diagram of $P$

According to Lemma 5, the change of variables associated to the facet system $(\mu(1), \mu(2))$ is given by $x^{\mu}=v_{1}^{\beta_{1}(\mu)} v_{2}^{\beta_{2}(\mu)}$, where $\beta_{1}(\mu)=\operatorname{det}(\mu, \mu(2))=6 \mu_{1}$ and $\beta_{2}(\mu)=\operatorname{det}(\mu(1), \mu)=2\left(\mu_{2}-\mu_{1}\right)$, i.e. $x^{\mu}=v_{1}^{6 \mu_{1}} v_{2}^{2\left(\mu_{2}-\mu_{1}\right)}$.

In particular, $v_{1}^{12}=x_{1}^{2} x_{2}^{2}, v_{2}^{12}=x_{2}^{6}$ and $x^{b^{\prime}}=x_{1}^{3} x_{2}^{5}=v_{1}^{18} v_{2}^{4}$ We obtain with $D=\operatorname{det}(\mu(1), \mu(2))=12$

$$
L_{1}=12 M_{B}
$$

where 


$$
M_{B}=\int_{\mathbb{R}_{+}^{2}} v_{1}^{17} v_{2}^{3} \exp \left(-\frac{1}{\lambda}\left[v_{1}^{12}+v_{2}^{12}+v_{1}^{36} v_{2}^{-12}\right]\right) d v
$$

$b_{2}=4$ so (11) becomes here $\alpha=1$ and $c=4$ (i.e. $c^{+}=4, c^{-}=0$ ). We set $w=v_{1}$, $t=v_{2}$. Equation (12) is now

$$
\begin{aligned}
M_{B} & =\int_{\mathbb{R}_{+}^{2}} t^{4} w^{17} \exp \left(-\frac{1}{\lambda}\left[w^{12}+t^{12}\right]\right) \cdot \frac{1}{t} \exp \left(-\frac{1}{\lambda}\left[w^{36} t^{-12}\right]\right) d w d t \\
& =\int_{\mathbb{R}_{+}^{2}} \phi_{\lambda}(w ; t) \cdot f_{\lambda}(w ; t) d w d t
\end{aligned}
$$

where following (19), $\phi_{\lambda}$ and $f_{\lambda}$ are defined by

$$
\begin{aligned}
& \phi_{\lambda}(w ; t)=t^{4} w^{17} \exp \left(-\frac{1}{\lambda}\left[w^{12}+t^{12}\right]\right) \\
& f_{\lambda}(w ; t)=\frac{1}{t} \exp \left(-\frac{1}{\lambda}\left[w^{36} t^{-12}\right]\right)
\end{aligned}
$$

6.6.2. Second step. According to $(20), f_{\lambda}(w ; t)$ has the asymptotic expansion as $t \rightarrow+\infty$

$$
f_{\lambda}(w ; t)=\frac{1}{t} \exp \left(-\frac{1}{\lambda}\left[w^{36} t^{-12}\right]\right) \sim \frac{1}{t} \sum_{\bar{r}=0}^{\infty} \frac{1}{\bar{r} !}\left(-\frac{1}{\lambda} \cdot \frac{w^{36}}{t^{12}}\right)^{\bar{r}}
$$

and

$$
f_{\lambda}(w ; t) \sim \sum_{s=0}^{\infty} \frac{a_{s}(\lambda, w)}{t^{s+1}}
$$

so

$$
\begin{aligned}
& a_{s}(\lambda, w)=\frac{1}{\bar{r} !}\left(-\frac{1}{\lambda} \cdot w^{36}\right)^{\bar{r}} \text { for } s=12 \bar{r}, \text { with } \bar{r} \in \mathbb{N} \\
& a_{s}(\lambda, w)=0 \text { else }
\end{aligned}
$$

Moreover we have a decomposition $M_{B}=I_{1}+I_{2}+I_{3}$ as in (22).

6.6.3. Third step. We take $n=c^{-}+\left(-\beta_{2}(\nu)\right) \cdot \bar{R}=12 \bar{R}$, where $\bar{R}$ is the integer part of $\frac{M-A_{\Delta^{*}}\left(b^{\prime}\right)}{A_{\Delta^{*}}(\nu)-1}+1$.

Here $\Delta^{*}=F_{1}$, since if we remove $\nu$, the facet $F_{2}$ disappears. So

$$
\frac{M-A_{\Delta^{*}}\left(b^{\prime}\right)}{A_{\Delta^{*}}(\nu)-1}=\frac{\frac{5}{2}-\frac{11}{6}}{2-1}=\frac{2}{3}
$$

thus $\bar{R}=1$ and $n=12$. 
Study of $I_{1}$.

$$
I_{1}=\int_{\mathbb{R}_{+}}\left[\sum_{s=0}^{11} a_{s}(\lambda, w)<(\ln t)_{+}^{k} t_{+}^{-s-\alpha} ; \phi_{\lambda}>\right] d w
$$

where $k=0$ and $\alpha=1$.

By definition $<t_{+}^{-s-1} ; \phi_{\lambda}>=\frac{-1}{s !} \int_{0}^{+\infty} \ln t \cdot \frac{\partial^{s+1} \varphi_{\lambda}}{\partial t^{s+1}}(w ; t) d t$.

But $a_{0}(\lambda, w)=1$ and $a_{s}(\lambda, w)=0$ if $1 \leq s \leq 11$.

So

$$
\begin{aligned}
I_{1} & =\int_{\mathbb{R}_{+}}<t_{+}^{-1} ; \phi_{\lambda}>d w \\
& =-\int_{\mathbb{R}_{+}^{2}} w^{17} \exp \left(-\frac{1}{\lambda} w^{12}\right) \cdot \ln t \cdot \frac{\partial}{\partial t}\left(t^{4} \exp \left(-\frac{1}{\lambda} t^{12}\right)\right) d t d w \\
& =\int_{\mathbb{R}_{+}^{2}} w^{17} \exp \left(-\frac{1}{\lambda} w^{12}\right) \cdot t^{3} \exp \left(-\frac{1}{\lambda} t^{12}\right) d t d w
\end{aligned}
$$

(integrating by parts)

Let us remark that if we come back to $x$, we can write

$$
I_{1}=\int_{\mathbb{R}_{+}^{2}} x_{1}^{2} x_{2}^{4} \cdot \exp \left(-\frac{1}{\lambda}\left(x_{1}^{6}+x_{1}^{2} x_{2}^{2}\right)\right) d x
$$

which means that $12 I_{1}$ is the first term of the expansion of $L_{1}$ according to Lemma 4 .

However, it is simpler to set $w^{\prime}=w \lambda^{-1 / 12}$ and $t^{\prime}=t \lambda^{-1 / 12}$ thus

$$
\begin{aligned}
I_{1} & =\lambda^{\frac{18+4}{12}} \int_{\mathbb{R}_{+}^{2}} w^{17} \exp \left(-w^{12}\right) \cdot t^{\prime 3} \exp \left(-t^{\prime 12}\right) d t^{\prime} d w^{\prime} \\
& =\lambda^{11 / 6} \cdot \frac{1}{12^{2}} \Gamma\left(\frac{3}{2}\right) \Gamma\left(\frac{1}{3}\right)
\end{aligned}
$$

Study of $I_{2}$.

$$
I_{2}=-\int_{\mathbb{R}_{+}}\left[\sum_{s=1}^{12} c_{s}(\lambda, w)<\delta^{(s-1)} ; \phi_{\lambda}>\right] d w
$$

with $<\delta^{(s-1)} ; \phi_{\lambda}>=(-1)^{s-1} \frac{\partial^{s-1} \phi_{\lambda}}{\partial t^{s-1}}(w ; 0)$

so since $\phi_{\lambda}(w ; t)=w^{17} \exp \left(-\frac{1}{\lambda} w^{12}\right) t^{4} \exp \left(-\frac{1}{\lambda} t^{12}\right)$ we have

$$
\begin{gathered}
<\delta^{(s-1)} ; \phi_{\lambda}>=0 \text { if } s \in[1 ; 12] \text { and } s \neq 5 \\
<\delta^{(s-1)} ; \phi_{\lambda}>=24 . w^{17} \cdot \exp \left(-\frac{w^{12}}{\lambda}\right) \text { for } s=5 .
\end{gathered}
$$

This implies that

$$
I_{2}=-24 \int_{\mathbb{R}_{+}} c_{5}(\lambda, w) w^{17} \cdot \exp \left(-\frac{w^{12}}{\lambda}\right) d w
$$


where

$$
c_{5}(\lambda, w)=\lim _{t \rightarrow 0}\left(\left(f_{\lambda}\right)_{5 ; 5}(w ; t)+\frac{a_{4}}{24} \ln t\right)
$$

with $a_{4}=0$ and

$$
\left(f_{\lambda}\right)_{5 ; 5}(t)=\frac{(-1)^{5}}{24} \int_{t}^{\infty}(\tau-t)^{4}\left[\frac{1}{\tau} \exp \left(-\frac{1}{\lambda} \frac{w^{36}}{\tau^{12}}\right)-\frac{1}{\tau}\right] d \tau
$$

That leads to

$$
c_{5}(\lambda, w)=-\frac{1}{24} \int_{0}^{\infty} \tau^{4}\left[\frac{1}{\tau} \exp \left(-\frac{1}{\lambda} \frac{w^{36}}{\tau^{12}}\right)-\frac{1}{\tau}\right] d \tau
$$

and finally

$$
I_{2}=\int_{0}^{\infty} w^{17} \cdot \exp \left(-\frac{w^{12}}{\lambda}\right)\left(\int_{0}^{\infty} \tau^{3}\left[\exp \left(-\frac{1}{\lambda} \frac{w^{36}}{\tau^{12}}\right)-1\right] d \tau\right) d w
$$

and $u=w^{36} \lambda^{-1} \tau^{-12}$ gives

$$
I_{2}=\frac{1}{12} \lambda^{-1 / 3} \int_{0}^{\infty} w^{29} \cdot \exp \left(-\frac{w^{12}}{\lambda}\right) d w \cdot\left(\int_{0}^{\infty} u^{-4 / 3}[\exp (-u)-1] d u\right)
$$

and with $w^{\prime}=w \lambda^{-1 / 12}$

$$
\begin{aligned}
I_{2} & =-\lambda^{13 / 6} \int_{0}^{\infty} w^{\prime 29} \cdot \exp \left(-w^{12}\right) d w^{\prime} \cdot \int_{0}^{+\infty} u^{-4 / 3}(1-\exp (-u)) d u \\
& =-\lambda^{13 / 6} \cdot \frac{1}{12^{2}} \Gamma\left(\frac{5}{2}\right) \cdot \int_{0}^{+\infty} u^{-4 / 3}(1-\exp (-u)) d u
\end{aligned}
$$

The asymptotic expansion of $L_{1}$. Finally we find that $L_{1}=12\left(I_{1}+I_{2}\right)+o\left(\lambda^{5 / 2}\right)$ becomes

$$
\begin{aligned}
L_{1}= & \lambda^{11 / 6} \cdot \frac{1}{12} \Gamma\left(\frac{3}{2}\right) \Gamma\left(\frac{1}{3}\right) \\
& -\lambda^{13 / 6} \cdot \frac{1}{12} \Gamma\left(\frac{5}{2}\right) \int_{0}^{+\infty} u^{-4 / 3}(1-\exp (-u)) d u+o\left(\lambda^{5 / 2}\right) .
\end{aligned}
$$

\section{REFERENCES}

[1] V.I. ARnold, Singularities of Caustics and Wave Fronts, MIA, Kluwer (1990).

[2] V.I. Arnold, A. Varchenko and S. Husein-Sade, Singularities of differentiable maps II, Birkhauser (1988).

[3] E. Combet, Intégrales exponentielles, Lecture Notes in Mathematics 937, Springer-Verlag (1982).

[4] J. Denef and P. Sargos, Polyèdre de Newton et distribution $f_{+}^{s}$, I J. d'Anal.Math., 53, pp. 201-218.

[5] M. Dostal and B. Gaveau, The stationary phase method for certain degenerate critical points, I. Canad. J. of Math., (1989), pp. 907-931.

[6] A. ERdelyi, Asymptotic expansions, Dover (1956). 
[7] H. Hinonaka, Resolution of singularities of an algebraic variety over a field of characteristic zero, I.II. Ann. Math., 79 (1964), pp. 109-203, pp. 205-326.

[8] D. Kaminski And R. B. PARIS, Asymptotics of a class of multidimensional Laplace-type integrals, Phil. Trans. R. Soc. London. A, 356 (1998), pp. 583-667.

[9] Kouchnirenko, Polyèdres de Newton et nombres de Milnor, Inventiones mathematicae, 32 (1976), pp. 1-31.

[10] J.P. Mc CluRe And R. Wong, Explicit error terms for asymptotic expansions of Stieltjes transforms, J. Inst. Math. Applic., 22 (1978), pp. 129-145.

[11] S. Rossignol, Laplace's method on an abstract Wiener space, with degenerate minima, Preprint.

[12] A. VARChenKo, Newton Polyhedra and estimation of oscillating integrals, FAA, 10 (1976), pp. $175-196$.

[13] Vasil'Ev, Asymptotic exponential integrals, Newton's diagram, and the classification of minimal points, FAA, 11 (1977), pp. 163-172.

[14] R. Wong, Asymptotic approximations of integrals, Academic Press (1989). 